\title{
Statistical inference of subcritical strongly stationary Galton-Watson processes with regularly varying immigration
}

\author{
Mátyás Barczy ${ }^{* \diamond}$, Bojan BasraK**, Péter Kever***, \\ Gyula PaP ${ }^{* * * * * * *}$, Hrvoje Planinić ${ }^{* *}$
}

\begin{abstract}
* MTA-SZTE Analysis and Stochastics Research Group, Bolyai Institute, University of Szeged, Aradi vértanúk tere 1, 6720 Szeged, Hungary.

** Department of Mathematics, Faculty of Science, University of Zagreb, Bijenička 30, 10000 Zagreb, Croatia

*** Bolyai Institute, University of Szeged, Aradi vértanúk tere 1, 6720 Szeged, Hungary.

**** Alfréd Rényi Institute of Mathematics, Reáltanoda u. 13-15, 1053 Budapest, Hungary.

e-mails: barczy@math.u-szeged.hu (M. Barczy), bbasrak@math.hr (B. Basrak), kevei@math.uszeged.hu (P. Kevei), hrvoje.planinic@math.hr (H. Planinić).

$\diamond$ Corresponding author.
\end{abstract}

\begin{abstract}
We describe the asymptotic behavior of the conditional least squares estimator of the offspring mean for subcritical strongly stationary Galton-Watson processes with regularly varying immigration with tail index $\alpha \in(1,2)$. The limit law is the ratio of two dependent stable random variables with indices $\alpha / 2$ and $2 \alpha / 3$, respectively, and it has a continuously differentiable density function. We use point process technique in the proofs.
\end{abstract}

\section{Introduction}

The theory and estimation of branching processes, especially Galton-Watson processes without or with immigration has a long history, see, e.g., the survey paper by Winnicki [43]. In this paper we will consider a Galton-Watson process with regularly varying immigration distribution, and we will study the Conditional Least Squares (CLS) estimation of the mean of

2010 Mathematics Subject Classifications: 60J80, 62F12, 60G55.

Key words and phrases: Galton-Watson process with immigration, conditional least squares estimator, regularly varying distribution, strong stationarity, point process.

Supported by the Hungarian Croatian Intergovernmental S\&T Cooperation Programme for 2017-2018 under Grant No. 16-1-2016-0027. Mátyás Barczy and Péter Kevei are supported by the János Bolyai Research Scholarship of the Hungarian Academy of Sciences. Bojan Basrak, Péter Kevei and Hrvoje Planinić are financed within the Croatian-Swiss Research Program of the Croatian Science Foundation and the Swiss National Science Foundation - grant CSRP 018-01-180549. Péter Kevei and Gyula Pap are supported by the Ministry for Innovation and Technology, Hungary grant TUDFO/47138-1/2019-ITM, and by the EU-funded Hungarian grant EFOP-3.6.1-16-2016-00008. Péter Kevei is supported by the NKFIH grant FK124141. 
the offspring distribution. Heavy-tailed Galton-Watson processes with immigration, especially with regularly varying immigration distribution, have been in the focus of research for a long time. We name only two papers here. Seneta [40] derived conditions under which there exists a sequence of positive constants such that the logarithm of a Galton-Watson process having offspring distribution with infinite mean and normalized by the sequence in question converges in distribution to a non-degenerate distribution. Schuh and Barbour [39] derived necessary and sufficient conditions for the almost sure convergence of some slowly varying function of a Galton-Watson process having offspring distribution with infinite mean. Heavy-tailed branching processes are important not only from the theoretical point of view, but they are also used in modeling of biological phenomena, e.g., for modeling the development of multi-focal tumors, see Ernst et al. [15]. Concerning the estimation theory of heavy-tailed Galton-Watson processes with immigration we are not aware of any results. Let us recall now a result on the estimation of the offspring mean under finite third order moment assumptions.

Let $\mathbb{Z}$ and $\mathbb{N}$ denote the set of integers and positive integers, respectively. Every random variable will be defined on a fixed probability space $(\Omega, \mathcal{A}, \mathbb{P})$. For each $i, j \in \mathbb{N}$, the number of individuals in the $i^{\text {th }}$ generation will be denoted by $X_{i}$, the number of offspring produced by the $j^{\text {th }}$ individual belonging to the $(i-1)^{\text {th }}$ generation will be denoted by $A_{j}^{(i)}$, and the number of immigrants in the $i^{\text {th }}$ generation will be denoted by $B_{i}$. Further, $X_{0}$ denotes the size of the initial population. Then we have

$$
X_{i}=\sum_{j=1}^{X_{i-1}} A_{j}^{(i)}+B_{i}, \quad i \in \mathbb{N}
$$

where $\left\{A, A_{j}^{(i)}: i, j \in \mathbb{N}\right\}$ are independent, identically distributed (i.i.d.) nonnegative integervalued random variables independent of another i.i.d. sequence $\left\{B, B_{i}: i \in \mathbb{N}\right\}$ of nonnegative integer-valued random variables. Assuming that $X_{0}$ is independent of $\left\{A_{j}^{(i)}, B_{i}: i, j \in \mathbb{N}\right\}$, $\mathbb{E}\left[X_{0}\right]<\infty, \mu_{A}:=\mathbb{E}[A]<\infty, \mu_{B}:=\mathbb{E}[B] \in(0, \infty)$ and that $\mu_{B}$ is known, the CLS estimator of $\mu_{A}$ based on the observations $X_{0}, X_{1}, \ldots, X_{n}$ has the form

$$
{\widehat{\mu_{A}}}^{(n)}:=\frac{\sum_{i=1}^{n} X_{i-1}\left(X_{i}-\mu_{B}\right)}{\sum_{i=1}^{n} X_{i-1}^{2}}
$$

on the set $\left\{\sum_{i=1}^{n} X_{i-1}^{2}>0\right\}$, see Klimko and Nelson [24]. We have $\mathbb{P}\left(\sum_{i=1}^{n} X_{i-1}^{2}>0\right) \rightarrow 1$ as $n \rightarrow \infty$, since $\mathbb{P}\left(\sum_{i=1}^{n} X_{i-1}^{2}=0\right)=\mathbb{P}\left(X_{0}=0, B_{1}=0, \ldots, B_{n-1}=0\right) \leq \mathbb{P}(B=0)^{n-1} \rightarrow 0$ as $n \rightarrow \infty$ due to $\mathbb{P}(B=0) \in[0,1)$. If, in addition, $\mu_{A} \in(0,1)$, then the Markov chain $\left(X_{i}\right)_{i \geq 0}$ admits a unique stationary distribution, see, e.g., Quine [34]. If, in addition, $\mathbb{E}\left[X_{0}^{3}\right]<\infty$, $\mathbb{E}\left[A^{3}\right]<\infty$ and $\mathbb{E}\left[B^{3}\right]<\infty$, then

$$
n^{1 / 2}\left({\widehat{\mu_{A}}}^{(n)}-\mu_{A}\right) \stackrel{d}{\rightarrow} \mathcal{N}\left(0, \frac{\sigma_{A}^{2} \mathbb{E}\left[\widetilde{X}^{3}\right]+\sigma_{B}^{2} \mathbb{E}\left[\widetilde{X}^{2}\right]}{\left(\mathbb{E}\left[\widetilde{X}^{2}\right]\right)^{2}}\right) \quad \text { as } n \rightarrow \infty
$$

where $\sigma_{A}^{2}:=\operatorname{Var}(A)$ and $\sigma_{B}^{2}:=\operatorname{Var}(B)$, the distribution of the random variable $\tilde{X}$ is the unique stationary distribution of $\left(X_{i}\right)_{i \geq 0}$, and $\stackrel{d}{\longrightarrow}$ denotes convergence in distribution. The paper by 
Klimko and Nelson [24, Section 5] contains a similar result for the CLS estimator of $\left(\mu_{A}, \mu_{B}\right)$, and (1.2) can be derived by the method of that paper. Note that $\mathbb{E}\left[\tilde{X}^{2}\right]$ and $\mathbb{E}\left[\tilde{X}^{3}\right]$ can be expressed in terms of the first three moments of $A$ and $B$, see, e.g., Quine [34, formula (26) and page 422] and Barczy et al. [2, formulae (14), (16) and (20)].

In contrast with those earlier results, we explore the case where the distribution of $B$ is regularly varying with tail index in $(1,2)$, thus having infinite variance. In the sequel, we will always assume the following conditions:

(i) $\mu_{A} \in(0,1)$,

(ii) $\sigma_{A}^{2} \in(0, \infty)$,

(iii) $B$ is regularly varying with tail index $\alpha \in(1,2)$, i.e.,

$$
\lim _{x \rightarrow \infty} \frac{\mathbb{P}(B>q x)}{\mathbb{P}(B>x)}=q^{-\alpha} \quad \text { for all } q \in(0, \infty) .
$$

In particular, $\mu_{B} \in(0, \infty)$, and there exists a strongly stationary process $\left(X_{i}\right)_{i \in \mathbb{Z}}$ satisfying

$$
X_{i}=\sum_{j=1}^{X_{i-1}} A_{j}^{(i)}+B_{i}, \quad i \in \mathbb{Z},
$$

where $\left\{A, A_{j}^{(i)}: j \in \mathbb{N}, i \in \mathbb{Z}\right\}$ are i.i.d. nonnegative integer-valued random variables independent of another i.i.d. sequence $\left\{B, B_{i}: i \in \mathbb{Z}\right\}$ of nonnegative integer-valued random variables. Note that, for simplicity, in (1.3) we keep the same letter $X$ to denote the strongly stationary extension of the process indexed by $\mathbb{Z}$. In this case, the distribution of $X_{0}$ is also regularly varying with the same tail index $\alpha$ having infinite variance, or more precisely,

$$
\mathbb{P}\left(X_{0}>x\right) \sim \frac{1}{1-\mu_{A}^{\alpha}} \mathbb{P}(B>x) \quad \text { as } x \rightarrow \infty,
$$

see Basrak et al. [5, Theorem 2.1.1].

Our aim is to study the limiting behavior of ${\widehat{\mu_{A}}}^{(n)}$ as $n \rightarrow \infty$ for the strongly stationary process $\left(X_{i}\right)_{i \in \mathbb{Z}}$ given in (1.3). For each $n \in \mathbb{N}$, by (1.1), we have

$$
{\widehat{\mu_{A}}}^{(n)}-\mu_{A}=\frac{\sum_{i=1}^{n} X_{i-1}\left(X_{i}-\mu_{B}\right)}{\sum_{i=1}^{n} X_{i-1}^{2}}-\mu_{A}=\frac{\sum_{i=1}^{n} X_{i-1} M_{i}}{\sum_{i=1}^{n} X_{i-1}^{2}}
$$

on the set $\left\{\sum_{i=1}^{n} X_{i-1}^{2}>0\right\}$, where, by (1.3),

$$
M_{i}:=X_{i}-\mu_{A} X_{i-1}-\mu_{B}=\sum_{j=1}^{X_{i-1}}\left(A_{j}^{(i)}-\mu_{A}\right)+\left(B_{i}-\mu_{B}\right)=: \sum_{j=1}^{X_{i-1}} \tilde{A}_{j}^{(i)}+\tilde{B}_{i}, \quad i \in \mathbb{Z} .
$$

Intuitively, by the central limit theorem, for large $X_{i-1}$, the distribution of $M_{i} / \sqrt{X_{i-1}}$ is approximately normal, thus, in the spirit of Breiman's lemma, $M_{i} X_{i-1}=X_{i-1}^{3 / 2} M_{i} / \sqrt{X_{i-1}}$ is regularly varying with tail index $2 \alpha / 3$. This argument is made precise in Proposition 2.1. 
Our analysis relies on the fact that one can determine the weak limit of the point processes

$$
\sum_{j=1}^{n} \delta_{\left(\frac{X_{j}}{a_{n}}, \frac{M_{j+1}}{\sqrt{X_{j}}}\right)}:=\sum_{\left\{j \in\{1, \ldots, n\}: X_{j}>0\right\}} \delta\left(\frac{X_{j}}{a_{n}}, \frac{M_{j+1}}{\sqrt{X_{j}}}\right)
$$

as $n \rightarrow \infty$ on a suitable space of point measures on $(0, \infty) \times \mathbb{R}$ and with a scaling sequence $\left(a_{n}\right)_{n \in \mathbb{N}}$ satisfying $n \mathbb{P}\left(X_{0}>a_{n}\right) \rightarrow 1$ as $n \rightarrow \infty$ (see (3.1)), where $\delta_{(x, y)}$ denotes the Dirac measure concentrated on $(x, y) \in(0, \infty) \times \mathbb{R}$, see Theorem 3.2. For a possible choice of a suitable sequence $\left(a_{n}\right)_{n \in \mathbb{N}}$ and its asymptotic behavior as $n \rightarrow \infty$, see the beginning of Section 3 . The proof of Theorem 3.2 is based on general results of Kallenberg [21, Theorems 4.11 and 4.22] for convergence in distribution of random measures with respect to the vague topology. Point processes have been often applied to analyze regularly varying observations, see, for instance, Resnick [35, 36] and Kulik and Soulier [27], however, our approach here is not standard since we topologize the space of point measures on $(0, \infty) \times \mathbb{R}$ using vague topology with "bounded" Borel sets being those which are bounded away from the vertical line $\{0\} \times \mathbb{R}$ instead of being bounded away from the point $(0,0)$. For a detailed discussion on our setup, see the beginning of Section 3 and Appendix A. In the course of the proof of Theorem 3.2, the joint tail behavior of $\left(X_{i}\right)_{i \in \mathbb{Z}}$ and $\left(M_{i+1} / \sqrt{\max \left(X_{i}, 1\right)}\right)_{i \in \mathbb{Z}_{+}}$, especially the so-called tail process of $\left(X_{i}\right)_{i \in \mathbb{Z}}$, also plays a crucial role, see Proposition 2.1 and (2.1).

Based on convergence of the point processes in (1.6), we obtain

$$
\sqrt{a_{n}}\left({\widehat{\mu_{A}}}^{(n)}-\mu_{A}\right) \stackrel{d}{\longrightarrow} \frac{V^{(2)}}{V^{(1)}} \quad \text { as } n \rightarrow \infty,
$$

where $V^{(1)}$ is an $\alpha / 2$-stable positive random variable, $V^{(2)}$ is a symmetric $2 \alpha / 3$-stable random variable, and $V^{(1)}$ and $V^{(2)}$ are dependent with an explicitly given joint characteristic function, see Theorem 5.1. Concerning the asymptotic behavior of $\left(a_{n}\right)_{n \in \mathbb{N}}$, note that if $x^{\alpha} \mathbb{P}(B>x) \rightarrow 1$ as $x \rightarrow \infty$, i.e., the distribution of $B$ is asymptotically equivalent to a Pareto distribution with parameter $\alpha$, then $n^{-1 / \alpha} a_{n} \rightarrow\left(1-\mu_{A}^{\alpha}\right)^{-1 / \alpha}$ as $n \rightarrow \infty$. Indeed, using (1.4) and that $n \mathbb{P}\left(X_{0}>a_{n}\right) \rightarrow 1$ as $n \rightarrow \infty$, we have

$$
n^{-1 / \alpha} a_{n}=\left(n^{-1} a_{n}^{\alpha}\right)^{1 / \alpha} \sim\left(a_{n}^{\alpha} \mathbb{P}\left(X_{0}>a_{n}\right)\right)^{1 / \alpha} \sim\left(a_{n}^{\alpha}\left(1-\mu_{A}^{\alpha}\right)^{-1} \mathbb{P}\left(B>a_{n}\right)\right)^{1 / \alpha} \sim\left(1-\mu_{A}^{\alpha}\right)^{-1 / \alpha}
$$

as $n \rightarrow \infty$, as desired.

In Section 5, we collect several properties of $\left(V^{(1)}, V^{(2)}\right)$ and $V^{(2)} / V^{(1)}$, including that the distribution of $\left(V^{(1)}, V^{(2)}\right)$ is operator stable and $V^{(2)} / V^{(1)}$ has a continuously differentiable density function. In Appendix A, we collect some topological properties of $(0, \infty) \times \mathbb{R}$. Appendix $\mathrm{B}$ contains the proof of Lemma 3.1 describing vague convergence of point measures. In Appendix $\mathrm{C}$ we show that the process $\left(X_{i} \mathbb{1}_{\left\{X_{i}>0\right\}}, \frac{M_{i+1}}{\sqrt{X_{i}}} \mathbb{1}_{\left\{X_{i}>0\right\}}\right)_{i \geq 0}$ satisfies a certain mixing condition. Appendix D is devoted to a conditional Slutsky's lemma and a conditional continuous mapping theorem. In Appendix E, we show that the process $\left(X_{i}^{3 / 2}, X_{i} M_{i+1}\right)_{i \in \mathbb{Z}}$ is regularly varying with tail index $2 \alpha / 3$ with an explicitly given forward tail process.

We note that our proof technique does not work for the case $\alpha=2$. Formally, one can see that the fact $\alpha<2$ is used many times in the proofs, and, for example, in case $\alpha=2$ the series 
$\sum_{i=1}^{\infty} P_{i}^{2}$ appearing as the weak limit of $\left(1-\mu_{A}^{2}\right) a_{n}^{-2} \sum_{j=1}^{n} X_{j}^{2}$ as $n \rightarrow \infty$ in Theorem 4.1 is not convergent almost surely by Campbell's theorem (see, e.g., Kingman [23, Section 3.2]) due to $\int_{0}^{\infty}\left(y^{2} \wedge 1\right) \mathrm{d}\left(-y^{-2}\right)=\infty$. In case of $\alpha=2, X_{j}^{2}, j \in \mathbb{N}$, is positive almost surely and regularly varying with tail index 1 , so in order to get some weak limit of $\sum_{j=1}^{n} X_{j}^{2}$ as $n \rightarrow \infty$ one may need to introduce an appropriate centering as well.

Our results can be compared with the results on $\mathrm{AR}(1)$ processes

$$
\xi_{i}=\phi \xi_{i-1}+\epsilon_{i}, \quad i \in \mathbb{N},
$$

where $\phi \in \mathbb{R}$ and $\left\{\epsilon, \epsilon_{i}: i \in \mathbb{N}\right\}$ are i.i.d. random variables. Assuming that $\xi_{0}$ is independent of $\left\{\epsilon_{i}: i \in \mathbb{N}\right\}, \mathbb{E}[\epsilon]=0$, the CLS estimator of $\phi$ based on the observations $\xi_{0}, \xi_{1}, \ldots, \xi_{n}$ has the form

$$
\widehat{\phi}_{n}:=\frac{\sum_{i=1}^{n} \xi_{i-1} \xi_{i}}{\sum_{i=1}^{n} \xi_{i-1}^{2}}
$$

on the set $\left\{\sum_{i=1}^{n} \xi_{i-1}^{2}>0\right\}$. If, in addition, $\phi \in(-1,1)$, then the Markov chain $\left(\xi_{i}\right)_{i \geq 0}$ admits a unique stationary distribution, and there exists a strongly stationary process $\left(\xi_{i}\right)_{i \in \mathbb{Z}}$ satisfying

$$
\xi_{i}=\phi \xi_{i-1}+\epsilon_{i}, \quad i \in \mathbb{Z},
$$

where $\left\{\epsilon, \epsilon_{i}: i \in \mathbb{Z}\right\}$ are i.i.d. random variables. If, in addition, $\epsilon$ is symmetric and regularly varying with tail index $\alpha \in(0,2)$, then

$$
\widehat{\phi}_{n} \rightarrow \phi \quad \text { as } n \rightarrow \infty \text { almost surely, }
$$

see Hannan and Kanter [18], and

$$
b_{n}\left(\widehat{\phi}_{n}-\phi\right) \stackrel{d}{\longrightarrow} \frac{U^{(2)}}{U^{(1)}} \quad \text { as } n \rightarrow \infty
$$

under the additional assumption $\lim _{x \rightarrow \infty} \frac{\mathbb{P}\left(\left|\epsilon_{0} \epsilon_{1}\right|>x\right)}{\mathbb{P}\left(\left|\epsilon_{0}\right|>x\right)}=2 \mathbb{E}\left[|\epsilon|^{\alpha}\right]$ in case of $\mathbb{E}\left[|\epsilon|^{\alpha}\right]<\infty$, where $\left(b_{n}\right)_{n \in \mathbb{N}}$ is a suitable scaling sequence, $U^{(1)}$ is an $\alpha / 2$-stable positive random variable and $U^{(2)}$ is a symmetric $\alpha$-stable random variable, see Davis and Resnick [12, Theorem 3.6] for the case of $\mathbb{E}\left[|\epsilon|^{\alpha}\right]<\infty$ and Davis and Resnick [13, Theorem 4.4] for the case of $\mathbb{E}\left[|\epsilon|^{\alpha}\right]=\infty$. This representation of the limit distribution is derived in Davis and Resnick [13, Example 5]. Further, $U^{(1)}$ and $U^{(2)}$ are claimed to be dependent in case of $\mathbb{E}\left[|\epsilon|^{\alpha}\right]<\infty$, while they are independent in case of $\mathbb{E}\left[|\epsilon|^{\alpha}\right]=\infty$. In fact, if $\mathbb{E}\left[|\epsilon|^{\alpha}\right]<\infty$, then $b_{n}, n \geq 2$, is the $1-\frac{1}{n}$ lower quantile of $|\epsilon|$, and one can also write $b_{n}=n^{\frac{1}{\alpha}} L_{1}(n), n \in \mathbb{N}$, with some slowly varying function $L_{1}:(0, \infty) \rightarrow(0, \infty)$. If $\mathbb{E}\left[|\epsilon|^{\alpha}\right]=\infty$, then $b_{n}=\widetilde{c}_{n}^{-1} c_{n}^{2}, n \geq 2$, where $c_{n}$ is the $1-\frac{1}{n}$ lower quantile of $\left|\epsilon_{1}\right|$, and $\widetilde{c}_{n}$ is the $1-\frac{1}{n}$ lower quantile of $\left|\epsilon_{0} \epsilon_{1}\right|$, and in this case one can also write $b_{n}=n^{\frac{1}{\alpha}} L_{2}(n), n \in \mathbb{N}$, with some slowly varying function $L_{2}:(0, \infty) \rightarrow(0, \infty)$. Moreover, if $x^{\alpha} \mathbb{P}(|\epsilon|>x) \rightarrow 1$ as $x \rightarrow \infty$, i.e., the distribution of $|\epsilon|$ is asymptotically equivalent to a Pareto distribution with parameter $\alpha$, then $\mathbb{E}\left[|\epsilon|^{\alpha}\right]=\infty$ and $n^{-1 / \alpha}(\log (n))^{1 / \alpha} b_{n} \rightarrow 1$ as $n \rightarrow \infty$, see Resnick [36, Problem 9.13]. We also emphasize that Galton-Watson processes with immigration are quite different from $\mathrm{AR}(1)$ process due to the branching property of the 
process. As a consequence, for example, the unique stationary distribution of the process can be represented in a more complicated way compared to that of an $\mathrm{AR}(1)$ process, see Lemma E.2. So our point process technique for the proof is not a simple modification of the known one for $\operatorname{AR}(1)$ processes.

Finally, we recall two results on the CLS estimator of some parameters for related heavytailed continuous time processes.

$\mathrm{Hu}$ and Long [19] studied the asymptotic behavior of the least squares estimator of the drift parameter for a generalized Ornstein-Uhlenbeck process driven by a symmetric $\alpha$-stable Lévy motion with $\alpha \in(0,2)$ in the ergodic case based on discrete time infill-increasing (high frequency) observations. Using some results of Davis and Resnick [13], Hu and Long [19] proved strong consistency of the least squares estimator in question, and they also described its asymptotic behavior with a limit distribution being the fraction of two independent stable random variables.

Li and Ma [29], using a similar point process technique, described somewhat similar asymptotic behavior of the weighted and non-weighted CLS estimators of the drift parameters for a stable Cox-Ingersoll-Ross model based on low frequency observations. This process can be viewed as a special subcritical continuous state and continuous time branching process with immigration.

Convergence in probability and in $L_{2}$ will be denoted by $\stackrel{\mathbb{P}}{\longrightarrow}$ and $\stackrel{L_{2}}{\longrightarrow}$, respectively. Weak convergence of finite measures will be denoted by $\stackrel{w}{\longrightarrow}$, and convergence of finite dimensional distributions is denoted by $\stackrel{\text { fi.di. }}{\longrightarrow}$. The diagonal matrix with diagonal entries $a_{1}, \ldots, a_{n} \in \mathbb{R}$ is denoted by $\operatorname{diag}_{n}\left(a_{1}, \ldots, a_{n}\right)$. We write $\delta_{h}$ for the Dirac measure on a set $H$ concentrated at $h \in H$. For a random vector $\boldsymbol{X}$ and event $A \in \mathcal{A}$ such that $\mathbb{P}(A)>0, \mathcal{L}(\boldsymbol{X})$ and $\mathcal{L}(\boldsymbol{X} \mid A)$ denote the law of $\boldsymbol{X}$ and law of $\boldsymbol{X}$ conditionally on $A$, respectively.

\section{Tail behavior of the process}

In what follows, let us consider the strongly stationary process $\left(X_{i}\right)_{i \in \mathbb{Z}}$ given in (1.3). For determining the weak limit of the point processes given in (1.6), in the proof of Theorem 3.2 the joint tail behavior of $\left(X_{i}\right)_{i \in \mathbb{Z}}$ and $\left(M_{i+1} / \sqrt{\max \left(X_{i}, 1\right)}\right)_{i \in \mathbb{Z}_{+}}$, especially the so-called tail process of $\left(X_{i}\right)_{i \in \mathbb{Z}}$, plays a crucial role. First, we present the tail process of $\left(X_{i}\right)_{i \in \mathbb{Z}}$, and then we formulate a result on the tail behavior of the above mentioned two stochastic processes.

The process $\left(X_{i}\right)_{i \in \mathbb{Z}}$ is jointly regularly varying with tail index $\alpha$ and admits a tail process $\left(Y_{i}\right)_{i \in \mathbb{Z}}$, i.e. all the finite dimensional distributions of $\left(X_{i}\right)_{i \in \mathbb{Z}}$ are regularly varying with tail index $\alpha$, and for all $m \in \mathbb{N}$,

$$
\mathcal{L}\left(x^{-1} X_{-m}, \ldots, x^{-1} X_{m} \mid X_{0}>x\right) \stackrel{w}{\longrightarrow} \mathcal{L}\left(Y_{-m}, \ldots, Y_{m}\right),
$$

as $x \rightarrow \infty$. Indeed, Basrak et al. [5, Lemma 3.1] showed the existence of the forward tail process $\left(Y_{i}\right)_{i \geq 0}$ of the sequence $\left(X_{i}\right)_{i \in \mathbb{Z}}$, and, by Theorem 2.1 in Basrak and Segers [7], the existence 
of the forward tail process is equivalent to the existence of the (whole) tail process and to the joint regular variation of $\left(X_{i}\right)_{i \in \mathbb{Z}}$ as well. We claim that

$$
Y_{-K+i}= \begin{cases}\mu_{A}^{-K+i} Y_{0}, & i \geq 0 \\ 0, & i<0\end{cases}
$$

yielding

$$
Y_{i}= \begin{cases}\mu_{A}^{i} Y_{0}, & i \geq 0, \\ \mu_{A}^{i} \mathbb{1}_{\{K \geq|i|\}} Y_{0}, & i<0,\end{cases}
$$

where $Y_{0}$ is a Pareto distributed random variable such that $\mathbb{P}\left(Y_{0} \geq y\right)=y^{-\alpha}$ for $y \geq 1$, and $K$ is a geometrically distributed random variable independent of $Y_{0}$ such that

$$
\mathbb{P}(K=k)=\mu_{A}^{\alpha k}\left(1-\mu_{A}^{\alpha}\right), \quad k=0,1,2, \ldots
$$

Indeed, as shown in Basrak et al. [5, Lemma 3.1], $\left(Y_{i}\right)_{i \geq 0}$ is the forward tail process of the sequence $\left(X_{i}\right)_{i \in \mathbb{Z}}$. On the other hand, by Janssen and Segers [20, Example 6.2], $\left(Y_{i}\right)_{i \in \mathbb{Z}}$ is the tail process of the stationary solution $\left(\tilde{\xi}_{i}\right)_{i \in \mathbb{Z}}$ to the stochastic recurrence equation $\tilde{\xi}_{i}=\mu_{A} \tilde{\xi}_{i-1}+B_{i}$, $i \in \mathbb{Z}$. Since the distribution of the forward tail process determines the distribution of the (whole) tail process (see Basrak and Segers [7, Theorem 2.1]), it follows that $\left(Y_{i}\right)_{i \in \mathbb{Z}}$ represents the tail process of $\left(X_{i}\right)_{i \in \mathbb{Z}}$.

For the ease of notation, denote

$$
W_{i}:=\frac{M_{i+1}}{\sqrt{\mu_{A}^{i} X_{0}}}, \quad W_{i}^{\prime}:=\frac{M_{i+1}}{\sqrt{X_{i} \vee 1}}, \quad i \geq 0,
$$

on the set $\left\{X_{0}>0\right\}$, where $a \vee b:=\max \{a, b\}, a, b \in \mathbb{R}$. Note that $\mathbb{P}\left(X_{0}=0\right)>0$ might occur. For example, if $\mathbb{P}(B \geq k)=c k^{-\alpha}$ for $k \in \mathbb{N}$ and $\mathbb{P}(B=0)=1-c$ with some $c \in(0,1)$, then $\mu_{B}=c \sum_{k=1}^{\infty} k^{-\alpha}$, hence in case of $c<\frac{1-\mu_{A}}{\sum_{k=1}^{\infty} k^{-\alpha}}$, we have $\mathbb{E}\left(X_{0}\right)=\frac{\mu_{B}}{1-\mu_{A}}<1$, yielding $\mathbb{P}\left(X_{0}=0\right)>0$, since $X_{0}$ is a nonnegative integer valued random variable.

Proposition 2.1. As $x \rightarrow \infty$,

$$
\mathcal{L}\left(x^{-1} X_{-m}, \ldots, x^{-1} X_{m}, W_{0}^{\prime}, \ldots, W_{m}^{\prime} \mid X_{0}>x\right) \stackrel{w}{\longrightarrow} \mathcal{L}\left(Y_{-m}, \ldots, Y_{m}, Z_{0}, \ldots, Z_{m}\right)
$$

for all $m \in \mathbb{N}$, where $\left(Z_{i}\right)_{i \geq 0}$ is an i.i.d. sequence of $\mathcal{N}\left(0, \sigma_{A}^{2}\right)$-distributed random variables being independent of $Y_{0}$ and $K$ with $\sigma_{A}^{2}=\operatorname{Var}(A) \in(0, \infty)$.

Although one can prove that the two-dimensional process $\left(X_{i}^{3 / 2}, X_{i} M_{i+1}\right)_{i \in \mathbb{Z}}$ admits a tail process in the sense of Basrak and Segers [7], see Appendix E, and then use standard point process convergence results for describing the asymptotic behavior of ${\widehat{\mu_{A}}}^{(n)}$ as $n \rightarrow \infty$, such an approach turns out to be rather complicated. However, for the purpose of our analysis, the statement of Proposition 2.1 turns out to be sufficient. Note that this approach is similar to the so-called conditional extreme value approach, see Kulik and Soulier [26] and references therein. 
Proof of Proposition 2.1. Let $m \in \mathbb{N}$ be fixed. First, note that $Y_{0}^{-1} Y_{i}=\mu_{A}^{i}, i \geq 0$, is the forward spectral process of $\left(X_{k}\right)_{k \in \mathbb{Z}}$, so by part (ii) of Corollary 3.2 in Basrak and Segers [7],

$$
\mathcal{L}\left(X_{0}^{-1} X_{0}, \ldots, X_{0}^{-1} X_{m} \mid X_{0}>x\right) \stackrel{w}{\longrightarrow} \delta_{\left(1, \mu_{A}, \ldots, \mu_{A}^{m}\right)} \quad \text { as } x \rightarrow \infty .
$$

In particular, for every $i \geq 0$ and $\epsilon>0$, since convergence in distribution to a constant implies convergence in probability (formally applying part (ii) of Lemma D.3 with the Borel measurable function $h: \mathbb{R} \rightarrow \mathbb{R}, h(x):=\mathbf{1}_{\left\{\left|\mu_{A}^{-i} x-1\right|>\epsilon\right\}}, x \in \mathbb{R}$, satisfying $D_{h}=\left\{\mu_{A}^{i}(1 \pm \epsilon)\right\}$ and $\mathbb{P}\left(\mu_{A}^{i} \in D_{h}\right)=0$ with $D_{h}$ being the set of discontinuities of $\left.h\right)$, we have

$$
\lim _{x \rightarrow \infty} \mathbb{P}\left(\left|\left(\mu_{A}^{i} X_{0}\right)^{-1} X_{i}-1\right|>\epsilon \mid X_{0}>x\right)=0 .
$$

Moreover, it is enough to show $(2.2)$ for $W_{0}, \ldots, W_{m}$ instead of $W_{0}^{\prime}, \ldots, W_{m}^{\prime}$. Indeed, one can easily check that

$$
\mathcal{L}\left(X_{0}^{-1} \mid X_{0}>x\right) \stackrel{w}{\longrightarrow} \delta_{0} \quad \text { as } x \rightarrow \infty,
$$

hence, by (2.3) and Lemma D.4, we obtain

$$
\mathcal{L}\left(X_{0}^{-1}, X_{0}^{-1} X_{0}, \ldots, X_{0}^{-1} X_{m} \mid X_{0}>x\right) \stackrel{w}{\longrightarrow} \delta_{\left(0,1, \mu_{A}, \ldots, \mu_{A}^{m}\right)} \quad \text { as } x \rightarrow \infty .
$$

Then, identifying $\mathbb{R}^{(3 m+2) \times(3 m+2)}$ with $\mathbb{R}^{(3 m+2)^{2}}$ in a natural way, we can use a conditional version of the continuous mapping theorem (see part (i) of Lemma D.3), and we get

$$
\mathcal{L}\left(\operatorname{diag}_{3 m+2}\left(1, \ldots, 1, \sqrt{\frac{X_{0}}{X_{0} \vee 1}}, \ldots, \sqrt{\frac{\mu_{A}^{m} X_{0}}{X_{m} \vee 1}}\right) \mid X_{0}>x\right) \stackrel{w}{\longrightarrow} \delta_{\operatorname{diag}_{3 m+2}(1, \ldots, 1)}
$$

as $x \rightarrow \infty$. Consequently, (2.2) with $W_{i}^{\prime}$ replaced by $W_{i}$ and Lemma D.5 imply (2.2).

Define now for $i \geq 0$ and $n \geq 1$,

$$
W_{i}^{\prime \prime}(n):=\left(\mu_{A}^{i} n\right)^{-1 / 2}\left(\sum_{j=1}^{\left\lfloor\mu_{A}^{i} n\right\rfloor} \tilde{A}_{j}^{(i+1)}+\tilde{B}_{i+1}\right)
$$

where $\tilde{A}_{j}^{(i+1)}$ and $\tilde{B}_{i+1}$ are introduced in (1.5). Take arbitrary $y \geq 1, u_{-m}, \ldots, u_{m} \in \mathbb{R}$ and introduce the events

$$
\begin{array}{ll}
C(x):=\left\{X_{-m} \leq x u_{-m}, \ldots, X_{-1} \leq x u_{-1}\right\}, & x \in(0, \infty), \\
D(n):=\left\{W_{0}^{\prime \prime}(n) \leq u_{0}, \ldots, W_{m}^{\prime \prime}(n) \leq u_{m}\right\}, & n \in \mathbb{N} .
\end{array}
$$

Observe that the definition of the tail process $\left(Y_{j}\right)_{j \in \mathbb{Z}}$ of $\left(X_{j}\right)_{j \in \mathbb{Z}}$ implies

$$
\lim _{x \rightarrow \infty} \mathbb{P}\left(X_{0}>x y, C(x) \mid X_{0}>x\right)=\mathbb{P}\left(Y_{0}>y, Y_{-m} \leq u_{-m}, \ldots, Y_{-1} \leq u_{-1}\right) .
$$

Further, the central limit theorem, Slutsky's lemma and independence of $W_{0}^{\prime \prime}(n), \ldots, W_{m}^{\prime \prime}(n)$ imply that

$$
\begin{aligned}
\lim _{n \rightarrow \infty} \mathbb{P}(D(n)) & =\lim _{n \rightarrow \infty}\left(\mathbb{P}\left(W_{0}^{\prime \prime}(n) \leq u_{0}\right) \cdots \mathbb{P}\left(W_{m}^{\prime \prime}(n) \leq u_{m}\right)\right) \\
& =\mathbb{P}\left(Z_{0} \leq u_{0}\right) \cdots \mathbb{P}\left(Z_{m} \leq u_{m}\right)=\mathbb{P}\left(Z_{0} \leq u_{0}, \ldots, Z_{m} \leq u_{m}\right),
\end{aligned}
$$


where, recall, $Z_{i}, i \geq 0$, are independent and $\mathcal{N}\left(0, \sigma_{A}^{2}\right)$-distributed random variables. Since $\left(\tilde{A}_{j}^{(i+1)}, \tilde{B}_{i+1}: i \geq 0, j \geq 1\right)$ are independent of $X_{-m}, \ldots, X_{0},(2.5)$ and (2.6) imply that for every $\epsilon>0$,

$$
\begin{aligned}
& \limsup _{x \rightarrow \infty} \mathbb{P}\left(X_{0}>x y, C(x), D\left(X_{0}\right) \mid X_{0}>x\right) \\
& =\limsup _{x \rightarrow \infty} \mathbb{P}\left(X_{0}>x\right)^{-1} \sum_{n>x y, n \in \mathbb{N}} \mathbb{P}\left(X_{0}=n, C(x)\right) \mathbb{P}(D(n)) \\
& \leq \limsup _{x \rightarrow \infty} \mathbb{P}\left(X_{0}>x\right)^{-1} \sum_{n>x y, n \in \mathbb{N}} \mathbb{P}\left(X_{0}=n, C(x)\right)\left(\mathbb{P}\left(Z_{0} \leq u_{0}, \ldots, Z_{m} \leq u_{m}\right)+\epsilon\right) \\
& =\left(\mathbb{P}\left(Z_{0} \leq u_{0}, \ldots, Z_{m} \leq u_{m}\right)+\epsilon\right) \limsup _{x \rightarrow \infty} \mathbb{P}\left(X_{0}>x y, C(x) \mid X_{0}>x\right) \\
& =\left(\mathbb{P}\left(Z_{0} \leq u_{0}, \ldots, Z_{m} \leq u_{m}\right)+\epsilon\right) \mathbb{P}\left(Y_{0}>y, Y_{-m} \leq u_{-m}, \ldots, Y_{-1} \leq u_{-1}\right) .
\end{aligned}
$$

An analogous claim holds for the limit inferior, and now letting $\epsilon \rightarrow 0$ yields that

$$
\begin{aligned}
\lim _{x \rightarrow \infty} \mathbb{P}\left(X_{0}>x y, C(x), D\left(X_{0}\right) \mid X_{0}>x\right) & \\
\quad= & \mathbb{P}\left(Y_{0}>y, Y_{-m} \leq u_{-m}, \ldots, Y_{-1} \leq u_{-1}, Z_{0} \leq u_{0}, \ldots, Z_{m} \leq u_{m}\right),
\end{aligned}
$$

since $\left(Z_{j}\right)_{j \geq 0}$ is assumed to be independent of $\left(Y_{j}\right)_{j \in \mathbb{Z}}$, hence

$$
\begin{aligned}
\mathcal{L}\left(x^{-1} X_{-m}, \ldots, x^{-1} X_{0}, W_{0}^{\prime \prime}\left(X_{0}\right), \ldots, W_{m}^{\prime \prime}\left(X_{0}\right)\right. & \left.\mid X_{0}>x\right) \\
& \stackrel{w}{\longrightarrow} \mathcal{L}\left(Y_{-m}, \ldots, Y_{0}, Z_{0}, \ldots, Z_{m}\right) \quad \text { as } x \rightarrow \infty .
\end{aligned}
$$

Using (2.3) and part (ii) of Lemma D.4, we get

$$
\begin{aligned}
\mathcal{L}\left(x^{-1} X_{-m}, \ldots, x^{-1} X_{0}, X_{0}^{-1} X_{1}, \ldots,\right. & \left.X_{0}^{-1} X_{m}, W_{0}^{\prime \prime}\left(X_{0}\right), \ldots, W_{m}^{\prime \prime}\left(X_{0}\right) \mid X_{0}>x\right) \\
& \stackrel{w}{\longrightarrow} \mathcal{L}\left(Y_{-m}, \ldots, Y_{0}, \mu_{A}, \ldots, \mu_{A}^{m}, Z_{0}, \ldots, Z_{m}\right) \quad \text { as } x \rightarrow \infty .
\end{aligned}
$$

Hence, using $x^{-1} X_{i}=\left(X_{0}^{-1} X_{i}\right)\left(x^{-1} X_{0}\right)$ and $Y_{i}=\mu_{A}^{i} Y_{0}$ for $i=1, \ldots, m$, together with Lemma D.3, we obtain

$$
\begin{aligned}
\mathcal{L}\left(x^{-1} X_{-m}, \ldots, x^{-1} X_{m}, W_{0}^{\prime \prime}\left(X_{0}\right), \ldots,\right. & \left.W_{m}^{\prime \prime}\left(X_{0}\right) \mid X_{0}>x\right) \\
& \stackrel{w}{\longrightarrow} \mathcal{L}\left(Y_{-m}, \ldots, Y_{m}, Z_{0}, \ldots, Z_{m}\right) \quad \text { as } x \rightarrow \infty .
\end{aligned}
$$

Next, we show that the above convergence holds with $W_{i}^{\prime \prime}\left(X_{0}\right)$ replaced by $W_{i}, i=1, \ldots, m$, which yields (2.2) (as explained at the beginning of the proof). For this purpose, we prove that

$$
\mathcal{L}\left(\Delta_{0}, \ldots, \Delta_{m} \mid X_{0}>x\right) \stackrel{w}{\longrightarrow} \delta_{(0, \ldots, 0)} \quad \text { as } x \rightarrow \infty,
$$


where, for $i \geq 0$,

$$
\Delta_{i}:=W_{i}-W_{i}^{\prime \prime}\left(X_{0}\right)=\frac{1}{\sqrt{\mu_{A}^{i} X_{0}}}\left(\sum_{j=1}^{X_{i}} \tilde{A}_{j}^{(i+1)}-\sum_{j=1}^{\left\lfloor\mu_{A}^{i} X_{0}\right\rfloor} \tilde{A}_{j}^{(i+1)}\right)
$$

on the set $\left\{X_{0}>0\right\}$. Note that $\Delta_{0}=0$. It is enough to check that for all $\epsilon \in(0,1)$,

$$
\limsup _{x \rightarrow \infty} \mathbb{P}\left(\left|\Delta_{i}\right|>\epsilon \mid X_{0}>x\right) \leq 2 \epsilon \sigma_{A}^{2}, \quad i=0, \ldots, m,
$$

since then, by part (i) of Lemma D.4, (2.8) follows. We will adapt the proof of Rényi's version of the Anscombe's theorem in Gut [17, page 347]. For proving (2.9), let $i \in\{0, \ldots, m\}$ be fixed. For all $\epsilon \in(0,1)$ and $\ell \in \mathbb{N}$, let us introduce the notations

$$
n_{0}(\ell):=\left\lfloor\mu_{A}^{i} \ell\right\rfloor, \quad n_{1}(\ell):=\left\lfloor\left(1-\epsilon^{3}\right) \mu_{A}^{i} \ell\right\rfloor+1, \quad n_{2}(\ell):=\left\lfloor\left(1+\epsilon^{3}\right) \mu_{A}^{i} \ell\right\rfloor .
$$

For all $\epsilon \in(0,1)$ and $k \in \mathbb{N}$, with the notation $S_{k}:=\sum_{j=1}^{k} \tilde{A}_{j}^{(i+1)}$, we have

$$
\begin{aligned}
\mathbb{P}\left(\left|\Delta_{i}\right|>\epsilon \mid X_{0}>x\right)= & \mathbb{P}\left(\left|S_{X_{i}}-S_{n_{0}\left(X_{0}\right)}\right|>\epsilon \sqrt{\mu_{A}^{i} X_{0}} \mid X_{0}>x\right) \\
= & \mathbb{P}\left(\left|S_{X_{i}}-S_{n_{0}\left(X_{0}\right)}\right|>\epsilon \sqrt{\mu_{A}^{i} X_{0}}, X_{i} \in\left[n_{1}\left(X_{0}\right), n_{2}\left(X_{0}\right)\right] \mid X_{0}>x\right) \\
& +\mathbb{P}\left(\left|S_{X_{i}}-S_{n_{0}\left(X_{0}\right)}\right|>\epsilon \sqrt{\mu_{A}^{i} X_{0}}, X_{i} \notin\left[n_{1}\left(X_{0}\right), n_{2}\left(X_{0}\right)\right] \mid X_{0}>x\right) \\
\leq & \mathbb{P}\left(\max _{n_{1}\left(X_{0}\right) \leq k \leq n_{2}\left(X_{0}\right)}\left|S_{k}-S_{n_{0}\left(X_{0}\right)}\right|>\epsilon \sqrt{\mu_{A}^{i} X_{0}} \mid X_{0}>x\right) \\
& +\mathbb{P}\left(X_{i} \notin\left[n_{1}\left(X_{0}\right), n_{2}\left(X_{0}\right)\right] \mid X_{0}>x\right) .
\end{aligned}
$$

Here, by Kolmogorov's theorem (see, e.g., Gut [17, Theorem 3.1.6]) and the independence of $X_{0}$ and $\tilde{A}_{j}^{(i+1)}, i \in\{0, \ldots, m\}, j \in \mathbb{N}$, we have for $x \geq 2 /\left(\mu_{A}^{i} \epsilon^{3}\right)$,

$$
\begin{aligned}
& \mathbb{P}\left(\max _{n_{1}\left(X_{0}\right) \leq k \leq n_{2}\left(X_{0}\right)}\left|S_{k}-S_{n_{0}\left(X_{0}\right)}\right|>\epsilon \sqrt{\mu_{A}^{i} X_{0}} \mid X_{0}>x\right) \\
& =\sum_{\ell>x, \ell \in \mathbb{N}} \frac{1}{\mathbb{P}\left(X_{0}>x\right)} \mathbb{P}\left(\max _{n_{1}(\ell) \leq k \leq n_{2}(\ell)}\left|S_{k}-S_{n_{0}(\ell)}\right|>\epsilon \sqrt{\mu_{A}^{i} \ell}, X_{0}=\ell\right) \\
& \leq \sum_{\ell>x, \ell \in \mathbb{N}} \frac{1}{\mathbb{P}\left(X_{0}>x\right)} \frac{\left(n_{2}(\ell)-n_{1}(\ell)\right) \sigma_{A}^{2}}{\epsilon^{2} \mu_{A}^{i} \ell} \mathbb{P}\left(X_{0}=\ell\right) \leq 2 \epsilon \sigma_{A}^{2} .
\end{aligned}
$$

Indeed, for $x \geq 2 /\left(\mu_{A}^{i} \epsilon^{3}\right)$, we have $n_{1}(\ell) \leq n_{0}(\ell) \leq n_{2}(\ell)$ for $\ell \geq x, \ell \in \mathbb{N}$, and

$$
\left|S_{k}-S_{n_{0}(\ell)}\right|= \begin{cases}\left|\sum_{j=n_{0}(\ell)+1}^{k} \tilde{A}_{j}^{(i+1)}\right| & \text { if } n_{0}(\ell) \leq k \leq n_{2}(\ell), \\ \left|\sum_{j=k+1}^{n_{0}(\ell)} \tilde{A}_{j}^{(i+1)}\right| & \text { if } n_{1}(\ell) \leq k \leq n_{0}(\ell)-1,\end{cases}
$$


so using the independence of $\tilde{A}_{j}^{(i+1)}, j \in \mathbb{N}$, by Kolmogorov's theorem, we have

$$
\begin{aligned}
& \mathbb{P}\left(\max _{n_{1}(\ell) \leq k \leq n_{2}(\ell)}\left|S_{k}-S_{n_{0}(\ell)}\right|>\epsilon \sqrt{\mu_{A}^{i} \ell}\right) \\
& \left.=\mathbb{P}\left(\max _{n_{0}(\ell) \leq k \leq n_{2}(\ell)}\left|\sum_{j=n_{0}(\ell)+1}^{k} \tilde{A}_{j}^{(i+1)}\right|{ }_{n_{1}(\ell) \leq k \leq n_{0}(\ell)-1}\left|\sum_{j=k+1}^{n_{0}(\ell)} \tilde{A}_{j}^{(i+1)}\right|\right)>\epsilon \sqrt{\mu_{A}^{i} \ell}\right) \\
& =1-\left(1-\mathbb{P}\left(\max _{n_{0}(\ell) \leq k \leq n_{2}(\ell) \mid}\left|\sum_{j=n_{0}(\ell)+1}^{k} \tilde{A}_{j}^{(i+1)}\right|>\epsilon \sqrt{\mu_{A}^{i} \ell}\right)\right) \\
& \quad \times\left(1-\mathbb{P}\left(\max _{n_{1}(\ell) \leq k \leq n_{0}(\ell)-1}\left|\sum_{j=k+1}^{n_{0}(\ell)} \tilde{A}_{j}^{(i+1)}\right|>\epsilon \sqrt{\mu_{A}^{i} \ell}\right)\right) \\
& \leq 1-\left(1-\frac{\left(n_{2}(\ell)-n_{0}(\ell)\right) \sigma_{A}^{2}}{\epsilon^{2} \mu_{A}^{i} \ell}\right)\left(1-\frac{\left(n_{0}(\ell)-n_{1}(\ell)\right) \sigma_{A}^{2}}{\epsilon^{2} \mu_{A}^{i} \ell}\right) \\
& =\frac{\left(n_{2}(\ell)-n_{1}(\ell)\right) \sigma_{A}^{2}}{\epsilon^{2} \mu_{A}^{i} \ell} \frac{\left(n_{2}(\ell)-n_{0}(\ell)\right)\left(n_{0}(\ell)-n_{1}(\ell)\right) \sigma_{A}^{2}}{\epsilon^{4} \mu_{A}^{2 i} \ell^{2}} \\
& \leq \frac{\left(n_{2}(\ell)-n_{1}(\ell)\right) \sigma_{A}^{2}}{\epsilon^{2} \mu_{A}^{i} \ell},
\end{aligned}
$$

yielding the first inequality in (2.10). The second inequality in (2.10) follows by

$$
\frac{n_{2}(\ell)-n_{1}(\ell)}{\ell} \leq \frac{\left(1+\epsilon^{3}\right) \mu_{A}^{i} \ell-\left(1-\epsilon^{3}\right) \mu_{A}^{i} \ell}{\ell}=2 \mu_{A}^{i} \epsilon^{3} .
$$

Consequently, for all $\epsilon \in(0,1)$,

$$
\mathbb{P}\left(\left|\Delta_{i}\right|>\epsilon \mid X_{0}>x\right) \leq 2 \epsilon \sigma_{A}^{2}+\mathbb{P}\left(X_{i} \notin\left[n_{1}\left(X_{0}\right), n_{2}\left(X_{0}\right)\right] \mid X_{0}>x\right)
$$

Here

$$
\begin{aligned}
& \mathbb{P}\left(X_{i} \notin\left[n_{1}\left(X_{0}\right), n_{2}\left(X_{0}\right)\right] \mid X_{0}>x\right) \\
& \leq \mathbb{P}\left(X_{i} \notin\left[\left(1-\epsilon^{3}\right) \mu_{A}^{i} X_{0}+1,\left(1+\epsilon^{3}\right) \mu_{A}^{i} X_{0}-1\right] \mid X_{0}>x\right) \\
& =\mathbb{P}\left(\left(\mu_{A}^{i} X_{0}\right)^{-1} X_{i}-1 \notin\left[-\epsilon^{3}+\left(\mu_{A}^{i} X_{0}\right)^{-1}, \epsilon^{3}-\left(\mu_{A}^{i} X_{0}\right)^{-1}\right] \mid X_{0}>x\right) \\
& \leq \mathbb{P}\left(\left(\mu_{A}^{i} X_{0}\right)^{-1} X_{i}-1 \notin\left[-\epsilon^{3}+\left(\mu_{A}^{i} x\right)^{-1}, \epsilon^{3}-\left(\mu_{A}^{i} x\right)^{-1}\right] \mid X_{0}>x\right) \\
& \leq \mathbb{P}\left(\left(\mu_{A}^{i} X_{0}\right)^{-1} X_{i}-1 \notin\left[-\epsilon^{3} / 2, \epsilon^{3} / 2\right] \mid X_{0}>x\right)
\end{aligned}
$$

if $\left(\mu_{A}^{i} x\right)^{-1} \leq \epsilon^{3} / 2$, i.e., for $x \geq 2 /\left(\mu_{A}^{i} \epsilon^{3}\right)$, hence

$$
\mathbb{P}\left(X_{i} \notin\left[n_{1}\left(X_{0}\right), n_{2}\left(X_{0}\right)\right] \mid X_{0}>x\right) \rightarrow 0 \quad \text { as } x \rightarrow \infty,
$$


due to (2.4). Consequently, we have (2.9). By (2.8) and part (i) of Lemma D.4, we obtain

$$
\mathcal{L}\left(0, \ldots, 0, \Delta_{0}, \ldots, \Delta_{m} \mid X_{0}>x\right) \stackrel{w}{\longrightarrow} \delta_{(0, \ldots, 0,0, \ldots, 0)} \quad \text { as } x \rightarrow \infty .
$$

Hence, using (2.7) and Lemma D.5, we obtain (2.2) with $W_{i}^{\prime}$ replaced by $W_{i}, i \in\{0, \ldots, m\}$, as desired.

\section{Point process convergence}

Let $\left(a_{n}\right)_{n \in \mathbb{N}}$ be a sequence of positive real numbers satisfying $\lim _{n \rightarrow \infty} a_{n}=\infty$ and

$$
\lim _{n \rightarrow \infty} n \mathbb{P}\left(X_{0}>a_{n}\right)=1
$$

Note that for $n \geq 2$, one can choose $a_{n}$ to be the maximum of 1 and the $1-\frac{1}{n}$ lower quantile of $X_{0}$. In fact, $a_{n}=n^{1 / \alpha} L(n), n \in \mathbb{N}$, for some slowly varying continuous function $L:(0, \infty) \rightarrow(0, \infty)$, see, e.g., Araujo and Giné [1, Exercise 6 on page 90]. In what follows we fix such a sequence $\left(a_{n}\right)_{n \in \mathbb{N}}$ satisfying (3.1). By the proof of Lemma 3.2 in Basrak et al. [5], for any sequence of positive integers $\left(r_{n}\right)_{n \geq 1}$ such that $\lim _{n \rightarrow \infty} r_{n}=\infty$ and $\lim _{n \rightarrow \infty} n / r_{n}=\infty$, we have

$$
\lim _{m \rightarrow \infty} \limsup _{n \rightarrow \infty} \mathbb{P}\left(\max _{m<|i| \leq r_{n}} X_{i}>a_{n} u \mid X_{0}>a_{n} u\right)=0 \quad \text { for all } u>0 .
$$

Condition (3.2) is sometimes called the anticlustering condition or finite mean cluster size condition for the strongly stationary process $\left(X_{i}\right)_{i \in \mathbb{Z}}$. Later, in the proof of Theorem 3.2 we will choose a particular sequence $\left(r_{n}\right)_{n \in \mathbb{N}}$ satisfying a kind of mixing condition (3.6) as well. For a possible choice of such a sequence $\left(r_{n}\right)_{n \in \mathbb{N}}$, see the proof of Lemma C.2.

Consider the space $S:=(0, \infty) \times \mathbb{R}$ with the usual topology and call a Borel set $B \subset S$ bounded if it is separated from the vertical line $\{(0, y): y \in \mathbb{R}\}$, i.e., there exists $\epsilon>0$ such that $B \subset\{(x, y) \in(0, \infty) \times \mathbb{R}: x>\epsilon\}$. Using the terminology of Basrak and Planinić [6], the collection of bounded sets is a boundedness (Borel subsets of bounded sets are bounded, and finite union of bounded sets are bounded), which properly localizes $S$. Denote by $\mathcal{B}(S)$ the Borel $\sigma$-algebra on $S$ and by $\widehat{C}_{S}$ the class of bounded, continuous functions $f: S \rightarrow[0, \infty)$ with bounded support. Hence, if $f \in \widehat{C}_{S}$, then there exists an $\epsilon>0$ such that $f(x, y)=0$ for all $(x, y) \in S$ with $x \leq \epsilon$.

Let $\mathcal{M}_{p}(S)$ be the space of integer-valued measures (or point measures) on $S$ which are finite on bounded Borel sets (called locally finite measures), topologized by the so-called vague topology. The associated notion of vague convergence of $\mu_{n} \in \mathcal{M}_{p}(S)$ towards $\mu \in \mathcal{M}_{p}(S)$ as $n \rightarrow \infty$, denoted by $\mu_{n} \stackrel{v}{\longrightarrow} \mu$ as $n \rightarrow \infty$, is defined by the condition $\mu_{n}(f) \rightarrow \mu(f)$ as $n \rightarrow \infty$

for all $f \in \widehat{C}_{S}$, where $\kappa(f):=\int_{S} f(x, y) \kappa(\mathrm{d} x, \mathrm{~d} y)$ for each $\kappa \in \mathcal{M}_{p}(S)$. Alternatively, we could have used the framework of so-called $\mathbb{M}_{\mathbb{O}}-$ convergence from Lindskog et al. [31], see also Basrak and Planinić [6] for the discussion on vague convergence. Convergence in distribution of random measures with respect to the vague topology will be denoted by $\stackrel{v d}{\longrightarrow}$. 
In what follows, we use the theory of Kallenberg [21, Chapter 4]. To do so, one can equip $S$ with the metric $d: S \times S \rightarrow[0, \infty)$,

$$
d\left((x, y),\left(x^{\prime}, y^{\prime}\right)\right):=\min \left(\sqrt{\left(x-x^{\prime}\right)^{2}+\left(y-y^{\prime}\right)^{2}}, 1\right)+\left|\frac{1}{x}-\frac{1}{x^{\prime}}\right|
$$

for $(x, y),\left(x^{\prime}, y^{\prime}\right) \in S$. This metric is complete and induces the original topology of $S$, and the family of $d$-bounded sets is precisely the family of bounded sets in $S$ as defined above, see Lemma A.1. In Kallenberg [21, page 125], one can find a similar metric. Note that $\mathcal{M}_{p}(S)$ is a complete separable metric space with a metric inducing the vague topology, see Kallenberg [21, Lemma 4.6].

The following well-known result (stated in our setting) describes vague convergence of point measures and is crucial for the use of continuous mapping arguments (for a proof, based on Lemma 3.13 in Resnick [35], see Appendix B), we will use it in the proof of Theorem 4.1.

Lemma 3.1. Let $\mu, \mu_{n} \in \mathcal{M}_{p}(S), n \in \mathbb{N}$. Then $\mu_{n} \stackrel{v}{\longrightarrow} \mu$ as $n \rightarrow \infty$ if and only if for each $\epsilon>0$ satisfying $\mu(\{\epsilon\} \times \mathbb{R})=0$ there exist integers $n_{0}, M \geq 0$ and a labeling of the points of $\mu$ and $\mu_{n}, n \geq n_{0}$, in $(\epsilon, \infty) \times \mathbb{R}$ such that

$$
\left.\mu_{n}\right|_{(\epsilon, \infty) \times \mathbb{R}}=\sum_{i=1}^{M} \delta_{\left(x_{i}^{(n)}, y_{i}^{(n)}\right)},\left.\quad \mu\right|_{(\epsilon, \infty) \times \mathbb{R}}=\sum_{i=1}^{M} \delta_{\left(x_{i}, y_{i}\right)},
$$

and $x_{i}^{(n)} \rightarrow x_{i}$ and $y_{i}^{(n)} \rightarrow y_{i}$ as $n \rightarrow \infty$ for all $i=1, \ldots, M$, where $\left.\mu\right|_{B}$ denotes the restriction of $\mu$ to the set $B \subset S$.

Recall (2.1) and define

$$
\theta:=\mathbb{P}\left(\sup _{j<0} Y_{j} \leq 1\right)=\mathbb{P}(K=0)=1-\mu_{A}^{\alpha} .
$$

Indeed, $\left\{\sup _{j<0} Y_{j} \leq 1\right\}=\{K=0\}$, since if $K=0$, then, by (2.1), $Y_{j}=0$ for all $j<0$ yielding $\sup _{j<0} Y_{j}=0 \leq 1$; and if $K \geq 1$, then $\sup _{j<0} Y_{j} \geq Y_{-1}=\mu_{A}^{-1} Y_{0}>1$ almost surely due to the facts that $\mathbb{P}\left(Y_{0} \geq 1\right)=1$ and $\mu_{A} \in(0,1)$.

Theorem 3.2. In $\mathcal{M}_{p}(S)$,

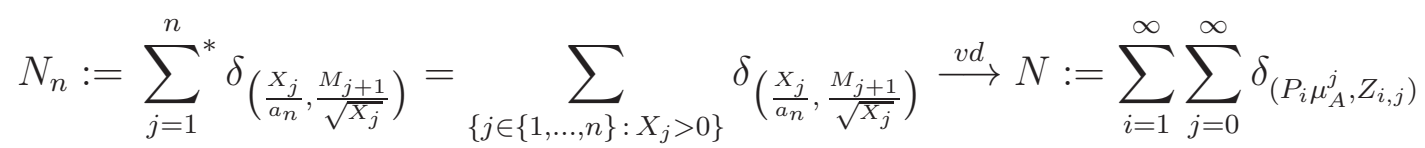

as $n \rightarrow \infty$, where $\sum_{i=1}^{\infty} \delta_{P_{i}}$ denotes a Poisson point process on $(0, \infty)$ with intensity $\theta \mathrm{d}\left(-y^{-\alpha}\right)$ which is independent of an i.i.d. array of $\mathcal{N}\left(0, \sigma_{A}^{2}\right)$-distributed random variables $\left\{Z_{i, j}: i \in\right.$ $\mathbb{N}, j \geq 0\}$.

Proof. First, we check that $N$ is a locally finite measure almost surely, i.e., $N(B)$ is finite almost surely for each bounded Borel measurable subset $B$ of $S$. Assume that $B \in \mathcal{B}(S)$ is bounded. Then we have $B \subset(\epsilon, \infty) \times \mathbb{R}$ for some $\epsilon>0$, and

$$
N((\epsilon, \infty) \times \mathbb{R})=\sum_{i=1}^{\infty} \sum_{j=0}^{\infty} \mathbb{1}_{\left\{\left(P_{i} \mu_{A}^{j}, Z_{i, j}\right) \in(\epsilon, \infty) \times \mathbb{R}\right\}}=\sum_{j=0}^{\infty} \sum_{i=1}^{\infty} \mathbb{1}_{\left\{P_{i}>\mu_{A}^{-j} \epsilon\right\}}<\infty
$$


almost surely, since, for each $j \geq 0$, there are only finitely many $P_{i}^{\prime}$ 's greater than $\mu_{A}^{-j} \epsilon$ almost surely, and for sufficiently large $j \geq 0$, the set $\left\{i \in \mathbb{N}: P_{i}>\mu_{A}^{-j} \epsilon\right\}$ is empty, since $\mu_{A}^{-j} \epsilon \geq$ $\max _{i \in \mathbb{N}} P_{i}$ holds for sufficiently large $j \geq 0$ due to $\mu_{A}^{-j} \rightarrow \infty$ as $j \rightarrow \infty$.

By Remark 3.1 in Basrak et al. [5] or Lemma F.1 in Barczy et al. [3], the strongly stationary process $\left(X_{j}\right)_{j \in \mathbb{Z}}$ is strongly mixing, and since $M_{j+1} / \sqrt{X_{j}} \in \sigma\left(X_{j}, X_{j+1}\right)$ for every $j \geq 0$ (understanding this ratio 0 when $X_{j}=0$ ), a modification of Lemma 2.3.9 in Basrak [4] or the proof of Proposition 1.34 in Krizmanić [25], shows that there exists a sequence of positive integers $\left(r_{n}\right)_{n \in \mathbb{N}}$ satisfying $r_{n} \rightarrow \infty$ and $r_{n} / n \rightarrow 0$ as $n \rightarrow \infty$, such that

$$
\mathbb{E}\left[\exp \left\{-\sum_{j=1}^{n} f\left(\frac{X_{j}}{a_{n}}, \frac{M_{j+1}}{\sqrt{X_{j}}}\right)\right\}\right]-\left(\mathbb{E}\left[\exp \left\{-\sum_{j=1}^{r_{n}} *\left(\frac{X_{j}}{a_{n}}, \frac{M_{j+1}}{\sqrt{X_{j}}}\right)\right\}\right]\right)^{k_{n}} \rightarrow 0
$$

as $n \rightarrow \infty$ for all $f \in \widehat{C}_{S}$, where $k_{n}:=\left\lfloor n / r_{n}\right\rfloor$, see Lemma C.2. This is similar to condition (2.1) in Davis and Hsing [11]. Note that for this sequence $\left(r_{n}\right)_{n \in \mathbb{N}}$, the anticlustering condition (3.2) holds automatically, as explained before (3.2).

By Theorem 4.11 in Kallenberg [21] and (3.6), $N_{n} \stackrel{v d}{\longrightarrow} N$ as $n \rightarrow \infty$ if and only if $\widetilde{N}_{n}:=$ $\sum_{i=1}^{k_{n}} \widetilde{N}_{n, i} \stackrel{v d}{\longrightarrow} N$ as $n \rightarrow \infty$, where for each $n \in \mathbb{N}, \widetilde{N}_{n, i}, i=1, \ldots, k_{n}$, are i.i.d. point processes on $S$ with common distribution equal to the distribution of

$$
N_{r_{n}}=\sum_{j=1}^{r_{n}} \delta^{*}\left(\frac{x_{j}}{a_{n}}, \frac{M_{j+1}}{\sqrt{X_{j}}}\right)
$$

since (3.6) takes the form

$$
\mathbb{E}\left[\mathrm{e}^{-N_{n}(f)}\right]-\mathbb{E}\left[\mathrm{e}^{-\widetilde{N}_{n}(f)}\right] \rightarrow 0
$$

as $n \rightarrow \infty$ for all $f \in \widehat{C}_{S}$.

We will apply Theorem 4.22 in Kallenberg [21]. The array of point processes $\left\{\widetilde{N}_{n, i}, n \in\right.$ $\left.\mathbb{N}, i=1, \ldots, k_{n}\right\}$ forms a null array, since if $B \subset S$ is a bounded Borel set, then there exists an $\epsilon>0$ such that $B \subset(\epsilon, \infty) \times \mathbb{R}$, and then

$$
\begin{aligned}
& \sup _{i \in\left\{1, \ldots, k_{n}\right\}} \mathbb{P}\left(\widetilde{N}_{n, i}(B)>0\right)=\mathbb{P}\left(\widetilde{N}_{n, 1}(B)>0\right)=\mathbb{P}\left(\sum_{j=1}^{r_{n}} \delta_{\left(X_{j} / a_{n}, M_{j+1} / \sqrt{X_{j}}\right)}(B)>0\right) \\
& \leq \mathbb{P}\left(\bigcup_{j=1}^{r_{n}}\left\{\left(\frac{X_{j}}{a_{n}}, \frac{M_{j+1}}{\sqrt{X_{j}}}\right) \in(\epsilon, \infty) \times \mathbb{R}\right\}\right) \leq \sum_{j=1}^{r_{n}} \mathbb{P}\left(\frac{X_{j}}{a_{n}} \in(\epsilon, \infty)\right) \\
& =r_{n} \mathbb{P}\left(\frac{X_{1}}{a_{n}} \in(\epsilon, \infty)\right)=\frac{r_{n}}{n} \cdot n \mathbb{P}\left(X_{1}>a_{n}\right) \cdot \frac{\mathbb{P}\left(X_{1}>\epsilon a_{n}\right)}{\mathbb{P}\left(X_{1}>a_{n}\right)} \rightarrow 0 \cdot 1 \cdot \epsilon^{-\alpha}=0 \quad \text { as } n \rightarrow \infty,
\end{aligned}
$$

where the last step follows by (3.1) and the fact that $X_{1}$ is regularly varying with tail index $\alpha$. By Kallenberg [21, Theorem 4.22 and page 89], $\widetilde{N}_{n} \stackrel{v d}{\longrightarrow} N$ as $n \rightarrow \infty$ if there exists a measure $\nu$ on $\mathcal{M}_{p}(S) \backslash\{0\}$ (furnished with the smallest $\sigma$-algebra making all the evaluation maps $\mathcal{M}_{p}(S) \ni m \mapsto m(F)$ measurable, where $\left.F \in \mathcal{B}(S)\right)$ such that 
(i) $\int_{\mathcal{M}_{p}(S) \backslash\{0\}} \min (\kappa(B), 1) \nu(\mathrm{d} \kappa)<\infty$ for all bounded Borel subsets of $S$,

(ii) $k_{n} \mathbb{E}\left[1-\mathrm{e}^{-N_{r_{n}}(f)}\right] \rightarrow \int_{\mathcal{M}_{p}(S) \backslash\{0\}}\left(1-\mathrm{e}^{-\kappa(f)}\right) \nu(\mathrm{d} \kappa)$ as $n \rightarrow \infty$ for all $f \in \widehat{C}_{S}$,

(iii) $-\log \mathbb{E}\left[\mathrm{e}^{-N(f)}\right]=\int_{\mathcal{M}_{p}(S) \backslash\{0\}}\left(1-\mathrm{e}^{-\kappa(f)}\right) \nu(\mathrm{d} \kappa)$ for all $f \in \widehat{C}_{S}$.

Here we note that, with the notations of Kallenberg [21], the non-random locally finite measure $\alpha$ on $S$ appearing in Theorem 4.22 in Kallenberg [21] is the null measure, by Theorem 3.20 in Kallenberg [21].

Let $\nu$ be a measure on $\mathcal{M}_{p}(S) \backslash\{0\}$ given by

$$
\nu(\cdot):=\theta \int_{0}^{\infty} \mathbb{P}\left(\sum_{j=0}^{\infty} \delta_{\left(y \mu_{A}^{j}, Z_{j}\right)} \in \cdot\right) \alpha y^{-\alpha-1} \mathrm{~d} y,
$$

where $\left(Z_{j}\right)_{j \geq 0}$ is an i.i.d. sequence of $\mathcal{N}\left(0, \sigma_{A}^{2}\right)$-distributed random variables being independent of $Y_{0}$ and $K$ with $\sigma_{A}^{2}=\operatorname{Var}(A)$. Note that we have

$$
\int_{\mathcal{M}_{p}(S) \backslash\{0\}} h(\kappa(g)) \nu(\mathrm{d} \kappa)=\theta \int_{0}^{\infty} \mathbb{E}\left[h\left(\sum_{j=0}^{\infty} g\left(y \mu_{A}^{j}, Z_{j}\right)\right)\right] \alpha y^{-\alpha-1} \mathrm{~d} y
$$

for any measurable functions $h: \mathbb{R} \rightarrow[0, \infty)$ and $g: S \rightarrow[0, \infty)$ such that $g$ has bounded support, since this trivially holds for linear combinations of indicator functions, which extends by monotone convergence to arbitrary nonnegative measurable functions. Consequently, $\nu$ satisfies property (i) above, since if $B$ is a bounded Borel subset of $S$, then there exists $\epsilon>0$ such that $x>\epsilon$ for all $(x, z) \in B$, and hence

$$
\begin{aligned}
& \int_{\mathcal{M}_{p}(S) \backslash\{0\}} \min (\kappa(B), 1) \nu(\mathrm{d} \kappa)=\theta \int_{0}^{\infty} \mathbb{E}\left[\left(\sum_{j=0}^{\infty} \mathbb{1}_{B}\left(y \mu_{A}^{j}, Z_{j}\right)\right) \wedge 1\right] \alpha y^{-\alpha-1} \mathrm{~d} y \\
& =\theta \int_{y>\epsilon}^{\infty} \mathbb{E}\left[\left(\sum_{j=0}^{\infty} \mathbb{1}_{B}\left(y \mu_{A}^{j}, Z_{j}\right)\right) \wedge 1\right] \alpha y^{-\alpha-1} \mathrm{~d} y \leq \theta \int_{y>\epsilon}^{\infty} \alpha y^{-\alpha-1} \mathrm{~d} y=\theta \epsilon^{-\alpha}<\infty,
\end{aligned}
$$

where we used that if $y \in(0, \epsilon]$, then $y \mu_{A}^{j} \in(0, \epsilon], j \geq 0$.

Next we turn to prove (iii). We check that $N$ given in (3.5) satisfies

$$
-\log \mathbb{E}\left[\mathrm{e}^{-N(f)}\right]=\theta \int_{0}^{\infty} \mathbb{E}\left[1-\mathrm{e}^{-\sum_{j=0}^{\infty} f\left(y \mu_{A}^{j}, Z_{j}\right)}\right] \alpha y^{-\alpha-1} \mathrm{~d} y=\int_{\mathcal{M}_{p}(S) \backslash\{0\}}\left(1-\mathrm{e}^{-\kappa(f)}\right) \nu(\mathrm{d} \kappa)
$$

for all $f \in \widehat{C}_{S}$. The second equality follows from (3.7). For the first equality, we provide two alternative proofs. The first equality in (3.8) can be derived using the representation

$$
N(f)=\sum_{i=1}^{\infty} \sum_{j=0}^{\infty} f\left(P_{i} \mu_{A}^{j}, Z_{i, j}\right)=\sum_{i=1}^{\infty} f^{\prime}\left(P_{i},\left(Z_{i, j}\right)_{j \geq 0}\right)=N^{\prime}\left(f^{\prime}\right)
$$


where $f^{\prime}\left(p,\left(z_{j}\right)_{j \geq 0}\right):=\sum_{j=0}^{\infty} f\left(p \mu_{A}^{j}, z_{j}\right)$ for $p \in(0, \infty), z_{j} \in \mathbb{R}, j \geq 0$, and $N^{\prime}:=\sum_{i=1}^{\infty} \delta_{\left(P_{i},\left(Z_{i, j}\right)_{j \geq 0}\right)}$ is a Poisson point process on $(0, \infty) \times \mathbb{R}^{\mathbb{N} \cup\{0\}}$ with intensity $\theta \mathrm{d}\left(-y^{-\alpha}\right) \times \mathcal{L}\left(Z_{1,1}\right)^{\mathbb{N} \cup\{0\}}$ (see, e.g., Kingman [23, Section 5.2]). Indeed, $f \in \widehat{C}_{S}$ implies that the sum $\sum_{j=0}^{\infty} f\left(p \mu_{A}^{j}, z_{j}\right)$ has only finitely many non-zero terms, and one can use the expression for the Laplace functional of $N^{\prime}$ together with Fubini's theorem. The second proof of the first equality in (3.8) is based on the representation

$$
\begin{aligned}
\mathbb{E}\left[\mathrm{e}^{-N(f)}\right] & =\mathbb{E}\left[\mathbb{E}\left[\mathrm{e}^{-\sum_{i=1}^{\infty} \sum_{j=0}^{\infty} f\left(P_{i} \mu_{A}^{j}, Z_{i, j}\right)} \mid\left(P_{i}\right)_{i \in \mathbb{N}}\right]\right] \\
& =\mathbb{E}\left[\prod_{i=1}^{\infty} \prod_{j=0}^{\infty} \mathbb{E}\left[\mathrm{e}^{-f\left(P_{i} \mu_{A}^{j}, Z\right)} \mid\left(P_{i}\right)_{i \in \mathbb{N}}\right]\right]=\mathbb{E}\left[\prod_{i=1}^{\infty} h\left(P_{i}\right)\right],
\end{aligned}
$$

where $Z$ is an $\mathcal{N}\left(0, \sigma_{A}^{2}\right)$-distributed random variable, independent of $\left(P_{i}\right)_{i \in \mathbb{N}}$, and $h:(0, \infty) \rightarrow \mathbb{R}$ is defined by

$$
h(x):=\prod_{j=0}^{\infty} \mathbb{E}\left[\mathrm{e}^{-f\left(x \mu_{A}^{j}, Z\right)}\right], \quad x \in(0, \infty) .
$$

Consequently, using the Laplace functional of the Poisson point process $\sum_{i=1}^{\infty} \delta_{P_{i}}$, we obtain

$$
\begin{aligned}
\mathbb{E}\left[\mathrm{e}^{-N(f)}\right] & =\mathbb{E}\left[\mathrm{e}^{-\sum_{i=1}^{\infty}\left(-\log \left(h\left(P_{i}\right)\right)\right)}\right]=\exp \left\{-\int_{0}^{\infty}\left(1-\mathrm{e}^{-(-\log (h(x)))}\right) \theta \alpha x^{-\alpha-1} \mathrm{~d} x\right\} \\
& =\exp \left\{-\theta \int_{0}^{\infty}(1-h(x)) \alpha x^{-\alpha-1} \mathrm{~d} x\right\} \\
& =\exp \left\{-\theta \int_{0}^{\infty}\left(1-\prod_{j=0}^{\infty} \mathbb{E}\left[\mathrm{e}^{-f\left(x \mu_{A}^{j}, Z\right)}\right]\right) \alpha x^{-\alpha-1} \mathrm{~d} x\right\} \\
& =\exp \left\{-\theta \int_{0}^{\infty}\left(1-\mathbb{E}\left[\mathrm{e}^{-\sum_{j=0}^{\infty} f\left(x \mu_{A}^{j}, Z_{j}\right)}\right]\right) \alpha x^{-\alpha-1} \mathrm{~d} x\right\},
\end{aligned}
$$

as desired.

Now we prove (ii). By (3.8), we need to show that

$$
k_{n} \mathbb{E}\left[1-\mathrm{e}^{-N_{r_{n}}(f)}\right] \rightarrow \theta \int_{0}^{\infty} \mathbb{E}\left[1-\mathrm{e}^{-\sum_{j=0}^{\infty} f\left(y \mu_{A}^{j}, Z_{j}\right)}\right] \alpha y^{-\alpha-1} \mathrm{~d} y
$$

as $n \rightarrow \infty$ for all $f \in \widehat{C}_{S}$. Take an arbitrary $f \in \widehat{C}_{S}$ and let $\epsilon>0$ be such that $x \leq \epsilon$ implies that $f(x, y)=0$ for all $y \in \mathbb{R}$. To show (3.9) we rely on the condition (3.2) and use similar arguments as in the proof of Basrak and Segers [7, Theorem 4.3]. Note that, even though condition (3.2) concerns only the process $\left(X_{j}\right)_{j \in \mathbb{Z}}$, it will be sufficient for (3.9), since the bounded sets in $S$ are separated from the vertical line (see Lemma A.1) by our choice of bounded sets in $S$.

For $n \in \mathbb{N}$ and $k, \ell \in \mathbb{Z}$ with $k \leq \ell$, write $M_{k, \ell}:=\max _{k \leq j \leq \ell} X_{j}$ (if $\ell<k$ set $M_{k, \ell}:=0$ ), and

$$
c_{n}(k, \ell):=1-\exp \left\{-\sum_{j=k}^{\ell} f\left(\frac{X_{j}}{a_{n}}, \frac{M_{j+1}}{\sqrt{X_{j}}}\right)\right\} \text {. }
$$


In particular, $c_{n}\left(1, r_{n}\right)=1-\exp \left\{-N_{r_{n}}(f)\right\}$, and since $f(x, y)=0$ if $(x, y) \in(0, \epsilon] \times \mathbb{R}$, we have $\left\{M_{1, r_{n}} \leq a_{n} \epsilon\right\} \subset\left\{c_{n}\left(1, r_{n}\right)=0\right\}$, and then

$$
\mathbb{E}\left[c_{n}\left(1, r_{n}\right)\right]=\mathbb{E}\left[c_{n}\left(1, r_{n}\right) \mathbb{1}_{\left\{M_{1, r_{n}}>a_{n} \epsilon\right\}}\right]=\sum_{i=1}^{r_{n}} \mathbb{E}\left[c_{n}\left(1, r_{n}\right) \mathbb{1}_{\left\{M_{1, i-1} \leq a_{n} \epsilon<X_{i}\right\}}\right] .
$$

Since $k_{n} \sim\left(r_{n} \mathbb{P}\left(X_{0}>a_{n}\right)\right)^{-1}$ as $n \rightarrow \infty$, (3.9) will follow if we show that

$$
\frac{\mathbb{E}\left[c_{n}\left(1, r_{n}\right)\right]}{r_{n} \mathbb{P}\left(X_{0}>a_{n}\right)} \rightarrow \theta \int_{0}^{\infty} \mathbb{E}\left[1-\mathrm{e}^{-\sum_{j=0}^{\infty} f\left(y \mu_{A}^{j}, Z_{j}\right)}\right] \alpha y^{-\alpha-1} \mathrm{~d} y \quad \text { as } n \rightarrow \infty .
$$

Fix now $m \in \mathbb{N}$ and assume that $n$ is large enough so that $r_{n} \geq 2 m+1$. The key observation now is that, since $f(x, y)=0$ if $x \leq \epsilon$, for all $m+1 \leq i \leq r_{n}-m$, we have $c_{n}\left(1, r_{n}\right)=c_{n}(i-m, i+m)$ if $M_{1, i-m-1} \vee M_{i+m+1, r_{n}} \leq a_{n} \epsilon$. Using the strong stationarity of $\left(X_{i}\right)_{i \in \mathbb{Z}}$ and that $c_{n}(k, l) \in[0,1]$, $n \in \mathbb{N}, k, l \in \mathbb{Z}$, this implies that for all $i \in\left\{m+1, \ldots, r_{n}-m\right\}$,

$$
\begin{aligned}
& \left|\mathbb{E}\left[c_{n}\left(1, r_{n}\right) \mathbb{1}_{\left\{M_{1, i-1} \leq a_{n} \epsilon<X_{i}\right\}}\right]-\mathbb{E}\left[c_{n}(i-m, i+m) \mathbb{1}_{\left\{M_{i-m, i-1} \leq a_{n} \epsilon<X_{i}\right\}}\right]\right| \\
& \leq\left|\mathbb{E}\left[\left(c_{n}\left(1, r_{n}\right) \mathbb{1}_{\left\{M_{1, i-1} \leq a_{n} \epsilon<X_{i}\right\}}-c_{n}(i-m, i+m) \mathbb{1}_{\left\{M_{i-m, i-1} \leq a_{n} \epsilon<X_{i}\right\}}\right) \mathbb{1}_{\left\{M_{1, i-m-1} \vee M_{i+m+1, r_{n}} \leq a_{n} \epsilon\right\}}\right]\right| \\
& +\left|\mathbb{E}\left[\left(c_{n}\left(1, r_{n}\right) \mathbb{1}_{\left\{M_{1, i-1} \leq a_{n} \epsilon<X_{i}\right\}}-c_{n}(i-m, i+m) \mathbb{1}_{\left\{M_{i-m, i-1} \leq a_{n} \epsilon<X_{i}\right\}}\right) \mathbb{1}_{\left\{M_{1, i-m-1} \vee M_{i+m+1, r_{n}}>a_{n} \epsilon\right\}}\right]\right| \\
& =\left|\mathbb{E}\left[\left(c_{n}\left(1, r_{n}\right)-c_{n}(i-m, i+m)\right) \mathbb{1}_{\left\{M_{1, i-1} \leq a_{n} \epsilon<X_{i}\right\}} \mathbb{1}_{\left\{M_{1, i-m-1} \vee M_{i+m+1, r_{n}} \leq a_{n} \epsilon\right\}}\right]\right| \\
& +\left|\mathbb{E}\left[\left(c_{n}\left(1, r_{n}\right) \mathbb{1}_{\left\{M_{1, i-1} \leq a_{n} \epsilon\right\}}-c_{n}(i-m, i+m) \mathbb{1}_{\left\{M_{i-m, i-1} \leq a_{n} \epsilon\right\}}\right) \mathbb{1}_{\left\{X_{i}>a_{n} \epsilon\right\}} \mathbb{1}_{\left\{M_{1, i-m-1} \vee M_{i+m+1, r_{n}}>a_{n} \epsilon\right\}}\right]\right| \\
& \leq \mathbb{P}\left(M_{1, i-m-1} \vee M_{i+m+1, r_{n}}>a_{n} \epsilon, X_{i}>a_{n} \epsilon\right) \\
& =\mathbb{P}\left(M_{1-i,-m-1} \vee M_{m+1, r_{n}-i}>a_{n} \epsilon, X_{0}>a_{n} \epsilon\right) \\
& \leq \mathbb{P}\left(M_{-r_{n},-m-1} \vee M_{m+1, r_{n}}>a_{n} \epsilon, X_{0}>a_{n} \epsilon\right),
\end{aligned}
$$

where the last step follows by $-r_{n} \leq 1-i$ and $r_{n}-i \leq r_{n}$. For $i \in\{1, \ldots, m\} \cup\left\{r_{n}-m+\right.$ $\left.1, \ldots, r_{n}\right\}$, use the trivial bound

$$
\begin{aligned}
& \left|\mathbb{E}\left[c_{n}\left(1, r_{n}\right) \mathbb{1}_{\left\{M_{1, i-1} \leq a_{n} \epsilon<X_{i}\right\}}\right]-\mathbb{E}\left[c_{n}(i-m, i+m) \mathbb{1}_{\left\{M_{i-m, i-1} \leq a_{n} \epsilon<X_{i}\right\}}\right]\right| \\
& \leq \mathbb{E}\left[\left|c_{n}\left(1, r_{n}\right) \mathbb{1}_{\left\{M_{1, i-1} \leq a_{n} \epsilon\right\}}-c_{n}(i-m, i+m) \mathbb{1}_{\left\{M_{i-m, i-1} \leq a_{n} \epsilon\right\}}\right| \mathbb{1}_{\left\{a_{n} \epsilon<X_{i}\right\}}\right] \leq \mathbb{P}\left(X_{0}>a_{n} \epsilon\right) .
\end{aligned}
$$

Consequently, using again the strong stationarity of $\left(X_{i}\right)_{i \in \mathbb{Z}}$ and (3.10), we have

$$
\begin{aligned}
& \Delta_{n, m}:=\left|\frac{\mathbb{E}\left[c_{n}\left(1, r_{n}\right)\right]}{r_{n} \mathbb{P}\left(X_{0}>a_{n}\right)}-\frac{\mathbb{E}\left[c_{n}(-m, m) \mathbb{1}_{\left\{M_{-m,-1} \leq a_{n} \epsilon<X_{0}\right\}}\right]}{\mathbb{P}\left(X_{0}>a_{n}\right)}\right| \\
& =\frac{1}{r_{n} \mathbb{P}\left(X_{0}>a_{n}\right)}\left|\sum_{i=1}^{r_{n}} \mathbb{E}\left[c_{n}\left(1, r_{n}\right) \mathbb{1}_{\left\{M_{1, i-1} \leq a_{n} \epsilon<X_{i}\right\}}\right]-r_{n} \mathbb{E}\left[c_{n}(-m, m) \mathbb{1}_{\left\{M_{-m,-1} \leq a_{n} \epsilon<X_{0}\right\}}\right]\right| \\
& =\frac{1}{r_{n} \mathbb{P}\left(X_{0}>a_{n}\right)}\left|\sum_{i=1}^{r_{n}} \mathbb{E}\left[c_{n}\left(1, r_{n}\right) \mathbb{1}_{\left\{M_{1, i-1} \leq a_{n} \epsilon<X_{i}\right\}}\right]-\sum_{i=1}^{r_{n}} \mathbb{E}\left[c_{n}(i-m, i+m) \mathbb{1}_{\left\{M_{i-m, i-1} \leq a_{n} \epsilon<X_{i}\right\}}\right]\right|
\end{aligned}
$$




$$
\begin{aligned}
\leq & \frac{1}{r_{n} \mathbb{P}\left(X_{0}>a_{n}\right)}\left(\sum_{i \in\{1, \ldots, m\} \cup\left\{r_{n}-m+1, \ldots, r_{n}\right\}} \mathbb{P}\left(X_{0}>a_{n} \epsilon\right)\right. \\
& \left.\quad+\sum_{i=m+1}^{r_{n}-m} \mathbb{P}\left(M_{-r_{n},-m-1} \vee M_{m+1, r_{n}}>a_{n} \epsilon, X_{0}>a_{n} \epsilon\right)\right) \\
= & \frac{1}{r_{n} \mathbb{P}\left(X_{0}>a_{n}\right)}\left(2 m \mathbb{P}\left(X_{0}>a_{n} \epsilon\right)+\left(r_{n}-2 m\right) \mathbb{P}\left(M_{-r_{n},-m-1} \vee M_{m+1, r_{n}}>a_{n} \epsilon, X_{0}>a_{n} \epsilon\right)\right) \\
= & \frac{\mathbb{P}\left(X_{0}>a_{n} \epsilon\right)}{\mathbb{P}\left(X_{0}>a_{n}\right)}\left(\frac{2 m}{r_{n}}+\left(1-\frac{2 m}{r_{n}}\right) \mathbb{P}\left(M_{-r_{n},-m-1} \vee M_{m+1, r_{n}}>a_{n} \epsilon \mid X_{0}>a_{n} \epsilon\right)\right) .
\end{aligned}
$$

Note $\frac{\mathbb{P}\left(X_{0}>a_{n} \epsilon\right)}{\mathbb{P}\left(X_{0}>a_{n}\right)} \sim \epsilon^{-\alpha}$ and $r_{n} \rightarrow \infty$ as $n \rightarrow \infty$, hence, (3.2) implies that

$$
\lim _{m \rightarrow \infty} \limsup _{n \rightarrow \infty} \Delta_{n, m}=0
$$

Observe that on the event $M_{-m,-1} \leq a_{n} \epsilon$, we have $c_{n}(-m, m)=c_{n}(0, m), n \in \mathbb{N}$. Hence,

$$
\frac{\mathbb{E}\left[c_{n}(-m, m) \mathbb{1}_{\left\{M_{-m,-1} \leq a_{n} \epsilon<X_{0}\right\}}\right]}{\mathbb{P}\left(X_{0}>a_{n}\right)}=\frac{\mathbb{P}\left(X_{0}>a_{n} \epsilon\right)}{\mathbb{P}\left(X_{0}>a_{n}\right)} \mathbb{E}\left[c_{n}(0, m) \mathbb{1}_{\left\{M_{-m,-1} \leq a_{n} \epsilon\right\}} \mid X_{0}>a_{n} \epsilon\right]
$$

The first term on the right hand side of (3.11) tends to $\epsilon^{-\alpha}$ as $n \rightarrow \infty$. For each $m \in \mathbb{N}$, we have

$$
c_{n}(0, m)=1-\exp \left\{-\sum_{j=0}^{m} f\left(\frac{X_{j}}{a_{n}}, \frac{M_{j+1}}{\sqrt{X_{j}}}\right)\right\}=1-\exp \left\{-\sum_{j=0}^{m} f\left(\frac{X_{j}}{a_{n}}, W_{j}^{\prime}\right)\right\},
$$

hence, by Proposition 2.1 and a consequence of the conditional continuous mapping theorem (see part (ii) of Lemma D.3) with a bounded Borel measurable function $\widetilde{h}: \mathbb{R}^{3 m+2} \rightarrow \mathbb{R}$ satisfying

$$
\widetilde{h}\left(x_{-m}, \ldots, x_{m}, w_{0}^{\prime}, \ldots, w_{m}^{\prime}\right)=\left(1-\exp \left\{-\sum_{j=0}^{m} f\left(\epsilon x_{j}, w_{j}^{\prime}\right) \mathbb{1}_{(0, \infty)}\left(x_{j}\right)\right\}\right) \mathbb{1}_{\left\{\max \left\{x_{-m}, \ldots, x_{-1}\right\} \leq 1\right\}}
$$

for $x_{-m}, \ldots, x_{m} \in[0, \infty), w_{0}^{\prime}, \ldots, w_{m}^{\prime} \in \mathbb{R}$, we obtain

$$
\begin{aligned}
& \mathbb{E}\left(c_{n}(0, m) \mathbb{1}_{\left\{M_{-m,-1} \leq a_{n} \epsilon\right\}} \mid X_{0}>a_{n} \epsilon\right) \\
& \rightarrow \mathbb{E}\left(\left(1-\exp \left\{-\sum_{j=0}^{m} f\left(\epsilon \mu_{A}^{j} Y_{0}, Z_{j}\right)\right\}\right) \mathbb{1}_{\left\{\max \left\{Y_{-m}, \ldots, Y_{-1}\right\} \leq 1\right\}}\right) \quad \text { as } n \rightarrow \infty
\end{aligned}
$$

since the absolute continuity of $Y_{0}$ and the independence of $Y_{0}$ and $K$ imply

$$
\begin{aligned}
\mathbb{P}\left(\left(Y_{-m}, \ldots, Y_{m}, Z_{0}, \ldots, Z_{m}\right) \in D_{\widetilde{h}}\right) & =\mathbb{P}\left(\max \left\{Y_{-m}, \ldots, Y_{-1}\right\}=1\right) \\
& =\mathbb{P}\left(Y_{0} \max \left\{\mu_{A}^{-m} \mathbb{1}_{\{K \geq m\}}, \ldots, \mu_{A}^{-1} \mathbb{1}_{\{K \geq 1\}}\right\}=1\right)=0,
\end{aligned}
$$


where $D_{\widetilde{h}}$ denotes the set of discontinuities of $\widetilde{h}$. Consequently, by the dominated convergence theorem, the second term on the right hand side of (3.11) as $n \rightarrow \infty$ and then as $m \rightarrow \infty$, converges to

$$
\begin{aligned}
& \mathbb{E}\left[\left(1-\exp \left\{-\sum_{j=0}^{\infty} f\left(\epsilon \mu_{A}^{j} Y_{0}, Z_{j}\right)\right\}\right) \mathbb{1}_{\left\{\sup _{j<0} Y_{j} \leq 1\right\}}\right] \\
& =\mathbb{E}\left[\left(1-\exp \left\{-\sum_{j=0}^{\infty} f\left(\epsilon \mu_{A}^{j} Y_{0}, Z_{j}\right)\right\}\right) \mathbb{1}_{\{K=0\}}\right]=\theta \mathbb{E}\left[1-\exp \left\{-\sum_{j=0}^{\infty} f\left(\epsilon \mu_{A}^{j} Y_{0}, Z_{j}\right)\right\}\right]
\end{aligned}
$$

where the last step follows by the fact that $K$ is independent of $Y_{0}$ and $\left(Z_{j}\right)_{j \geq 0}$ and since $\mathbb{P}(K=0)=\theta$ (see (3.4)). Altogether, using the fact that $Y_{0}$ is Pareto distributed such that $\mathbb{P}\left(Y_{0} \geq y\right)=y^{-\alpha}, y \geq 1$, and independent of $\left(Z_{j}\right)_{j \geq 0}$,

$$
\begin{aligned}
\lim _{n \rightarrow \infty} \frac{\mathbb{E}\left[c_{n}\left(1, r_{n}\right)\right]}{r_{n} \mathbb{P}\left(X_{0}>a_{n}\right)} & =\lim _{m \rightarrow \infty} \lim _{n \rightarrow \infty} \frac{\mathbb{E}\left[c_{n}(-m, m) \mathbb{1}_{\left\{M_{-m,-1} \leq a_{n} \epsilon<X_{0}\right\}}\right]}{\mathbb{P}\left(X_{0}>a_{n}\right)} \\
& =\epsilon^{-\alpha} \theta \int_{1}^{\infty} \mathbb{E}\left[1-\exp \left\{-\sum_{j=0}^{\infty} f\left(\epsilon y \mu_{A}^{j}, Z_{j}\right)\right\}\right] \alpha y^{-\alpha-1} \mathrm{~d} y \\
& =\theta \int_{\epsilon}^{\infty} \mathbb{E}\left[1-\exp \left\{-\sum_{j=0}^{\infty} f\left(y \mu_{A}^{j}, Z_{j}\right)\right\}\right] \alpha y^{-\alpha-1} \mathrm{~d} y \\
& =\theta \int_{0}^{\infty} \mathbb{E}\left[1-\exp \left\{-\sum_{j=0}^{\infty} f\left(y \mu_{A}^{j}, Z_{j}\right)\right\}\right] \alpha y^{-\alpha-1} \mathrm{~d} y,
\end{aligned}
$$

where the last line follows since $f(x, y)=0$ if $x \leq \epsilon, y \in \mathbb{R}$, and $\mu_{A} \in(0,1)$, and this concludes the proof of (ii) yielding the statement.

\section{From point processes to sums}

The key idea in handling the sums $\sum_{j=1}^{n} X_{j}^{2}$ and $\sum_{j=1}^{n} X_{j} M_{j+1}$ (the building blocks of ${\widehat{\mu_{A}}}^{(n)}-$ $\left.\mu_{A}\right)$ is to apply usual truncation argument and then summation to obtain the following result, cf. Davis and Hsing [11, Theorem 3.1].

Theorem 4.1. We have

$$
\left(V_{n}^{(1)}, V_{n}^{(2)}\right):=\left(\frac{1}{a_{n}^{2}} \sum_{j=1}^{n} X_{j}^{2}, \frac{1}{a_{n}^{3 / 2}} \sum_{j=1}^{n} X_{j} M_{j+1}\right) \stackrel{d}{\longrightarrow}\left(V^{(1)}, V^{(2)}\right)
$$

as $n \rightarrow \infty$ with

$$
\left(V^{(1)}, V^{(2)}\right) \stackrel{d}{=}\left(\frac{1}{1-\mu_{A}^{2}} \sum_{i=1}^{\infty} P_{i}^{2}, \frac{1}{\left(1-\mu_{A}^{3}\right)^{1 / 2}} \sum_{i=1}^{\infty} P_{i}^{3 / 2} Z_{i}\right)
$$


where $\sum_{i=1}^{\infty} \delta_{P_{i}}$ is a Poisson point process on $(0, \infty)$ with intensity $\theta \mathrm{d}\left(-y^{-\alpha}\right)$ such that $P_{1} \geq$ $P_{2} \geq \ldots$ almost surely, $\left(Z_{i}\right)_{i \geq 0}$ is an i.i.d. sequence of $\mathcal{N}\left(0, \sigma_{A}^{2}\right)$-distributed random variables independent of $\sum_{i=1}^{\infty} \delta_{P_{i}}$ with $\theta$ given in (3.4), and the series on the right hand side of (4.2) are convergent almost surely.

The characteristic function of the vector $\left(V^{(1)}, V^{(2)}\right)$ has the form

$$
\mathbb{E}\left[\mathrm{e}^{\mathrm{i}\left(s V^{(1)}+t V^{(2)}\right)}\right]=\exp \left\{\theta \int_{0}^{\infty}\left(\exp \left\{\frac{\mathrm{i} s}{1-\mu_{A}^{2}} y^{2}-\frac{\sigma_{A}^{2} t^{2}}{2\left(1-\mu_{A}^{3}\right)} y^{3}\right\}-1\right) \alpha y^{-\alpha-1} \mathrm{~d} y\right\}
$$

for $s, t \in \mathbb{R}$. For the marginals, we have

$$
\mathbb{E}\left[\mathrm{e}^{\mathrm{i} s V^{(1)}}\right]=\exp \left\{-C_{1}|s|^{\alpha / 2}\left(1-\mathrm{i} \tan \left(\frac{\pi \alpha}{4}\right) \operatorname{sgn}(s)\right)\right\}, \quad \mathbb{E}\left[\mathrm{e}^{\mathrm{i} t V^{(2)}}\right]=\mathrm{e}^{-C_{2}|t|^{2 \alpha / 3}}
$$

for $s, t \in \mathbb{R}$ with

$$
C_{1}:=\theta \Gamma\left(1-\frac{\alpha}{2}\right) \frac{\cos \left(\frac{\pi \alpha}{4}\right)}{\left(1-\mu_{A}^{2}\right)^{\alpha / 2}}, \quad C_{2}:=\theta \Gamma\left(1-\frac{\alpha}{3}\right)\left(\frac{\sigma_{A}^{2}}{2\left(1-\mu_{A}^{3}\right)}\right)^{\alpha / 3}
$$

thus $V^{(1)}$ is an $\alpha / 2$-stable positive random variable and $V^{(2)}$ is a symmetric $2 \alpha / 3$-stable random variable.

Proof. We divide the proof into four steps.

Step 1. Applying continuous mapping theorem to the convergence in (3.5) we show that

$$
\begin{aligned}
& \left(V_{n, \gamma}^{(1)}, V_{n, \gamma}^{(2)}\right):=\left(\sum_{j=1}^{n}\left(X_{j} / a_{n}\right)^{2} \mathbb{1}_{\left\{X_{j} / a_{n}>\gamma\right\}}, \sum_{j=1}^{n} X_{j} M_{j+1} / a_{n}^{3 / 2} \mathbb{1}_{\left\{X_{j} / a_{n}>\gamma\right\}}\right) \\
& \stackrel{d}{\longrightarrow}\left(V_{\gamma}^{(1)}, V_{\gamma}^{(2)}\right):=\left(\sum_{i=1}^{\infty} \sum_{j=0}^{\infty}\left(P_{i} \mu_{A}^{j}\right)^{2} \mathbb{1}_{\left\{P_{i} \mu_{A}^{j}>\gamma\right\}}, \sum_{i=1}^{\infty} \sum_{j=0}^{\infty}\left(P_{i} \mu_{A}^{j}\right)^{3 / 2} Z_{i, j} \mathbb{1}_{\left\{P_{i} \mu_{A}^{j}>\gamma\right\}}\right)
\end{aligned}
$$

as $n \rightarrow \infty$ for all $\gamma>0$, where $Z_{i, j}, i \in \mathbb{N}, j \geq 0$, are given in Theorem 3.2. Fix $\gamma>0$, and consider the mapping $T_{\gamma}: \mathcal{M}_{p}(S) \rightarrow \mathbb{R}^{2}$,

$$
\begin{aligned}
T_{\gamma}(\kappa) & :=\left(\int_{S} x^{2} \mathbb{1}_{\{x>\gamma\}} \kappa(\mathrm{d} x, \mathrm{~d} y), \int_{S} x^{3 / 2} y \mathbb{1}_{\{x>\gamma\}} \kappa(\mathrm{d} x, \mathrm{~d} y)\right) \\
& =\left(\sum_{k} x_{k}^{2} \mathbb{1}_{\left\{x_{k}>\gamma\right\}}, \sum_{k} x_{k}^{3 / 2} y_{k} \mathbb{1}_{\left\{x_{k}>\gamma\right\}}\right)
\end{aligned}
$$

for $\kappa=\sum_{k} \delta_{\left(x_{k}, y_{k}\right)} \in \mathcal{M}_{p}(S)$. Note that the sums in the definition of $T_{\gamma}(\kappa)$ are sums with finitely many terms, since the set $(\gamma, \infty) \times \mathbb{R}$ is bounded and $\kappa$ is a locally finite measure on $S$. By Lemma 3.1, $T_{\gamma}$ is continuous on $C_{\gamma}:=\left\{\kappa \in \mathcal{M}_{p}(S): \kappa(\{\gamma\} \times \mathbb{R})=0\right\}$, i.e., for any $\kappa \in C_{\gamma}$ and any sequence $\left(\kappa_{n}\right)_{n \in \mathbb{N}}$ in $\mathcal{M}_{p}(S)$ such that $\kappa_{n} \stackrel{v}{\rightarrow} \kappa$ as $n \rightarrow \infty$, we have $T_{\gamma}\left(\kappa_{n}\right) \rightarrow T_{\gamma}(\kappa)$ as 
$n \rightarrow \infty$. Indeed, by Lemma 3.1, $\kappa_{n} \stackrel{v}{\rightarrow} \kappa$ as $n \rightarrow \infty$ yields that there exist integers $n_{0}, M \geq 0$ and a labeling of the points of $\kappa$ and $\kappa_{n}, n \geq n_{0}$, in $(\gamma, \infty) \times \mathbb{R}$ such that

$$
\left.\kappa_{n}\right|_{(\gamma, \infty) \times \mathbb{R}}=\sum_{k=1}^{M} \delta_{\left(x_{k}^{(n)}, y_{k}^{(n)}\right)}, \quad n \geq n_{0},\left.\quad \kappa\right|_{(\gamma, \infty) \times \mathbb{R}}=\sum_{k=1}^{M} \delta_{\left(x_{k}, y_{k}\right)},
$$

and $\left(x_{k}^{(n)}, y_{k}^{(n)}\right) \rightarrow\left(x_{k}, y_{k}\right)$ as $n \rightarrow \infty$ for all $k=1, \ldots, M$. Hence

$$
\begin{aligned}
& T_{\gamma}\left(\kappa_{n}\right)=\left(\sum_{k=1}^{M}\left(x_{k}^{(n)}\right)^{2}, \sum_{k=1}^{M}\left(x_{k}^{(n)}\right)^{3 / 2} y_{k}^{(n)}\right), \quad n \geq n_{0}, \\
& T_{\gamma}(\kappa)=\left(\sum_{k=1}^{M} x_{k}^{2}, \sum_{k=1}^{M} x_{k}^{3 / 2} y_{k}\right),
\end{aligned}
$$

yielding that $T_{\gamma}\left(\kappa_{n}\right) \rightarrow T_{\gamma}(\kappa)$ as $n \rightarrow \infty$, as desired. Finally, $\left(V_{n, \gamma}^{(1)}, V_{n, \gamma}^{(2)}\right) \stackrel{d}{\longrightarrow}\left(V_{\gamma}^{(1)}, V_{\gamma}^{(2)}\right)$ as $n \rightarrow \infty$ follows by an application of the continuous mapping theorem (see, e.g., Resnick [36]). Indeed, $\mathcal{M}_{p}(S)$ is a complete separable metric space with a metric inducing the vague topology (see Kallenberg [21, Lemma 4.6]), $\left(V_{n, \gamma}^{(1)}, V_{n, \gamma}^{(2)}\right)=T_{\gamma}\left(N_{n}\right), n \in \mathbb{N}, T_{\gamma}(N)=\left(V_{\gamma}^{(1)}, V_{\gamma}^{(2)}\right)$, and we check that $\mathbb{P}\left(N \in C_{\gamma}\right)=1$. At the beginning of the proof of Theorem 3.2, we already checked that $N$ is a locally finite measure almost surely, so it remains to verify that $\mathbb{P}(N(\{\gamma\} \times \mathbb{R})=$ $0)=1$. We have

$$
N(\{\gamma\} \times \mathbb{R})=\sum_{i=1}^{\infty} \sum_{j=0}^{\infty} \mathbb{1}_{\left\{P_{i} \mu_{A}^{j}=\gamma\right\}}=\left(\sum_{i=1}^{\infty} \delta_{P_{i}}\right)\left(\left\{\gamma, \mu_{A}^{-1} \gamma, \mu_{A}^{-2} \gamma, \ldots\right\}\right)=0 \text { a.s. }
$$

since the intensity measure of the point process $\sum_{i=1}^{\infty} \delta_{P_{i}}$ is absolutely continuous.

Step 2. We check that $\left(V_{\gamma}^{(1)}, V_{\gamma}^{(2)}\right) \stackrel{\mathbb{P}}{\longrightarrow}\left(V^{(1)}, V^{(2)}\right)$ as $\gamma \downarrow 0$, where

$$
V^{(1)}:=\left(1-\mu_{A}^{2}\right)^{-1} \sum_{i=1}^{\infty} P_{i}^{2}, \quad V^{(2)}:=\left(1-\mu_{A}^{3}\right)^{-1 / 2} \sum_{i=1}^{\infty} P_{i}^{3 / 2} Z_{i}
$$

with

$$
Z_{i}:=\left(1-\mu_{A}^{3}\right)^{1 / 2} \sum_{j=0}^{\infty}\left(\mu_{A}^{j}\right)^{3 / 2} Z_{i, j}, \quad i \in \mathbb{N}
$$

where $\left(P_{i}\right)_{i \in \mathbb{N}}$ and $\left\{Z_{i, j}: i \in \mathbb{N}, j \geq 0\right\}$ are given in Theorem 3.2.

By monotonicity of $V_{\gamma}^{(1)}$ in $\gamma>0$,

$$
V_{\gamma}^{(1)} \rightarrow\left(1-\mu_{A}^{2}\right)^{-1} \sum_{i=1}^{\infty} P_{i}^{2}=V^{(1)} \quad \text { as } \gamma \downarrow 0
$$

almost surely. By Campbell's theorem (see, e.g., Kingman [23, Section 3.2]), we have $\mathbb{P}\left(\sum_{i=1}^{\infty} P_{i}^{\beta}<\right.$ $\infty)=1$ for any $\beta \in(\alpha, \infty)$, and hence the series $\sum_{i=1}^{\infty} P_{i}^{2}$ is (absolutely) convergent almost 
surely, since $\alpha \in(1,2)$. Indeed, condition (3.16) in Kingman [23] is satisfied, since

$$
\begin{aligned}
\int_{0}^{\infty}\left(y^{\beta} \wedge 1\right) \theta \mathrm{d}\left(-y^{-\alpha}\right) & =\theta \alpha \int_{0}^{\infty}\left(y^{\beta} \wedge 1\right) y^{-\alpha-1} \mathrm{~d} y=\theta \alpha \int_{0}^{1} y^{\beta-\alpha-1} \mathrm{~d} y+\theta \alpha \int_{1}^{\infty} y^{-\alpha-1} \mathrm{~d} y \\
& =\theta \alpha\left(\frac{1}{\beta-\alpha}+\frac{1}{\alpha}\right)<\infty
\end{aligned}
$$

Next, we show that the series $\sum_{j=0}^{\infty}\left(\mu_{A}^{j}\right)^{3 / 2} Z_{i, j}, i \in \mathbb{N}$, and $\sum_{i=1}^{\infty} P_{i}^{3 / 2} Z_{i}$ are convergent almost surely. Kolmogorov's one series theorem yields that $\sum_{j=0}^{\infty}\left(\mu_{A}^{j}\right)^{3 / 2} Z_{i, j}$ converges almost surely for each $i \in \mathbb{N}$, and hence $\left(Z_{i}\right)_{i \in \mathbb{N}}$ is an i.i.d. sequence of $\mathcal{N}\left(0, \sigma_{A}^{2}\right)$-distributed random variables independent of $\left(P_{i}\right)_{i \in \mathbb{N}}$. Let $E_{j}, j \in \mathbb{N}$, be i.i.d. random variables with an exponential distribution with parameter 1 , independent of $Z_{i}, i \in \mathbb{N}$. Put $\Gamma_{i}:=\sum_{j=1}^{i} E_{j}, i \in \mathbb{N}$. By the mapping theorem for Poisson random measures, $\sum_{i=1}^{\infty} \delta_{\theta^{1 / \alpha} \Gamma_{i}^{-1 / \alpha}}$ is a Poisson random measure on $(0, \infty)$ with intensity $\theta \mathrm{d}\left(-y^{-\alpha}\right)$, hence we have $\left(P_{i}\right)_{i \in \mathbb{N}} \stackrel{d}{=}\left(\theta^{1 / \alpha} \Gamma_{i}^{-1 / \alpha}\right)_{i \in \mathbb{N}}$, and hence, $\left(P_{i}, Z_{i}\right)_{i \in \mathbb{N}} \stackrel{d}{=}\left(\theta^{1 / \alpha} \Gamma_{i}^{-1 / \alpha}, Z_{i}\right)_{i \in \mathbb{N}}$. Consequently,

$$
\left(\sum_{i=1}^{n} P_{i}^{3 / 2} Z_{i}\right)_{n \in \mathbb{N}} \stackrel{d}{=}\left(\sum_{i=1}^{n}\left(\theta^{1 / \alpha} \Gamma_{i}^{-1 / \alpha}\right)^{3 / 2} Z_{i}\right)_{n \in \mathbb{N}}=\left(\theta^{3 /(2 \alpha)} \sum_{i=1}^{n} \Gamma_{i}^{-3 /(2 \alpha)} Z_{i}\right)_{n \in \mathbb{N}},
$$

thus the almost sure convergence of $\sum_{i=1}^{\infty} P_{i}^{3 / 2} Z_{i}$ will follow from the almost sure convergence of $\sum_{i=1}^{\infty} \Gamma_{i}^{-3 /(2 \alpha)} Z_{i}$. Indeed, if $\sum_{i=1}^{\infty} \Gamma_{i}^{-3 /(2 \alpha)} Z_{i}$ is convergent almost surely, then for each $\epsilon \in$ $(0, \infty)$, by the continuity of probability, we have

$$
\mathbb{P}\left[\sup _{m \in \mathbb{N}}\left|\sum_{i=n}^{n+m} P_{i}^{3 / 2} Z_{i}\right|>\epsilon\right]=\mathbb{P}\left[\theta^{3 /(2 \alpha)} \sup _{m \in \mathbb{N}}\left|\sum_{i=n}^{n+m} \Gamma_{i}^{-3 /(2 \alpha)} Z_{i}\right|>\epsilon\right] \rightarrow 0 \quad \text { as } n \rightarrow \infty
$$

implying the almost sure convergence of $\sum_{i=1}^{\infty} P_{i}^{3 / 2} Z_{i}$, see, e.g., Shiryaev [41, Chapter II, Section 3, Theorem 1]. The almost sure convergence of $\sum_{i=1}^{\infty} \Gamma_{i}^{-3 /(2 \alpha)} Z_{i}$ follows from Theorem 1.4.5 in Samorodnitsky and Taqqu [37], since $\frac{2 \alpha}{3} \in(0,2), \mathbb{E}\left[\left|Z_{1}\right|^{2 \alpha / 3}\right]<\infty, \mathbb{E}\left[\left|Z_{1} \log \left(\left|Z_{1}\right|\right)\right|\right]<\infty$, $\mathbb{E}\left[Z_{1}\right]=0$ and $\mathbb{E}\left[Z_{1} \int_{\left|Z_{1}\right| / i}^{\left|Z_{1}\right| /(i-1)} x^{-2} \sin (x) \mathrm{d} x\right]=0$ for all $i \in \mathbb{N}$, where $\left|Z_{1}\right| /(i-1):=\infty$ for $i=1$ (due to the fact that $Z_{1}$ is symmetric). Hence the series $\sum_{i=1}^{\infty} P_{i}^{3 / 2} Z_{i}$ converges almost surely and $V^{(2)}$ is well--defined.

Next, we show that $V_{\gamma}^{(2)} \stackrel{\mathbb{P}}{\longrightarrow} V^{(2)}$ as $\gamma \downarrow 0$. For every $\gamma, \epsilon \in(0, \infty)$ define

$$
V_{\gamma, \epsilon}^{(2)}:=\sum_{i=1}^{\infty} \sum_{j=0}^{\infty}\left(P_{i} \mu_{A}^{j}\right)^{3 / 2} Z_{i, j} \mathbb{1}_{\left\{P_{i}>\epsilon, P_{i} \mu_{A}^{j}>\gamma\right\}} .
$$

Since for every $\epsilon \in(0, \infty)$ there are almost surely only finitely many $P_{i}$ 's greater that $\epsilon$, for every fixed $\epsilon \in(0, \infty)$,

$$
V_{\gamma, \epsilon}^{(2)} \rightarrow V_{0, \epsilon}^{(2)}:=\left(1-\mu_{A}^{3}\right)^{-1 / 2} \sum_{i=1}^{\infty} P_{i}^{3 / 2} Z_{i} \mathbb{1}_{\left\{P_{i}>\epsilon\right\}} \quad \text { as } \gamma \downarrow 0
$$


almost surely. Indeed, by the dominated convergence theorem, for every $i \in \mathbb{N}$, we have $\sum_{j=0}^{\infty} \mu_{A}^{3 j / 2} Z_{i, j} \mathbb{1}_{\left\{P_{i} \mu_{A}^{j}>\gamma\right\}} \rightarrow\left(1-\mu_{A}^{3}\right)^{-1 / 2} Z_{i}$ as $\gamma \downarrow 0$ almost surely, since $\left|\mu_{A}^{3 j / 2} Z_{i, j} \mathbb{1}_{\left\{P_{i} \mu_{A}^{j}>\gamma\right\}}\right| \leq$ $\mu_{A}^{3 j / 2}\left|Z_{i, j}\right|, j \geq 0$, yielding that

$$
\left|\sum_{j=0}^{\infty} \mu_{A}^{3 j / 2} Z_{i, j} \mathbb{1}_{\left\{P_{i} \mu_{A}^{j}>\gamma\right\}}\right| \leq \sum_{j=0}^{\infty} \mu_{A}^{3 j / 2}\left|Z_{i, j}\right|,
$$

where $\mathbb{E}\left[\sum_{j=0}^{\infty} \mu_{A}^{3 j / 2}\left|Z_{i, j}\right|\right]=\mathbb{E}\left[\left|Z_{1,1}\right|\right] /\left(1-\mu_{A}^{3 / 2}\right)<\infty$ (especially, $\sum_{j=0}^{\infty} \mu_{A}^{3 j / 2}\left|Z_{i, j}\right|$ converges almost surely).

Now we check that $V_{0, \epsilon}^{(2)} \rightarrow V^{(2)}$ as $\epsilon \downarrow 0$ almost surely. For each $\epsilon \in(0, \infty)$, we can write

$$
V_{0, \epsilon}^{(2)}=\left(1-\mu_{A}^{3}\right)^{-1 / 2} \sum_{i=1}^{K_{\epsilon}} P_{i}^{3 / 2} Z_{i}
$$

with $K_{\epsilon}:=\max \left\{i \in \mathbb{N}: P_{i}>\epsilon\right\}$. We have $K_{\epsilon} \rightarrow \infty$ as $\epsilon \downarrow 0$ almost surely, since $P_{i} \downarrow 0$ as $i \rightarrow \infty$ almost surely, thus the almost sure convergence of $\sum_{i=1}^{\infty} P_{i}^{3 / 2} Z_{i}$ yields that $V_{0, \epsilon}^{(2)} \rightarrow V^{(2)}$ as $\epsilon \downarrow 0$ almost surely.

For every $\gamma, \epsilon, \eta \in(0, \infty)$, we have $\mathbb{P}\left(\left|V_{\gamma}^{(2)}-V^{(2)}\right|>\eta\right) \leq \mathbb{P}\left(\left|V_{\gamma}^{(2)}-V_{\gamma, \epsilon}^{(2)}\right|>\eta / 3\right)+\mathbb{P}\left(\left|V_{\gamma, \epsilon}^{(2)}-V_{0, \epsilon}^{(2)}\right|>\eta / 3\right)+\mathbb{P}\left(\left|V_{0, \epsilon}^{(2)}-V^{(2)}\right|>\eta / 3\right)$. The almost sure convergences $V_{\gamma, \epsilon}^{(2)} \stackrel{\text { a.s. }}{\longrightarrow} V_{0, \epsilon}^{(2)}$ as $\gamma \downarrow 0$ for all $\epsilon \in(0, \infty)$ and $V_{0, \epsilon}^{(2)} \stackrel{\text { a.s. }}{\longrightarrow} V^{(2)}$ as $\epsilon \downarrow 0$ imply the corresponding convergences in probability, hence

$$
\limsup _{\gamma \downarrow 0} \mathbb{P}\left(\left|V_{\gamma}^{(2)}-V^{(2)}\right|>\eta\right) \leq \limsup _{\gamma \downarrow 0} \mathbb{P}\left(\left|V_{\gamma}^{(2)}-V_{\gamma, \epsilon}^{(2)}\right|>\eta / 3\right)+\mathbb{P}\left(\left|V_{0, \epsilon}^{(2)}-V^{(2)}\right|>\eta / 3\right)
$$

for every $\epsilon, \eta \in(0, \infty)$, and hence

$$
\limsup _{\gamma \downarrow 0} \mathbb{P}\left(\left|V_{\gamma}^{(2)}-V^{(2)}\right|>\eta\right) \leq \limsup _{\epsilon \downarrow 0} \limsup _{\gamma \downarrow 0} \mathbb{P}\left(\left|V_{\gamma, \epsilon}^{(2)}-V_{\gamma}^{(2)}\right|>\eta / 3\right)
$$

for every $\eta \in(0, \infty)$. Consequently, if we show that for all $\eta \in(0, \infty)$,

$$
\limsup _{\epsilon \downarrow 0} \limsup _{\gamma \downarrow 0} \mathbb{P}\left(\left|V_{\gamma, \epsilon}^{(2)}-V_{\gamma}^{(2)}\right|>\eta\right)=0,
$$

then we obtain $V_{\gamma}^{(2)} \stackrel{\mathbb{P}}{\longrightarrow} V^{(2)}$ as $\gamma \downarrow 0$, as desired. In order to check (4.3), observe

$$
V_{\gamma}^{(2)}-V_{\gamma, \epsilon}^{(2)}=\sum_{i=1}^{\infty} \sum_{j=0}^{\infty}\left(P_{i} \mu_{A}^{j}\right)^{3 / 2} Z_{i, j} \mathbb{1}_{\left\{P_{i} \leq \epsilon, P_{i} \mu_{A}^{j}>\gamma\right\}},
$$

since the sums defining $V_{\gamma, \epsilon}^{(2)}$ and $V_{\gamma}^{(2)}$ are sums with finitely many terms almost surely (see Step 
1). Since $\mathbb{E}\left[V_{\gamma, \epsilon}^{(2)}-V_{\gamma}^{(2)}\right]=0$ for all $\gamma, \epsilon \in(0, \infty)$, we have

$$
\begin{aligned}
\operatorname{Var}\left(V_{\gamma, \epsilon}^{(2)}-V_{\gamma}^{(2)}\right) & =\mathbb{E}\left[\left(V_{\gamma, \epsilon}^{(2)}-V_{\gamma}^{(2)}\right)^{2}\right]=\mathbb{E}\left[\mathbb{E}\left[\left(V_{\gamma, \epsilon}^{(2)}-V_{\gamma}^{(2)}\right)^{2} \mid\left(P_{i}\right)_{i \in \mathbb{N}}\right]\right] \\
& =\mathbb{E}\left[\sum_{i=1}^{\infty} \sum_{j=0}^{\infty}\left(P_{i} \mu_{A}^{j}\right)^{3} \sigma_{A}^{2} \mathbb{1}_{\left\{P_{i} \leq \epsilon, P_{i} \mu_{A}^{j}>\gamma\right\}}\right] \leq \mathbb{E}\left[\sum_{i=1}^{\infty} \sum_{j=0}^{\infty}\left(P_{i} \mu_{A}^{j}\right)^{3} \sigma_{A}^{2} \mathbb{1}_{\left\{P_{i} \leq \epsilon\right\}}\right] \\
& \leq \frac{\sigma_{A}^{2}}{1-\mu_{A}^{3}} \mathbb{E}\left[\sum_{i=1}^{\infty} P_{i}^{3} \mathbb{1}_{\left\{P_{i} \leq \epsilon\right\}}\right]=\frac{\theta \sigma_{A}^{2}}{1-\mu_{A}^{3}} \int_{0}^{\epsilon} x^{3} \alpha x^{-\alpha-1} \mathrm{~d} x=\frac{\theta \sigma_{A}^{2} \alpha \epsilon^{3-\alpha}}{\left(1-\mu_{A}^{3}\right)(3-\alpha)},
\end{aligned}
$$

where the last but one step follows by Campbell's theorem (see, e.g., Kingman [23, Section $3.2])$. For all $\eta \in(0, \infty)$, Chebyshev's inequality implies that

$$
\limsup _{\epsilon \downarrow 0} \limsup _{\gamma \downarrow 0} \mathbb{P}\left(\left|V_{\gamma, \epsilon}^{(2)}-V_{\gamma}^{(2)}\right|>\eta\right) \leq \limsup _{\epsilon \downarrow 0} \frac{\theta \sigma_{A}^{2} \alpha \epsilon^{3-\alpha}}{\eta^{2}\left(1-\mu_{A}^{3}\right)(3-\alpha)}=0,
$$

hence we conclude (4.3), as desired. Altogether,

$$
\left(V_{\gamma}^{(1)}, V_{\gamma}^{(2)}\right) \stackrel{\mathbb{P}}{\longrightarrow}\left(V^{(1)}, V^{(2)}\right) \quad \text { as } \gamma \downarrow 0,
$$

and hence in distribution as well.

Step 3. By Billingsley [8, Theorem 4.2] and Steps 1 and 2, using also that $\left\|\left(z_{1}, z_{2}\right)\right\| \leq$ $\left|z_{1}\right|+\left|z_{2}\right|,\left(z_{1}, z_{2}\right) \in \mathbb{R}^{2}$, to show (4.1), it suffices to prove that for all $\epsilon>0$,

$$
\lim _{\gamma \downarrow 0} \limsup _{n \rightarrow \infty} \mathbb{P}\left(\left|V_{n}^{(k)}-V_{n, \gamma}^{(k)}\right|>\epsilon\right)=0, \quad k=1,2 .
$$

In case of $k=1$, by Markov's inequality, (3.1) and Karamata's theorem (see, Lemma E.1), we have

$$
\begin{aligned}
\lim _{\gamma \downarrow 0} \limsup _{n \rightarrow \infty} \mathbb{P}\left(\left|V_{n}^{(1)}-V_{n, \gamma}^{(1)}\right|>\epsilon\right) & =\lim _{\gamma \downarrow 0} \limsup _{n \rightarrow \infty} \mathbb{P}\left(\sum_{j=1}^{n} X_{j}^{2} \mathbb{1}_{\left\{X_{j} / a_{n} \leq \gamma\right\}}>a_{n}^{2} \epsilon\right) \\
& \leq \lim _{\gamma \downarrow 0} \limsup _{n \rightarrow \infty} \frac{\gamma^{2}}{\epsilon} \frac{\mathbb{E}\left[X_{1}^{2} \mathbb{1}_{\left\{X_{1} \leq a_{n} \gamma\right\}}\right]}{\gamma^{2} a_{n}^{2} \mathbb{P}\left(X_{1}>a_{n} \gamma\right)} \frac{\mathbb{P}\left(X_{1}>a_{n} \gamma\right)}{\mathbb{P}\left(X_{1}>a_{n}\right)} n \mathbb{P}\left(X_{1}>a_{n}\right) \\
& =\lim _{\gamma \downarrow 0} \frac{\alpha}{\epsilon(2-\alpha)} \gamma^{2-\alpha}=0,
\end{aligned}
$$

as desired. In case of $k=2$, we have to prove that

$$
\lim _{\gamma \downarrow 0} \limsup _{n \rightarrow \infty} \mathbb{P}\left(\left|\sum_{j=1}^{n} X_{j} M_{j+1} \mathbb{1}_{\left\{X_{j} / a_{n} \leq \gamma\right\}}\right|>a_{n}^{3 / 2} \epsilon\right)=0
$$

for all $\epsilon>0$. Using the definition of $M_{j+1}$, we have

$$
\begin{aligned}
\mathbb{P}\left(\left|\sum_{j=1}^{n} X_{j} M_{j+1} \mathbb{1}_{\left\{X_{j} / a_{n} \leq \gamma\right\}}\right|>a_{n}^{3 / 2} \epsilon\right) \leq & \mathbb{P}\left(\left|\sum_{j=1}^{n} X_{j} \sum_{i=1}^{X_{j}} \tilde{A}_{i}^{(j+1)} \mathbb{1}_{\left\{X_{j} / a_{n} \leq \gamma\right\}}\right|>a_{n}^{3 / 2} \epsilon / 2\right) \\
& +\mathbb{P}\left(\left|\sum_{j=1}^{n} X_{j} \mathbb{1}_{\left\{X_{j} / a_{n} \leq \gamma\right\}} \tilde{B}_{j+1}\right|>a_{n}^{3 / 2} \epsilon / 2\right) .
\end{aligned}
$$


Recall that for each $j=1, \ldots, n,\left\{\tilde{A}_{i}^{(j+1)}, i \in \mathbb{N}\right\}$ are i.i.d. random variables with $\mathbb{E}\left[\tilde{A}_{1}^{(j+1)}\right]=0$ and $\operatorname{Var}\left[\tilde{A}_{1}^{(j+1)}\right]=\sigma_{A}^{2}$, and independent of $\left\{\tilde{A}_{i}^{(k)}, i \in \mathbb{N}, k=2, \ldots, j\right\}$ and $X_{1}, \ldots, X_{j}$. This implies that the random variables $X_{j} \sum_{i=1}^{X_{j}} \tilde{A}_{i}^{(j+1)} \mathbb{1}_{\left\{X_{j} / a_{n} \leq \gamma\right\}}, j \in\{1, \ldots, n\}$, are uncorrelated and have zero expectation. Hence, using Markov's inequality and the law of total variance, we get for all $\epsilon>0$,

$$
\begin{aligned}
& \mathbb{P}\left(\left|\sum_{j=1}^{n} X_{j} \sum_{i=1}^{X_{j}} \tilde{A}_{i}^{(j+1)} \mathbb{1}_{\left\{X_{j} / a_{n} \leq \gamma\right\}}\right|>a_{n}^{3 / 2} \epsilon\right) \leq \frac{1}{a_{n}^{3} \epsilon^{2}} \mathbb{E}\left[\left(\sum_{j=1}^{n} X_{j} \sum_{i=1}^{X_{j}} \tilde{A}_{i}^{(j+1)} \mathbb{1}_{\left\{X_{j} / a_{n} \leq \gamma\right\}}\right)^{2}\right] \\
& =\frac{1}{a_{n}^{3} \epsilon^{2}} \mathbb{E}\left[\sum_{j=1}^{n} X_{j}^{2}\left(\sum_{i=1}^{X_{j}} \tilde{A}_{i}^{(j+1)}\right)^{2} \mathbb{1}_{\left\{X_{j} / a_{n} \leq \gamma\right\}}\right]=\frac{n}{a_{n}^{3} \epsilon^{2}} \mathbb{E}\left[X_{0}^{2}\left(\sum_{i=1}^{X_{0}} \tilde{A}_{i}^{(1)}\right)^{2} \mathbb{1}_{\left\{X_{0} / a_{n} \leq \gamma\right\}}\right] \\
& =\frac{n \operatorname{Var}\left[X_{0}\left(\sum_{i=1}^{X_{0}} \tilde{A}_{i}^{(1)}\right) \mathbb{1}_{\left\{X_{0} / a_{n} \leq \gamma\right\}}\right]}{a_{n}^{3} \epsilon^{2}}=\frac{n \sigma_{A}^{2} \mathbb{E}\left[X_{0}^{3} \mathbb{1}_{\left\{X_{0} \leq a_{n} \gamma\right\}}\right.}{a_{n}^{3} \epsilon^{2}} .
\end{aligned}
$$

By Karamata's theorem (see, Lemma E.1) and (3.1), since $\alpha<3$,

$$
\begin{aligned}
\frac{n \sigma_{A}^{2} \mathbb{E}\left[X_{0}^{3} \mathbb{1}_{\left\{X_{0} \leq a_{n} \gamma\right\}}\right]}{a_{n}^{3} \epsilon^{2}} & =\sigma_{A}^{2} \epsilon^{-2} \frac{\mathbb{E}\left[X_{0}^{3} \mathbb{1}_{\left\{X_{0} \leq a_{n} \gamma\right\}}\right]}{a_{n}^{3} \gamma^{3} \mathbb{P}\left(X_{0}>a_{n} \gamma\right)} \gamma^{3} \frac{\mathbb{P}\left(X_{0}>a_{n} \gamma\right)}{\mathbb{P}\left(X_{0}>a_{n}\right)} n \mathbb{P}\left(X_{0}>a_{n}\right) \\
& \rightarrow \sigma_{A}^{2} \epsilon^{-2} \frac{\alpha}{3-\alpha} \gamma^{3-\alpha} \quad \text { as } n \rightarrow \infty
\end{aligned}
$$

which further goes to 0 as $\gamma \downarrow 0$. Hence, (4.4) will follow if we show that

$$
\lim _{\gamma \downarrow 0} \limsup _{n \rightarrow \infty} \mathbb{P}\left(\left|\sum_{j=1}^{n} X_{j} \mathbb{1}_{\left\{X_{j} / a_{n} \leq \gamma\right\}} \tilde{B}_{j+1}\right|>a_{n}^{3 / 2} \epsilon\right)=0
$$

for all $\epsilon>0$. With the notation $c_{B, n}:=\mathbb{E}\left[\tilde{B}_{j+1} \mathbb{1}_{\left\{\left|\tilde{B}_{j+1}\right| / a_{n} \leq 1\right\}}\right]=\mathbb{E}\left[\tilde{B} \mathbb{1}_{\left\{|\tilde{B}| / a_{n} \leq 1\right\}}\right]$, where $\tilde{B}:=$ $B-\mu_{B}$, we can write

$$
\begin{aligned}
\sum_{j=1}^{n} X_{j} \mathbb{1}_{\left\{X_{j} / a_{n} \leq \gamma\right\}} \tilde{B}_{j+1}= & \sum_{j=1}^{n} X_{j} \mathbb{1}_{\left\{X_{j} / a_{n} \leq \gamma\right\}}\left(\tilde{B}_{j+1} \mathbb{1}_{\left\{\left|\tilde{B}_{j+1}\right| / a_{n} \leq 1\right\}}-c_{B, n}\right)+c_{B, n} \sum_{j=1}^{n} X_{j} \mathbb{1}_{\left\{X_{j} / a_{n} \leq \gamma\right\}} \\
& +\sum_{j=1}^{n} X_{j} \mathbb{1}_{\left\{X_{j} / a_{n} \leq \gamma\right\}} \tilde{B}_{j+1} \mathbb{1}_{\left\{\left|\tilde{B}_{j+1}\right| / a_{n}>1\right\}}=: J_{n, \gamma}^{(1)}+J_{n, \gamma}^{(2)}+J_{n, \gamma}^{(3)} .
\end{aligned}
$$

Since

$$
\begin{aligned}
& \mathbb{P}\left(\left|J_{n, \gamma}^{(1)}+J_{n, \gamma}^{(2)}+J_{n, \gamma}^{(3)}\right|>a_{n}^{3 / 2} \epsilon\right) \\
& \leq \mathbb{P}\left(\left|J_{n, \gamma}^{(1)}\right|>a_{n}^{3 / 2} \epsilon / 3\right)+\mathbb{P}\left(\left|J_{n, \gamma}^{(2)}\right|>a_{n}^{3 / 2} \epsilon / 3\right)+\mathbb{P}\left(\left|J_{n, \gamma}^{(3)}\right|>a_{n}^{3 / 2} \epsilon / 3\right), \quad \epsilon>0,
\end{aligned}
$$

to prove (4.5), it is enough to check that $\lim _{\gamma \downarrow 0} \lim _{\sup _{n \rightarrow \infty}} \mathbb{P}\left(\left|J_{n, \gamma}^{(i)}\right|>a_{n}^{3 / 2} \epsilon\right)=0, i=1,2,3$, for all $\epsilon>0$. 
In case of $i=1$, using the independence of $X_{j}$ and $\tilde{B}_{j+1}$, Markov's inequality, and the facts that the summands in $J_{n, \gamma}^{(1)}$ are uncorrelated and $\operatorname{Var}\left[\tilde{B} \mathbb{1}_{\left\{|\tilde{B}| \leq a_{n}\right\}}\right] \leq \mathbb{E}\left[\tilde{B}^{2} \mathbb{1}_{\left\{|\tilde{B}| \leq a_{n}\right\}}\right]$, we have

$$
\begin{aligned}
& \mathbb{P}\left(\left|J_{n, \gamma}^{(1)}\right|>a_{n}^{3 / 2} \epsilon\right) \leq \frac{n}{\epsilon^{2} a_{n}^{3}} \mathbb{E}\left[X_{0}^{2} \mathbb{1}_{\left\{X_{0} / a_{n} \leq \gamma\right\}}\right] \mathbb{E}\left[\tilde{B}^{2} \mathbb{1}_{\left\{|\tilde{B}| \leq a_{n}\right\}}\right] \\
& =\frac{1}{\epsilon^{2}} \frac{\mathbb{E}\left[X_{0}^{2} \mathbb{1}_{\left\{X_{0} / a_{n} \leq \gamma\right\}}\right]}{a_{n}^{2} \gamma^{2} \mathbb{P}\left(X_{0}>a_{n} \gamma\right)} \frac{\mathbb{E}\left[\tilde{B}^{2} \mathbb{1}_{\left\{|\tilde{B}| \leq a_{n}\right\}}\right]}{a_{n}^{2} \mathbb{P}\left(|\tilde{B}|>a_{n}\right)} \gamma^{2} \frac{\mathbb{P}\left(X_{0}>a_{n} \gamma\right)}{\mathbb{P}\left(X_{0}>a_{n}\right)} n \mathbb{P}\left(X_{0}>a_{n}\right) a_{n} \mathbb{P}\left(|\tilde{B}|>a_{n}\right) .
\end{aligned}
$$

Indeed, the summands in $J_{n, \gamma}^{(1)}$ are uncorrelated, since for all $i<j, i, j \in\{1, \ldots, n\}$, we have

$$
\begin{aligned}
& \mathbb{E}\left[X_{i} \mathbb{1}_{\left\{X_{i} / a_{n} \leq \gamma\right\}}\left(\tilde{B}_{i+1} \mathbb{1}_{\left\{\left|\tilde{B}_{i+1}\right| / a_{n} \leq 1\right\}}-c_{B, n}\right) X_{j} \mathbb{1}_{\left\{X_{j} / a_{n} \leq \gamma\right\}}\left(\tilde{B}_{j+1} \mathbb{1}_{\left\{\left|\tilde{B}_{j+1}\right| / a_{n} \leq 1\right\}}-c_{B, n}\right)\right] \\
& =\mathbb{E}\left[X_{i} X_{j} \mathbb{1}_{\left\{X_{i} / a_{n} \leq \gamma\right\}} \mathbb{1}_{\left\{X_{j} / a_{n} \leq \gamma\right\}}\left(\tilde{B}_{i+1} \mathbb{1}_{\left\{\left|\tilde{B}_{i+1}\right| / a_{n} \leq 1\right\}}-c_{B, n}\right)\right] \mathbb{E}\left[\left(\tilde{B}_{j+1} \mathbb{1}_{\left\{\left|\tilde{B}_{j+1}\right| / a_{n} \leq 1\right\}}-c_{B, n}\right)\right] \\
& =\mathbb{E}\left[X_{i} X_{j} \mathbb{1}_{\left\{X_{i} / a_{n} \leq \gamma\right\}} \mathbb{1}_{\left\{X_{j} / a_{n} \leq \gamma\right\}}\left(\tilde{B}_{i+1} \mathbb{1}_{\left\{\left|\tilde{B}_{i+1}\right| / a_{n} \leq 1\right\}}-c_{B, n}\right)\right] \cdot 0=0 .
\end{aligned}
$$

Note that, since $\tilde{B}$ is bounded from below and $B$ is regularly varying with tail index $\alpha$, we have

$$
\mathbb{P}(|\tilde{B}|>x) \sim \mathbb{P}(\tilde{B}>x) \sim \mathbb{P}(B>x) \quad \text { as } x \rightarrow \infty
$$

where we used that for all $\varepsilon>0, \mathbb{P}(B>x(1+\varepsilon)) \leq \mathbb{P}(\tilde{B}>x) \leq \mathbb{P}(B>x)$ for large enough $x$. In particular, $|\tilde{B}|$ is regularly varying with tail index $\alpha$, and moreover, by (1.4),

$$
\mathbb{P}(|\tilde{B}|>x) \sim\left(1-\mu_{A}^{\alpha}\right) \mathbb{P}\left(X_{0}>x\right) \quad \text { as } x \rightarrow \infty .
$$

Consequently, by Karamata's theorem (see, Lemma E.1), Bingham et al. [9, Proposition 1.3.6. (v)], (4.6) and (3.1), for all $\gamma>0$,

$$
\begin{aligned}
\limsup _{n \rightarrow \infty} \mathbb{P}\left(\left|J_{n, \gamma}^{(1)}\right|>a_{n}^{3 / 2} \epsilon\right) & \leq \frac{\alpha^{2} \gamma^{2-\alpha}}{\epsilon^{2}(2-\alpha)^{2}} \limsup _{n \rightarrow \infty}\left(a_{n} \mathbb{P}\left(|\tilde{B}|>a_{n}\right)\right) \\
& =\frac{\alpha^{2} \gamma^{2-\alpha}\left(1-\mu_{A}^{\alpha}\right)}{\epsilon^{2}(2-\alpha)^{2}} \limsup _{n \rightarrow \infty}\left(a_{n} \mathbb{P}\left(X_{0}>a_{n}\right)\right)=\frac{\alpha^{2} \gamma^{2-\alpha}\left(1-\mu_{A}^{\alpha}\right)}{\epsilon^{2}(2-\alpha)^{2}} \limsup _{n \rightarrow \infty} \frac{a_{n}}{n} \\
& =\frac{\alpha^{2} \gamma^{2-\alpha}\left(1-\mu_{A}^{\alpha}\right)}{\epsilon^{2}(2-\alpha)^{2}} \limsup _{n \rightarrow \infty}\left(n^{\frac{1}{\alpha}-1} L(n)\right) \rightarrow 0 \quad \text { as } n \rightarrow \infty
\end{aligned}
$$

In case of $i=2$, since $\mathbb{E}[\tilde{B}]=0$, by Markov's inequality, for all $\epsilon>0$,

$$
\begin{aligned}
\mathbb{P}\left(\left|J_{n, \gamma}^{(2)}\right|>a_{n}^{3 / 2} \epsilon\right) & \leq \frac{n}{\epsilon a_{n}^{3 / 2}} \mathbb{E}\left[X_{0}\right]\left|\mathbb{E}\left[\tilde{B} \mathbb{1}_{\left\{|\tilde{B}| / a_{n} \leq 1\right\}}\right]\right|=\frac{n}{\epsilon a_{n}^{1 / 2}} \mathbb{E}\left[X_{0}\right] \frac{\left|\mathbb{E}\left[\tilde{B} \mathbb{1}_{\left\{|\tilde{B}| / a_{n}>1\right\}}\right]\right|}{a_{n} \mathbb{P}\left(|\tilde{B}|>a_{n}\right)} \mathbb{P}\left(|\tilde{B}|>a_{n}\right) \\
& \leq \frac{\mathbb{E}\left[X_{0}\right]}{\epsilon a_{n}^{1 / 2}} \frac{\mathbb{E}\left[|\tilde{B}| \mathbb{1}_{\left\{|\tilde{B}|>a_{n}\right\}}\right]}{a_{n} \mathbb{P}\left(|\tilde{B}|>a_{n}\right)} n \mathbb{P}\left(|\tilde{B}|>a_{n}\right) \rightarrow 0 \quad \text { as } n \rightarrow \infty,
\end{aligned}
$$


since, by Karamata's theorem (see, Lemma E.1),

$$
\lim _{n \rightarrow \infty} \frac{\mathbb{E}\left[|\tilde{B}| \mathbb{1}_{\left\{|\tilde{B}|>a_{n}\right\}}\right]}{a_{n} \mathbb{P}\left(|\tilde{B}|>a_{n}\right)}=\frac{\alpha}{\alpha-1}
$$

and, by (4.6) and (3.1), $n \mathbb{P}\left(|\tilde{B}|>a_{n}\right) \rightarrow 1-\mu_{A}^{\alpha} \in(0,1)$ as $n \rightarrow \infty$.

In case of $i=3$, similarly as in case of $i=2$, using Markov's inequality and the independence of $X_{0}$ and $B_{1}$, for all $\epsilon, \gamma>0$, we have

$$
\begin{aligned}
\mathbb{P}\left(\left|J_{n, \gamma}^{(3)}\right|>a_{n}^{3 / 2} \epsilon\right) & \leq \frac{n \mathbb{E}\left(X_{0} \mathbb{1}_{\left\{X_{0} / a_{n} \leq \gamma\right\}}\left|\tilde{B}_{1}\right| \mathbb{1}_{\left\{\left|\tilde{B}_{1}\right|>a_{n}\right\}}\right)}{a_{n}^{3 / 2} \epsilon} \\
& \leq \frac{n}{\epsilon a_{n}^{1 / 2}} \mathbb{E}\left[X_{0}\right] \frac{\mathbb{E}\left[|\tilde{B}| \mathbb{1}_{\left\{|\tilde{B}|>a_{n}\right\}}\right]}{a_{n} \mathbb{P}\left(|\tilde{B}|>a_{n}\right)} \mathbb{P}\left(|\tilde{B}|>a_{n}\right) \rightarrow 0 \quad \text { as } n \rightarrow \infty,
\end{aligned}
$$

hence we conclude (4.1).

Step 4. Finally, we determine the characteristic function of the random vector $\left(V^{(1)}, V^{(2)}\right)$. Using the continuity theorem, conditioning on $\left(P_{i}\right)_{i \in \mathbb{N}}$ and applying the portmanteau lemma, we have for $s, t \in \mathbb{R}$

$$
\begin{aligned}
\mathbb{E}\left[\mathrm{e}^{\mathrm{i}\left(s V^{(1)}+t V^{(2)}\right)}\right] & =\mathbb{E}\left[\exp \left\{\mathrm{i}\left(\frac{s}{1-\mu_{A}^{2}} \sum_{i=1}^{\infty} P_{i}^{2}+\frac{t}{\left(1-\mu_{A}^{3}\right)^{1 / 2}} \sum_{i=1}^{\infty} P_{i}^{3 / 2} Z_{i}\right)\right\}\right] \\
& =\lim _{n \rightarrow \infty} \mathbb{E}\left[\exp \left\{\mathrm{i}\left(\frac{s}{1-\mu_{A}^{2}} \sum_{i=1}^{n} P_{i}^{2}+\frac{t}{\left(1-\mu_{A}^{3}\right)^{1 / 2}} \sum_{i=1}^{n} P_{i}^{3 / 2} Z_{i}\right)\right\}\right] \\
& =\lim _{n \rightarrow \infty} \mathbb{E}\left[\mathbb{E}\left[\exp \left\{\mathrm{i}\left(\frac{s}{1-\mu_{A}^{2}} \sum_{i=1}^{n} P_{i}^{2}+\frac{t}{\left(1-\mu_{A}^{3}\right)^{1 / 2}} \sum_{i=1}^{n} P_{i}^{3 / 2} Z_{i}\right)\right\} \mid\left(P_{i}\right)_{i \in \mathbb{N}}\right]\right] \\
& =\lim _{n \rightarrow \infty} \mathbb{E}\left[\exp \left\{\frac{\mathrm{i} s}{1-\mu_{A}^{2}} \sum_{i=1}^{n} P_{i}^{2}-\frac{\sigma_{A}^{2} t^{2}}{2\left(1-\mu_{A}^{3}\right)} \sum_{i=1}^{n} P_{i}^{3}\right\}\right] \\
& =\mathbb{E}\left[\exp \left\{\frac{\mathrm{i} s}{1-\mu_{A}^{2}} \sum_{i=1}^{\infty} P_{i}^{2}-\frac{\sigma_{A}^{2} t^{2}}{2\left(1-\mu_{A}^{3}\right)} \sum_{i=1}^{\infty} P_{i}^{3}\right\}\right]
\end{aligned}
$$

since the series $\sum_{i=1}^{\infty} P_{i}^{\beta}$ and $\sum_{i=1}^{\infty} P_{i}^{3 / 2} Z_{i}$ are convergent almost surely for any $\beta \in(\alpha, \infty)$ (see Step 2). As in the proof of Campbell's theorem (see, e.g., Kingman [23, Section 3.2]), one can prove that

$$
\mathbb{E}\left[\exp \left\{u \sum_{i=1}^{\infty} P_{i}^{2}+v \sum_{i=1}^{\infty} P_{i}^{3}\right\}\right]=\exp \left\{\int_{0}^{\infty}\left(\mathrm{e}^{u y^{2}+v y^{3}}-1\right) \theta \alpha y^{-\alpha-1} \mathrm{~d} y\right\}
$$

for any $u, v \in \mathbb{C}$ with $\Re(u) \leq 0$ and $\Re(v) \leq 0$, where $\Re(z)$ denotes the real part of $z \in \mathbb{C}$. Indeed, (4.7) holds for $u, v \in(-\infty, 0]$ by Campbell's theorem with the function $(0, \infty) \ni y \mapsto u y^{2}+v y^{3}$ satisfying $\int_{0}^{\infty}\left(\left|u y^{2}+v y^{3}\right| \wedge 1\right) \theta \alpha y^{-\alpha-1} \mathrm{~d} y<\infty$. Since for any given $u \in \mathbb{C}$ with $\Re(u) \leq 0$, 
both sides of (4.7) as functions of $v$ are analytic functions on $\{v \in \mathbb{C}: \Re(v)<0\}$, and for any given $v \in \mathbb{C}$ with $\Re(v) \leq 0$, both sides of (4.7) as functions of $u$ are analytic functions on $\{u \in \mathbb{C}: \Re(u)<0\}$, Hartogs's theorem yields that both sides of (4.7) are analytic functions on $\left\{(u, v) \in \mathbb{C}^{2}: \Re(u)<0, \Re(v)<0\right\}$. So, by the identity theorem for analytic functions, (4.7) holds on $\left\{(u, v) \in \mathbb{C}^{2}: \Re(u)<0, \Re(v)<0\right\}$. Both sides of $(4.7)$ are continuous functions on $\left\{(u, v) \in \mathbb{C}^{2}: \Re(u) \leq 0, \Re(v) \leq 0\right\}$, so $(4.7)$ holds on $\left\{(u, v) \in \mathbb{C}^{2}: \Re(u) \leq 0, \Re(v) \leq 0\right\}$. Applying (4.7) for $u=\mathrm{i} s /\left(1-\mu_{A}^{2}\right)$ and $v=-\sigma_{A}^{2} t^{2} /\left(2\left(1-\mu_{A}^{3}\right)\right)$, we obtain the formula for $\mathbb{E}\left[\mathrm{e}^{\mathrm{i}\left(s V^{(1)}+t V^{(2)}\right)}\right], s, t, \in \mathbb{R}$.

For each $\beta \in(0,1)$ and $z \in \mathbb{R}$, we have

$$
\int_{0}^{\infty}\left(\mathrm{e}^{\mathrm{i} z r}-1\right) r^{-1-\beta} \mathrm{d} r=\Gamma(-\beta) \cos \left(\frac{\pi \beta}{2}\right)|z|^{\beta}\left(1-\mathrm{i} \tan \left(\frac{\pi \beta}{2}\right) \operatorname{sgn}(z)\right),
$$

see, e.g., the proof of Theorem 14.10 in Sato [38]. Applying (4.8) for $\beta=\alpha / 2$ and $z=s /\left(1-\mu_{A}^{2}\right)$, we obtain for $s \in \mathbb{R}$

$$
\begin{aligned}
\mathbb{E}\left[\mathrm{e}^{\mathrm{i} s V^{(1)}}\right] & =\exp \left\{\theta \int_{0}^{\infty}\left(\exp \left\{\frac{\mathrm{i} s}{1-\mu_{A}^{2}} y^{2}\right\}-1\right) \alpha y^{-\alpha-1} \mathrm{~d} y\right\} \\
& =\exp \left\{\theta \int_{0}^{\infty}\left(\exp \left\{\frac{\mathrm{i} s}{1-\mu_{A}^{2}} r\right\}-1\right) \frac{\alpha}{2} r^{-\frac{\alpha}{2}-1} \mathrm{~d} r\right\} \\
& =\exp \left\{\theta \frac{\alpha}{2} \Gamma\left(-\frac{\alpha}{2}\right) \cos \left(\frac{\pi \alpha}{4}\right)\left|\frac{s}{1-\mu_{A}^{2}}\right|^{\alpha / 2}\left(1-\mathrm{i} \tan \left(\frac{\pi \alpha}{4}\right) \operatorname{sgn}\left(\frac{s}{1-\mu_{A}^{2}}\right)\right)\right\} \\
& =\exp \left\{-\frac{\theta}{\left(1-\mu_{A}^{2}\right)^{\alpha / 2}} \Gamma\left(1-\frac{\alpha}{2}\right) \cos \left(\frac{\pi \alpha}{4}\right)|s|^{\alpha / 2}\left(1-\mathrm{itan}\left(\frac{\pi \alpha}{4}\right) \operatorname{sgn}(s)\right)\right\}
\end{aligned}
$$

hence we obtain the characteristic function of $V^{(1)}$.

For each $\beta \in(0,1)$ and $z \in[0, \infty)$, we have

$$
\int_{0}^{\infty}\left(1-\mathrm{e}^{-z r}\right) \beta r^{-1-\beta} \mathrm{d} r=\Gamma(1-\beta) z^{\beta}
$$

see, e.g., Example 1.4 in Li [28] or the method of the proof of Example 8.11 in Sato [38]. Applying (4.9) for $\beta=\alpha / 3$ and $z=\sigma_{A}^{2} t^{2} /\left(2\left(1-\mu_{A}^{3}\right)\right)$, we obtain for $t \in \mathbb{R}$

$$
\begin{aligned}
\mathbb{E}\left[\mathrm{e}^{\mathrm{i} t V^{(2)}}\right] & =\exp \left\{\theta \int_{0}^{\infty}\left(\exp \left\{-\frac{\sigma_{A}^{2} t^{2}}{2\left(1-\mu_{A}^{3}\right)} y^{3}\right\}-1\right) \alpha y^{-\alpha-1} \mathrm{~d} y\right\} \\
& =\exp \left\{\theta \int_{0}^{\infty}\left(\exp \left\{-\frac{\sigma_{A}^{2} t^{2}}{2\left(1-\mu_{A}^{3}\right)} r\right\}-1\right) \frac{\alpha}{3} r^{-\frac{\alpha}{3}-1} \mathrm{~d} r\right\} \\
& =\exp \left\{-\theta \Gamma\left(1-\frac{\alpha}{3}\right)\left(\frac{\sigma_{A}^{2} t^{2}}{2\left(1-\mu_{A}^{3}\right)}\right)^{\alpha / 3}\right\},
\end{aligned}
$$

hence we obtain the characteristic function of $V^{(2)}$, and this finishes the proof. 
Remark 4.2. Note that the law of $\left(V^{(1)}, V^{(2)}\right)$ in $(4.1)$ does not depend on the choice of the scaling sequence of $\left(a_{n}\right)_{n \in \mathbb{N}}$ satisfying (3.1), which can be seen from the form of the characteristic function of $\left(V^{(1)}, V^{(2)}\right)$ given in Theorem 4.1.

Remark 4.3. One can check that if $\alpha \in(1,3 / 2)$, then the series in the definition of $V^{(2)}$ in Theorem 4.1 is absolutely convergent almost surely. By the mapping and marking theorems (see, e.g., Kingman [23, Sections 2.3 and 5.2]), we have $\sum_{i=1}^{\infty} \delta_{\left(P_{i}^{3 / 2},\left|Z_{i}\right|\right)}$ is a Poisson random measure on $(0, \infty) \times(0, \infty)$ with intensity measure $\theta \mathrm{d}\left(-y^{-2 \alpha / 3}\right) \times f_{\left|Z_{1}\right|}(z) \mathrm{d} z$, where $f_{\left|Z_{1}\right|}$ denotes the density function of $\left|Z_{1}\right|$. Using again the mapping theorem, $\sum_{i=1}^{\infty} \delta_{P_{i}^{3 / 2}\left|Z_{i}\right|}$ is a Poisson random measure on $(0, \infty)$ with intensity measure $\theta \mathbb{E}\left[\left|Z_{1}\right|^{2 \alpha / 3}\right] \mathrm{d}\left(-y^{-2 \alpha / 3}\right)$, since for any $t>0$,

$$
\begin{aligned}
& \int_{\left\{(y, z) \in(0, \infty)^{2}: y z>t\right\}} \theta f_{\left|Z_{1}\right|}(z) \mathrm{d}\left(-y^{-2 \alpha / 3}\right) \mathrm{d} z=\int_{t}^{\infty} \int_{0}^{\infty} \frac{2 \alpha \theta}{3}\left(\frac{u}{z}\right)^{-\frac{2 \alpha}{3}-1} f_{\left|Z_{1}\right|}(z) \frac{1}{z} \mathrm{~d} u \mathrm{~d} z \\
& =\frac{2 \alpha \theta}{3} \mathbb{E}\left[\left|Z_{1}\right|^{2 \alpha / 3}\right] \int_{t}^{\infty} u^{-\frac{2 \alpha}{3}-1} \mathrm{~d} u=\theta \mathbb{E}\left[\left|Z_{1}\right|^{2 \alpha / 3}\right] \int_{t}^{\infty} 1 \mathrm{~d}\left(-u^{-2 \alpha / 3}\right) .
\end{aligned}
$$

Hence, by Campbell's theorem, we have $\sum_{i=1}^{\infty} P_{i}^{3 / 2}\left|Z_{i}\right|$ is convergent almost surely, since now $0<2 \alpha / 3<1$ and then

$$
\int_{0}^{\infty}(y \wedge 1) \theta \mathbb{E}\left[\left|Z_{1}\right|^{2 \alpha / 3}\right] \mathrm{d}\left(-y^{-2 \alpha / 3}\right)=\frac{2 \alpha \theta}{3} \mathbb{E}\left[\left|Z_{1}\right|^{2 \alpha / 3}\right]\left(\int_{0}^{1} y^{-2 \alpha / 3} \mathrm{~d} y+\int_{1}^{\infty} y^{-2 \alpha / 3-1} \mathrm{~d} y\right)<\infty .
$$

Further, since $\int_{0}^{\infty} y \theta \mathbb{E}\left[\left|Z_{1}\right|^{2 \alpha / 3}\right] \mathrm{d}\left(-y^{-2 \alpha / 3}\right)=\infty$, by Kingman [23, formula (3.18)], we have $\mathbb{E}\left[\sum_{i=1}^{\infty} P_{i}^{3 / 2}\left|Z_{i}\right|\right]=\infty$.

\section{On the limit behavior of the CLS estimator}

Now we can formulate our main result.

Theorem 5.1. We have

$$
\sqrt{a_{n}}\left({\widehat{\mu_{A}}}^{(n)}-\mu_{A}\right) \stackrel{d}{\longrightarrow} \frac{V^{(2)}}{V^{(1)}} \quad \text { as } n \rightarrow \infty,
$$

where the sequence $\left(a_{n}\right)_{n \in \mathbb{N}}$ and the joint characteristic function of $\left(V^{(1)}, V^{(2)}\right)$ is given in (3.1) and in Theorem 4.1, respectively.

Proof. By Theorem 4.1, $V^{(1)}$ is an $\alpha / 2$-stable positive random variable, thus it is absolutely continuous and $\mathbb{P}\left(V^{(1)}>0\right)=\mathbb{P}\left(V^{(1)} \neq 0\right)=1$. For each $n \in \mathbb{N}$, by the strong stationarity of

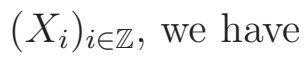

$$
\sqrt{a_{n}}\left(\widehat{\mu}_{A}^{(n)}-\mu_{A}\right) \stackrel{d}{=} \frac{\sum_{j=1}^{n} X_{j} M_{j+1} / a_{n}^{3 / 2}}{\sum_{j=1}^{n}\left(X_{j} / a_{n}\right)^{2}} .
$$

Consequently, by Theorem 4.1 and the continuous mapping theorem (see, e.g., Billingsley [8, Theorem 5.1]), we conclude the statement. 
Remark 5.2. The limit law $V^{(2)} / V^{(1)}$ in Theorem 5.1 can be written in the form

$$
\frac{V^{(2)}}{V^{(1)}} \stackrel{d}{=} \frac{\left(1-\mu_{A}^{2}\right) \sigma_{A}}{\left(1-\mu_{A}^{3}\right)^{1 / 2}\left(1-\mu_{A}^{\alpha}\right)^{\frac{1}{2 \alpha}}} \frac{\sum_{i=1}^{\infty}\left(\widetilde{P}_{i}\right)^{\frac{3}{2}} \widetilde{Z}_{i}}{\sum_{i=1}^{\infty}\left(\widetilde{P}_{i}\right)^{2}}
$$

where $\sum_{i=1}^{\infty} \delta_{\widetilde{P}_{i}}$ is a Poisson random measure on $(0, \infty)$ with intensity measure $\mathrm{d}\left(-y^{-\alpha}\right)$ being independent of an i.i.d. sequence of $\mathcal{N}(0,1)$-distributed random variables $\left(\widetilde{Z}_{i}\right)_{i \in \mathbb{N}}$. Indeed, using the mapping theorem for Poisson random measures, one can check that $\sum_{i=1}^{\infty} \delta_{\theta^{-\frac{1}{\alpha}} P_{i}}$ is a Poisson random measure on $(0, \infty)$ with intensity measure $\mathrm{d}\left(-y^{-\alpha}\right)$. Consequently, we have

$$
\begin{aligned}
\left(V^{(1)}, V^{(2)}\right) & \stackrel{d}{=}\left(\frac{1}{1-\mu_{A}^{2}} \theta^{\frac{2}{\alpha}} \sum_{i=1}^{\infty}\left(\theta^{-\frac{1}{\alpha}} P_{i}\right)^{2}, \frac{1}{\left(1-\mu_{A}^{3}\right)^{1 / 2}} \theta^{\frac{3}{2 \alpha}} \sum_{i=1}^{\infty}\left(\theta^{-\frac{1}{\alpha}} P_{i}\right)^{\frac{3}{2}} Z_{i}\right) \\
& \stackrel{d}{=}\left(\frac{\left(1-\mu_{A}^{\alpha}\right)^{\frac{2}{\alpha}}}{1-\mu_{A}^{2}} \sum_{i=1}^{\infty}\left(\widetilde{P}_{i}\right)^{2}, \frac{\left(1-\mu_{A}^{\alpha}\right)^{\frac{3}{2 \alpha}} \sigma_{A}}{\left(1-\mu_{A}^{3}\right)^{1 / 2}} \sum_{i=1}^{\infty}\left(\widetilde{P}_{i}\right)^{\frac{3}{2}} \widetilde{Z}_{i}\right),
\end{aligned}
$$

yielding the statement.

Note that $\sum_{i=1}^{\infty}\left(\widetilde{P}_{i}\right)^{\frac{3}{2}} \widetilde{Z}_{i} / \sum_{i=1}^{\infty}\left(\widetilde{P}_{i}\right)^{2}$ does not depend on the parameter $\mu_{A}$ to be estimated nor on $\sigma_{A}$. This gives the possibility for formulating a version of Theorem 5.1 with a random normalization such that the limit law does not depend on $\mu_{A}$ and $\sigma_{A}$.

In what follows, we collect several interesting properties of $\left(V^{(1)}, V^{(2)}\right)$ and $V^{(2)} / V^{(1)}$. The characteristic function of a random vector $\boldsymbol{X}$ will be denoted by $\varphi_{\boldsymbol{X}}$.

Proposition 5.3. The distribution of $\left(V^{(1)}, V^{(2)}\right)$ is operator stable, and the matrix $\operatorname{diag}_{2}\left(\frac{2}{\alpha}, \frac{3}{2 \alpha}\right)$ is an exponent of it. Particularly, the distribution of $\left(V^{(1)}, V^{(2)}\right)$ is full and infinitely divisible, and has an infinitely differentiable density function, and the partial derivatives of this density function tend to 0 at infinity.

Proof. First, observe that for each $a \in(0, \infty)$ and $s, t \in \mathbb{R}$, by the substitution $a^{1 / \alpha} y=x$, we obtain

$$
\begin{aligned}
& \varphi_{a^{2 / \alpha} V^{(1)}, a^{3 /(2 \alpha)} V^{(2)}}(s, t) \\
& =\exp \left\{\theta \int_{0}^{\infty}\left(\exp \left\{\frac{\mathrm{i} a^{2 / \alpha} s}{1-\mu_{A}^{2}} y^{2}-\frac{\sigma_{A}^{2}\left(a^{3 /(2 \alpha)} t\right)^{2}}{2\left(1-\mu_{A}^{3}\right)} y^{3}\right\}-1\right) \alpha y^{-\alpha-1} \mathrm{~d} y\right\} \\
& =\exp \left\{a \theta \int_{0}^{\infty}\left(\exp \left\{\frac{\mathrm{i} s}{1-\mu_{A}^{2}} x^{2}-\frac{\sigma_{A}^{2} t^{2}}{2\left(1-\mu_{A}^{3}\right)} x^{3}\right\}-1\right) \alpha x^{-\alpha-1} \mathrm{~d} x\right\} \\
& =\left(\varphi_{V^{(1)}, V^{(2)}}(s, t)\right)^{a}
\end{aligned}
$$

hence Equation (7.8) in Meerschaert and Scheffler [33] is satisfied with exponent $\operatorname{diag}_{2}\left(\frac{2}{\alpha}, \frac{3}{2 \alpha}\right)$ and without shifts. 
Particularly, with $a=n^{-1}, n \in \mathbb{N}$, we get

$$
\varphi_{V^{(1)}, V^{(2)}}(s, t)=\left(\varphi_{n^{-2 / \alpha} V^{(1)}, n^{-3 /(2 \alpha)} V^{(2)}}(s, t)\right)^{n}
$$

for all $s, t \in \mathbb{R}$ and $n \in \mathbb{N}$, hence the distribution of $\left(V^{(1)}, V^{(2)}\right)$ is infinitely divisible.

In order to prove that the distribution of $\left(V^{(1)}, V^{(2)}\right)$ is full, we have to show that for each $\left(v_{1}, v_{2}\right) \in \mathbb{R}^{2} \backslash\{(0,0)\}$, the random variable $v_{1} V^{(1)}+v_{2} V^{(2)}$ is nondegenerate. If we suppose that, on the contrary, there exist $\left(v_{1}, v_{2}\right) \in \mathbb{R}^{2} \backslash\{(0,0)\}$ and $x_{0} \in \mathbb{R}$ such that $v_{1} V^{(1)}+v_{2} V^{(2)}=x_{0}$ almost surely, then for each $t \in \mathbb{R}$, we would have

$$
\begin{aligned}
\mathrm{e}^{\mathrm{i} t x_{0}} & =\varphi_{v_{1} V^{(1)}+v_{2} V^{(2)}}(t)=\varphi_{V^{(1)}, V^{(2)}}\left(v_{1} t, v_{2} t\right) \\
& =\exp \left\{\theta \int_{0}^{\infty}\left(\exp \left\{\frac{\mathrm{i} v_{1} t}{1-\mu_{A}^{2}} y^{2}-\frac{\sigma_{A}^{2}\left(v_{2} t\right)^{2}}{2\left(1-\mu_{A}^{3}\right)} y^{3}\right\}-1\right) \alpha y^{-\alpha-1} \mathrm{~d} y\right\} .
\end{aligned}
$$

We have $\varphi_{V^{(1)}, V^{(2)}}\left(v_{1} t, v_{2} t\right) \neq 0$ for any $t \in \mathbb{R}$, since the distribution of $\left(V^{(1)}, V^{(2)}\right)$ is infinitely divisible. Applying Lemma 7.6 in Sato [38], we would obtain

$$
\mathrm{i} t x_{0}=\theta \int_{0}^{\infty}\left(\exp \left\{\frac{\mathrm{i} v_{1} t}{1-\mu_{A}^{2}} y^{2}-\frac{\sigma_{A}^{2} v_{2}^{2} t^{2}}{2\left(1-\mu_{A}^{3}\right)} y^{3}\right\}-1\right) \alpha y^{-\alpha-1} \mathrm{~d} y, \quad t \in \mathbb{R} .
$$

Taking the real parts of both sides, we would get

$$
0=\theta \int_{0}^{\infty}\left(\exp \left\{-\frac{\sigma_{A}^{2} v_{2}^{2} t^{2}}{2\left(1-\mu_{A}^{3}\right)} y^{3}\right\} \cos \left(\frac{v_{1} t}{1-\mu_{A}^{2}} y^{2}\right)-1\right) \alpha y^{-\alpha-1} \mathrm{~d} y, \quad t \in \mathbb{R} .
$$

Since the integrand is continuous and nonpositive, we would conclude that it is identically zero, yielding that

$$
\cos \left(\frac{v_{1} t}{1-\mu_{A}^{2}} y^{2}\right)=\exp \left\{\frac{\sigma_{A}^{2} v_{2}^{2} t^{2}}{2\left(1-\mu_{A}^{3}\right)} y^{3}\right\}, \quad y \in[0, \infty), \quad t \in \mathbb{R},
$$

which is a contradiction due to $\left(v_{1}, v_{2}\right) \neq(0,0)$, hence the distribution of $\left(V^{(1)}, V^{(2)}\right)$ is full. By Theorem 7.2.1 in Meerschaert and Scheffler [33], taking into account (5.1), we obtain that the distribution of $\left(V^{(1)}, V^{(2)}\right)$ is operator stable and the matrix $\operatorname{diag}_{2}\left(\frac{2}{\alpha}, \frac{3}{2 \alpha}\right)$ is an exponent of it. For the facts that $\left(V^{(1)}, V^{(2)}\right)$ has an infinitely differentiable density function, and the partial derivatives of this density function tend to 0 at infinity, see Luczak [32, Corollary 2.1] and Kern and Wedrich [22, page 387].

Proposition 5.4. The random variables $V^{(1)}$ and $V^{(2)}$ are dependent.

Proof. If we suppose that, on the contrary, $V^{(1)}$ and $V^{(2)}$ are independent, then we would have $\varphi_{V^{(1)}, V^{(2)}}(s, t)=\varphi_{V^{(1)}}(s) \varphi_{V^{(2)}}(t)$ for all $s, t \in \mathbb{R}$, hence

$$
\exp \left\{\theta \int_{0}^{\infty}\left(\exp \left\{\frac{\mathrm{i} s}{1-\mu_{A}^{2}} y^{2}-\frac{\sigma_{A}^{2} t^{2}}{2\left(1-\mu_{A}^{3}\right)} y^{3}\right\}-1\right) \alpha y^{-\alpha-1} \mathrm{~d} y\right\}
$$




$$
\begin{aligned}
= & \exp \left\{\theta \int_{0}^{\infty}\left(\exp \left\{\frac{\mathrm{i} s}{1-\mu_{A}^{2}} y^{2}\right\}-1\right) \alpha y^{-\alpha-1} \mathrm{~d} y\right\} \\
& \times \exp \left\{\theta \int_{0}^{\infty}\left(\exp \left\{-\frac{\sigma_{A}^{2} t^{2}}{2\left(1-\mu_{A}^{3}\right)} y^{3}\right\}-1\right) \alpha y^{-\alpha-1} \mathrm{~d} y\right\} \\
= & \exp \left\{\theta \int_{0}^{\infty}\left(\exp \left\{\frac{\mathrm{i} s}{1-\mu_{A}^{2}} y^{2}\right\}+\exp \left\{-\frac{\sigma_{A}^{2} t^{2}}{2\left(1-\mu_{A}^{3}\right)} y^{3}\right\}-2\right) \alpha y^{-\alpha-1} \mathrm{~d} y\right\}
\end{aligned}
$$

for all $s, t \in \mathbb{R}$. We have $\varphi_{V^{(1)}, V^{(2)}}(s, t) \neq 0, \varphi_{V^{(1)}}(s) \neq 0$ and $\varphi_{V^{(2)}}(t) \neq 0$ for any $s, t \in \mathbb{R}$, since the distributions of $\left(V^{(1)}, V^{(2)}\right), V^{(1)}$ and $V^{(2)}$ are infinitely divisible. Applying Lemma 7.6 in Sato [38], we would obtain

$$
\begin{aligned}
& \theta \int_{0}^{\infty}\left(\exp \left\{\frac{\mathrm{i} s}{1-\mu_{A}^{2}} y^{2}-\frac{\sigma_{A}^{2} t^{2}}{2\left(1-\mu_{A}^{3}\right)} y^{3}\right\}-1\right) \alpha y^{-\alpha-1} \mathrm{~d} y \\
& =\theta \int_{0}^{\infty}\left(\exp \left\{\frac{\mathrm{i} s}{1-\mu_{A}^{2}} y^{2}\right\}+\exp \left\{-\frac{\sigma_{A}^{2} t^{2}}{2\left(1-\mu_{A}^{3}\right)} y^{3}\right\}-2\right) \alpha y^{-\alpha-1} \mathrm{~d} y
\end{aligned}
$$

for all $s, t \in \mathbb{R}$, hence

$$
\int_{0}^{\infty}\left(\exp \left\{\frac{\mathrm{i} s}{1-\mu_{A}^{2}} y^{2}\right\}-1\right)\left(\exp \left\{-\frac{\sigma_{A}^{2} t^{2}}{2\left(1-\mu_{A}^{3}\right)} y^{3}\right\}-1\right) \alpha y^{-\alpha-1} \mathrm{~d} y=0
$$

for all $s, t \in \mathbb{R}$. Taking the real parts of both sides, we would get

$$
\int_{0}^{\infty}\left(\cos \left(\frac{s}{1-\mu_{A}^{2}} y^{2}\right)-1\right)\left(\exp \left\{-\frac{\sigma_{A}^{2} t^{2}}{2\left(1-\mu_{A}^{3}\right)} y^{3}\right\}-1\right) \alpha y^{-\alpha-1} \mathrm{~d} y=0
$$

for all $s, t \in \mathbb{R}$. Since the integrand is continuous and nonnegative, we would conclude that it is identically zero, yielding a contradiction unless $s=t=0$, hence the random variables $V^{(1)}$ and $V^{(2)}$ are dependent.

Note that, by Theorem 4.1, $\mathbb{E}\left[V^{(1)}\right]=\infty$ for all $\alpha \in(1,2)$, and $\mathbb{E}\left[V^{(2)}\right]$ does not exist if $\alpha \in\left(1, \frac{3}{2}\right]$ and $\mathbb{E}\left[V^{(2)}\right]=0$ if $\alpha \in\left(\frac{3}{2}, 2\right)$. In what follows we show that all the exponential moments of $V^{(2)} / V^{(1)}$ are finite.

Proposition 5.5. For each $t \in \mathbb{R}$, we have

$$
\mathbb{E}\left[\exp \left\{t \frac{V^{(2)}}{V^{(1)}}\right\}\right]<\infty .
$$

Proof. Let $t \in \mathbb{R}$ be fixed. Using that the series $\sum_{i=1}^{\infty} P_{i}^{\beta}$ and $\sum_{i=1}^{\infty} P_{i}^{3 / 2} Z_{i}$ are convergent almost surely for any $\beta \in(\alpha, \infty)$ (see Step 2 of the proof of Theorem 4.1), conditioning on $\left(P_{i}\right)_{i \in \mathbb{N}}$, by the continuity theorem, we have

$$
\mathbb{E}\left[\exp \left\{t \frac{V^{(2)}}{V^{(1)}}\right\}\right]=\mathbb{E}\left[\exp \left\{\frac{t^{2} \sigma_{A}^{2}\left(1-\mu_{A}^{2}\right)^{2}}{2\left(1-\mu_{A}^{3}\right)} \frac{\sum_{i=1}^{\infty} P_{i}^{3}}{\left(\sum_{i=1}^{\infty} P_{i}^{2}\right)^{2}}\right\}\right] .
$$


So we need to check that all the exponential moments of

$$
U:=\theta^{1 / \alpha} \frac{\sum_{i=1}^{\infty} P_{i}^{3}}{\left(\sum_{i=1}^{\infty} P_{i}^{2}\right)^{2}}
$$

are finite. Then $\mathbb{P}(U \in(0, \infty))=1$, since the series $\sum_{i=1}^{\infty} P_{i}^{2}$ and $\sum_{i=1}^{\infty} P_{i}^{3}$ are absolutely convergent with positive sums almost surely. Recall that the Poisson point process $\left(P_{i}\right)_{i \in \mathbb{N}}$ in Theorem 4.1 can be represented as

$$
\left(P_{i}\right)_{i \in \mathbb{N}} \stackrel{d}{=}\left(\theta^{1 / \alpha} \Gamma_{i}^{-1 / \alpha}\right)_{i \in \mathbb{N}}
$$

with $\Gamma_{i}=E_{1}+\ldots+E_{i}, i \in \mathbb{N}$, where $E_{j}, j \in \mathbb{N}$, are i.i.d. random variables with an exponential distribution with parameter 1 independent of $\left(Z_{i}\right)_{i \in \mathbb{N}}$. Hence

$$
U \stackrel{d}{=} \frac{\sum_{i=1}^{\infty} \Gamma_{i}^{-3 / \alpha}}{\left(\sum_{i=1}^{\infty} \Gamma_{i}^{-2 / \alpha}\right)^{2}}
$$

Since

$$
\left(\sum_{i=1}^{\infty} \Gamma_{i}^{-2 / \alpha}\right)^{2} \geq \sum_{i=1}^{\infty} \Gamma_{i}^{-4 / \alpha}+\Gamma_{1}^{-2 / \alpha} \sum_{i=2}^{\infty} \Gamma_{i}^{-2 / \alpha}
$$

we see that $U>x$ with $x>0$, implies that

$$
\Gamma_{1}^{-3 / \alpha}>\Gamma_{1}^{-4 / \alpha} x \quad \text { or } \quad \Gamma_{i}^{-3 / \alpha}>\left(\Gamma_{i}^{-4 / \alpha}+\Gamma_{1}^{-2 / \alpha} \Gamma_{i}^{-2 / \alpha}\right) x \quad \text { for some } i \geq 2 .
$$

In both cases we have $\Gamma_{1}>x^{\alpha}$. Indeed, if $\Gamma_{i}^{-3 / \alpha}>\left(\Gamma_{i}^{-4 / \alpha}+\Gamma_{1}^{-2 / \alpha} \Gamma_{i}^{-2 / \alpha}\right) x$ with some $i \geq 2$, then $\Gamma_{i}^{-3 / \alpha}>\Gamma_{1}^{-2 / \alpha} \Gamma_{i}^{-2 / \alpha} x$ yielding that $\Gamma_{1}^{2 / \alpha} \Gamma_{i}^{-1 / \alpha}>x$, and then, since $\Gamma_{1} \leq \Gamma_{i}$, we have $x<\Gamma_{1}^{1 / \alpha}$, as desired. Summarizing, we have shown that

$$
\mathbb{P}(U>x) \leq \mathbb{P}\left(\Gamma_{1}>x^{\alpha}\right)=\mathrm{e}^{-x^{\alpha}}, \quad x>0,
$$

which yields the statement. Indeed, if $s \leq 0$, then $\mathbb{E}\left[\mathrm{e}^{s U}\right] \leq 1<\infty$, since $\mathbb{P}(U \in(0, \infty))=1$, and if $s>0$, then

$$
\begin{aligned}
\mathbb{E}\left[\mathrm{e}^{s U}\right] & =\int_{0}^{\infty} \mathbb{P}\left(\mathrm{e}^{s U}>x\right) \mathrm{d} x=\int_{0}^{\infty} \mathbb{P}(U>\ln (x) / s) \mathrm{d} x \leq \int_{0}^{\mathrm{e}^{s}} 1 \mathrm{~d} x+\int_{\mathrm{e}^{s}}^{\infty} \mathrm{e}^{-(\ln (x) / s)^{\alpha}} \mathrm{d} x \\
& =\mathrm{e}^{s}+\int_{s}^{\infty} \mathrm{e}^{y-(y / s)^{\alpha}} \mathrm{d} y<\infty,
\end{aligned}
$$

since $\alpha \in(1,2)$, and $y-(y / s)^{\alpha}<-1 /\left(2 s^{\alpha}\right) y$ for large enough $y>s$.

Proposition 5.6. The random variable $V^{(2)} / V^{(1)}$ has a continuously differentiable density function.

Proof. Using that the series $\sum_{i=1}^{\infty} P_{i}^{\beta}$ and $\sum_{i=1}^{\infty} P_{i}^{3 / 2} Z_{i}$ are convergent almost surely for any $\beta \in(\alpha, \infty)$ (see Step 2 of the proof of Theorem 4.1), conditioning on $\left(P_{i}\right)_{i \in \mathbb{N}}$, by the continuity theorem, for any $t \in \mathbb{R}$, we have

$$
\mathbb{E}\left[\exp \left\{\mathrm{i} t \frac{V^{(2)}}{V^{(1)}}\right\}\right]=\mathbb{E}\left[\exp \left\{-\frac{t^{2} \sigma_{A}^{2}\left(1-\mu_{A}^{2}\right)^{2}}{2\left(1-\mu_{A}^{3}\right)} \frac{\sum_{i=1}^{\infty} P_{i}^{3}}{\left(\sum_{i=1}^{\infty} P_{i}^{2}\right)^{2}}\right\}\right] .
$$


To prove the existence of a uniformly continuous and continuously differentiable density function, it is enough to check that

$$
\int_{-\infty}^{\infty}|t| \mathbb{E}\left[\mathrm{e}^{-C_{U} t^{2} U / 2}\right] \mathrm{d} t=C_{U}^{-1} \int_{-\infty}^{\infty}|t| \mathbb{E}\left[\mathrm{e}^{-t^{2} U / 2}\right] \mathrm{d} t<\infty,
$$

where $U$ is given in (5.2) and

$$
C_{U}:=\frac{\theta^{-1 / \alpha} \sigma_{A}^{2}\left(1-\mu_{A}^{2}\right)^{2}}{1-\mu_{A}^{3}},
$$

see, e.g., Sato [38, Proposition 28.1]. Here, using Fubini's theorem,

$$
\begin{aligned}
\int_{-\infty}^{\infty}|t| \mathbb{E}\left[\mathrm{e}^{-t^{2} U / 2}\right] \mathrm{d} t & =\sqrt{2 \pi} \mathbb{E}\left[U^{-1 / 2} \int_{-\infty}^{\infty}|t| \frac{1}{\sqrt{2 \pi U^{-1}}} \mathrm{e}^{-t^{2} /\left(2 U^{-1}\right)} \mathrm{d} t\right] \\
& =\sqrt{2 \pi} \mathbb{E}\left[U^{-1 / 2} \mathbb{E}\left[U^{-1 / 2}|Z| \mid U\right]\right] \\
& =\sqrt{2 \pi} \mathbb{E}\left[U^{-1}|Z|\right]=\sqrt{2 \pi} \mathbb{E}\left[U^{-1}\right] \mathbb{E}[|Z|]
\end{aligned}
$$

where $Z$ is a standard normally distributed random variable independent of $U$. Thus we only have to show that $\mathbb{E}\left[U^{-1}\right]<\infty$, i.e., by $(5.2)$,

$$
\mathbb{E}\left[\frac{\left(\sum_{i=1}^{\infty} \Gamma_{i}^{-2 / \alpha}\right)^{2}}{\sum_{i=1}^{\infty} \Gamma_{i}^{-3 / \alpha}}\right]<\infty .
$$

In what follows we will use the following facts:

- $\left(\Gamma_{i}\right)_{i \geq 2} \stackrel{d}{=}\left(\Gamma_{1}+\Gamma_{i-1}^{\prime}\right)_{i \geq 2}$, where $\left(\Gamma_{i}^{\prime}\right)_{i \geq 1}$ has the same distribution as $\left(\Gamma_{i}\right)_{i \in \mathbb{N}}$ and independent of it,

- by Campbell's theorem,

$$
\mathbb{E}\left[\sum_{i=1}^{\infty} h\left(\Gamma_{i}\right)\right]=\mathbb{E}\left[\left(\sum_{i=1}^{\infty} \delta_{\Gamma_{i}}\right)(h)\right]=\int_{0}^{\infty} h(y) \mathrm{d} y
$$

for any Borel measurable function $h:(0, \infty) \rightarrow \mathbb{R}$ in the sense that the expectations exist on the left hand side if and only if the integral on the right hand side converges and then they are equal,

- if $\int_{0}^{\infty} h(x) \mathrm{d} x$ converges, then

$$
\mathbb{E}\left[\left(\sum_{i=1}^{\infty} h\left(\Gamma_{i}\right)\right)^{2}\right]=\int_{0}^{\infty} h^{2}(y) \mathrm{d} y+\left(\int_{0}^{\infty} h(y) \mathrm{d} y\right)^{2}
$$

where the right hand side can be finite or infinite as well. 
So, by conditioning on $\Gamma_{1}$ having an exponential distribution with parameter 1 , we obtain

$$
\begin{aligned}
& \mathbb{E}\left[\frac{\left(\sum_{i=1}^{\infty} \Gamma_{i}^{-2 / \alpha}\right)^{2}}{\sum_{i=1}^{\infty} \Gamma_{i}^{-3 / \alpha}}\right] \leq \mathbb{E}\left[\left(\sum_{i=1}^{\infty} \Gamma_{i}^{-2 / \alpha}\right)^{2} \Gamma_{1}^{3 / \alpha}\right] \\
& =\int_{0}^{\infty} x^{3 / \alpha} \mathrm{e}^{-x} \mathbb{E}\left[\left(x^{-2 / \alpha}+\sum_{i=1}^{\infty}\left(x+\Gamma_{i}^{\prime}\right)^{-2 / \alpha}\right)^{2}\right] \mathrm{d} x \\
& =\int_{0}^{\infty} x^{3 / \alpha} \mathrm{e}^{-x}\left(x^{-4 / \alpha}+2 x^{-2 / \alpha} \mathbb{E}\left[\sum_{i=1}^{\infty}\left(x+\Gamma_{i}^{\prime}\right)^{-2 / \alpha}\right]+\mathbb{E}\left[\left(\sum_{i=1}^{\infty}\left(x+\Gamma_{i}^{\prime}\right)^{-2 / \alpha}\right)^{2}\right]\right) \mathrm{d} x \\
& =\int_{0}^{\infty} x^{3 / \alpha} \mathrm{e}^{-x}\left(x^{-4 / \alpha}+2 x^{-2 / \alpha} \int_{x}^{\infty} y^{-2 / \alpha} \mathrm{d} y+\int_{x}^{\infty} y^{-4 / \alpha} \mathrm{d} y+\left(\int_{x}^{\infty} y^{-2 / \alpha} \mathrm{d} y\right)^{2}\right) \mathrm{d} x \\
& =\int_{0}^{\infty} \mathrm{e}^{-x}\left(x^{-1 / \alpha}+\left(\frac{2 \alpha}{2-\alpha}+\frac{\alpha}{4-\alpha}\right) x^{1-1 / \alpha}+\frac{\alpha^{2}}{(2-\alpha)^{2}} x^{2-1 / \alpha}\right) \mathrm{d} x<\infty,
\end{aligned}
$$

as desired, since $\int_{0}^{\infty} x^{n-\frac{1}{\alpha}} \mathrm{e}^{-x} \mathrm{~d} x=\Gamma\left(n+1-\frac{1}{\alpha}\right)<\infty, n \geq 0$.

Proposition 5.7. For each $x \in \mathbb{R}$, we have

$$
\mathbb{P}\left(\frac{V^{(2)}}{V^{(1)}} \leq x\right)=\frac{1}{2}-\frac{1}{2 \pi \mathrm{i}} \int_{-\infty}^{\infty} \frac{\varphi_{V^{(1)}, V^{(2)}}(-u x, u)}{u} \mathrm{~d} u
$$

where $\varphi_{V^{(1)}, V^{(2)}}$ denotes the joint characteristic function of $\left(V^{(1)}, V^{(2)}\right)$ given in Theorem 4.1, and $\int_{-\infty}^{\infty}$ is meant in the sense of Cauchy principal value, i.e., $\int_{-\infty}^{\infty}:=\lim _{T \rightarrow \infty} \lim _{h \rightarrow 0}\left(\int_{h}^{T}+\int_{-T}^{-h}\right)$.

Proof. By Proposition 5.6, $V^{(2)} / V^{(1)}$ is absolutely continuous, so the inversion formula for characteristic functions due to Gurland [16] yields that for each $x \in \mathbb{R}$,

$$
\mathbb{P}\left(\frac{V^{(2)}}{V^{(1)}} \leq x\right)=\mathbb{P}\left(V^{(2)}-x V^{(1)} \leq 0\right)=\frac{1}{2}-\frac{1}{2 \pi \mathrm{i}} \int_{-\infty}^{\infty} \mathrm{e}^{-\mathrm{i} u 0} \frac{\varphi_{V^{(2)}-x V^{(1)}}(u)}{u} \mathrm{~d} u,
$$

yielding the statement, where $\varphi_{V^{(2)}-x V^{(1)}}$ denotes the characteristic function of $V^{(2)}-x V^{(1)}$.

\section{A On topological properties of $S$}

Lemma A.1. The set $S=(0, \infty) \times \mathbb{R}$ furnished with the metric d given in $(3.3)$ is a complete separable metric space, and $B \subset S$ is bounded with respect to the metric $d$ if and only if $B$ is separated from the vertical line $\{(0, y): y \in \mathbb{R}\}$, i.e., there exists $\epsilon>0$ such that $B \subset$ $\{(x, y) \in S: x>\epsilon\}$. Moreover, the topology and the Borel $\sigma$-algebra $\mathcal{B}(S)$ on $S$ induced by the metric $d$ coincides with the topology and the Borel $\sigma$-algebra on $S$ induced by the usual metric $\rho\left(\left(x, x^{\prime}\right),\left(y, y^{\prime}\right)\right):=\sqrt{\left(x-x^{\prime}\right)^{2}+\left(y-y^{\prime}\right)^{2}},\left(x, x^{\prime}\right),\left(y, y^{\prime}\right) \in S$, respectively. 
Proof. First, we check that $S$ is a complete separable metric space. If $\left(x_{n}, y_{n}\right)_{n \in \mathbb{N}}$ is a Cauchy sequence in $S$, then for all $\epsilon \in(0,1)$, there exists an $N_{\epsilon} \in \mathbb{N}$ such that $d\left(\left(x_{n}, y_{n}\right),\left(x_{m}, y_{m}\right)\right)<\epsilon$ for $n, m \geq N_{\epsilon}$. Hence $\rho\left(\left(x_{n}, y_{n}\right),\left(x_{m}, y_{m}\right)\right)<\epsilon$ and $\left|\frac{1}{x_{n}}-\frac{1}{x_{m}}\right|<\epsilon$ for $n, m \geq N_{\epsilon}$, i.e., $\left(x_{n}, y_{n}\right)_{n \in \mathbb{N}}$ and $\left(1 / x_{n}\right)_{n \in \mathbb{N}}$ are Cauchy sequences in $\mathbb{R}^{2}$ and in $\mathbb{R}$, respectively. Consequently, there exists an $(x, y) \in[0, \infty) \times \mathbb{R}$ such that $\lim _{n \rightarrow \infty}\left(x_{n}, y_{n}\right)=(x, y)$ and $\frac{1}{x_{n}}$ being convergent as $n \rightarrow \infty$, yielding that $x>0$, and so $(x, y) \in(0, \infty) \times \mathbb{R}$. By continuity, $\lim _{n \rightarrow \infty} d\left(\left(x_{n}, y_{n}\right),(x, y)\right)=0$, as desired. The separability of $S$ readily follows, since $S \cap \mathbb{Q}^{2}$ is a countable everywhere dense subset of $S$.

Next, we check that $B \subset S$ is bounded with respect to the metric $d$ if and only if there exists $\epsilon>0$ such that $B \subset\{(x, y) \in S: x>\epsilon\}$. If $B \subset S$ is bounded, then there exists $r>0$ such that $d((x, y),(1,0))<r,(x, y) \in B$, yielding that $\left|\frac{1}{x}-1\right|<r,(x, y) \in B$, and then $x>\frac{1}{1+r}$, $(x, y) \in B$, so one can choose $\epsilon=\frac{1}{1+r}$. If there exists $\epsilon>0$ such that $B \subset\{(x, y) \in S: x>\epsilon\}$, then $d((x, y),(1,0))=\min \left(\sqrt{(x-1)^{2}+y^{2}}, 1\right)+\left|\frac{1}{x}-1\right| \leq 1+\frac{1}{\epsilon}+1,(x, y) \in B$.

Since $S$ is locally compact, second countable and Hausdorff, one could choose a metric such that the relatively compact sets are precisely the bounded ones, see Kallenberg [21, page 18]. The metric $d$ does not have this property, but we do not need it. For historical fidelity, we note that originally the vague convergence of point measures in $\mathcal{M}_{p}(S)$ is defined by the convergence of integrals of some compactly supported functions (see, e.g., Resnick [36, Section 3.3.5]), but recently instead of compactly supported functions one uses functions with bounded support (see, e.g., Kallenberg [21]). We also follow the latter approach.

\section{B Vague convergence of point measures}

Proof of Lemma 3.1. First, let us suppose that $\mu_{n} \stackrel{v}{\longrightarrow} \mu$ as $n \rightarrow \infty$, and let $\epsilon>0$ be such that $\mu(\{\epsilon\} \times \mathbb{R})=0$. Since $\mu$ is locally finite and $(\epsilon, \infty) \times \mathbb{R}$ is bounded, there exist integers $K \geq 0$ and $c_{1}, \ldots, c_{K} \in \mathbb{N}$ such that

$$
\left.\mu\right|_{(\epsilon, \infty) \times \mathbb{R}}=\sum_{j=1}^{K} c_{j} \delta_{\left(u_{j}, v_{j}\right)},
$$

where $\left(u_{1}, v_{1}\right), \ldots,\left(u_{K}, v_{K}\right)$ are the atoms of $\mu$ in $(\epsilon, \infty) \times \mathbb{R}$ and $c_{1}, \ldots, c_{K}$ are their multiplicities. Let $s_{0}:=0, s_{j}:=c_{1}+\cdots+c_{j}$ for $j \in\{1, \ldots, K\}$ and $M:=s_{K}$, and let us label the points of $\left.\mu\right|_{(\epsilon, \infty) \times \mathbb{R}}$ such that for each $j \in\{1, \ldots, K\}$ we have $\left(u_{j}, v_{j}\right)=\left(x_{k}, y_{k}\right)$ for all $k \in\left\{s_{j-1}+1, \ldots, s_{j}\right\}$, yielding that $\left.\mu\right|_{(\epsilon, \infty) \times \mathbb{R}}=\sum_{i=1}^{M} \delta_{\left(x_{i}, y_{i}\right)}$. Since $(\epsilon, \infty) \times \mathbb{R}$ is open in $S$ (see Lemma A.1), one can choose pairwise disjoint open sets $G_{1}, \ldots, G_{K} \subset(\epsilon, \infty) \times \mathbb{R}$ such that $\left(u_{j}, v_{j}\right) \in G_{j}, j=1, \ldots K$. Especially, we have $\mu\left(\partial G_{j}\right)=0, j \in\{1, \ldots, K\}$, where $\partial G_{j}$ denotes the boundary of $G_{j}$ in $S$ ( since $\left.\mu\right|_{(\epsilon, \infty) \times \mathbb{R}}$ puts zero mass outside the points $\left.\left(u_{1}, v_{1}\right), \ldots,\left(u_{K}, v_{K}\right)\right)$. Hence, since $\mu_{n} \stackrel{v}{\longrightarrow} \mu$ as $n \rightarrow \infty$, using the equivalence of (i) and (iv) in Lemma 4.1 in Kallenberg [21], we have $\mu_{n}\left(G_{j}\right) \rightarrow \mu\left(G_{j}\right)=c_{j}$ as $n \rightarrow \infty$ for each $j \in\{1, \ldots, K\}$. Similarly,

$$
\mu_{n}\left(((\epsilon, \infty) \times \mathbb{R}) \backslash\left(G_{1} \cup \cdots \cup G_{K}\right)\right) \rightarrow \mu\left(((\epsilon, \infty) \times \mathbb{R}) \backslash\left(G_{1} \cup \cdots \cup G_{K}\right)\right)=0 \quad \text { as } n \rightarrow \infty,
$$


since $((\epsilon, \infty) \times \mathbb{R}) \backslash\left(G_{1} \cup \cdots \cup G_{K}\right)$ is a bounded (with respect to metric $d$ given in (3.3)) Borel subset of $S$ and $\left.\mu\left(\partial((\epsilon, \infty) \times \mathbb{R}) \backslash\left(G_{1} \cup \cdots \cup G_{K}\right)\right)\right)=0$ (using also the assumption $\mu(\{\epsilon\} \times \mathbb{R})=$ $0)$. Consequently, since $\mu_{n}, n \in \mathbb{N}$, and $\mu$ are integer-valued measures, there exists an integer $n_{0} \geq 0$ such that $\mu_{n}\left(G_{j}\right)=c_{j}, j \in\{1, \ldots, K\}$, and $\mu_{n}\left(((\epsilon, \infty) \times \mathbb{R}) \backslash\left(G_{1} \cup \cdots \cup G_{K}\right)\right)=0$ for all $n \geq n_{0}$, yielding that $\mu_{n}((\epsilon, \infty) \times \mathbb{R})=\mu((\epsilon, \infty) \times \mathbb{R})=c_{1}+\cdots+c_{K}=M$ for all $n \geq n_{0}$. So for each $n \geq n_{0}$, one can label the points of $\left.\mu_{n}\right|_{(\epsilon, \infty) \times \mathbb{R}}$ such that for each $j \in\{1, \ldots, K\}$ we have $\left(x_{k}^{(n)}, y_{k}^{(n)}\right) \in G_{j}$ for all $k \in\left\{s_{j-1}+1, \ldots, s_{j}\right\}$, yielding that $\left.\mu_{n}\right|_{(\epsilon, \infty) \times \mathbb{R}}=\sum_{i=1}^{M} \delta_{\left(x_{i}^{(n)}, y_{i}^{(n)}\right)}$. Shrinking the open sets $G_{1}, \ldots, G_{K}$ onto $\left(u_{1}, v_{1}\right), \ldots,\left(u_{K}, v_{K}\right)$, respectively, we have $x_{i}^{(n)} \rightarrow x_{i}$ and $y_{i}^{(n)} \rightarrow y_{i}$ as $n \rightarrow \infty$ for all $i=1, \ldots, M$.

Now, let us prove the reverse direction. Let us suppose that $f: S \rightarrow[0, \infty)$ is a bounded, continuous function with bounded support. Then, using Lemma A.1, there exists $\epsilon_{0} \in(0, \infty)$ such that $f(x, y)=0$ for all $(x, y) \in\left(0, \epsilon_{0}\right] \times \mathbb{R}$. Since the function $\left(0, \epsilon_{0}\right) \ni \epsilon \mapsto \mu((\epsilon, \infty) \times \mathbb{R}) \in$ $[0, \infty)$ is decreasing, there exists $\epsilon \in\left(0, \epsilon_{0}\right)$ such that $\mu(\{\epsilon\} \times \mathbb{R})=0$. Due to our assumptions, there exist integers $n_{0}, M \geq 0$ and a labeling of the points of $\mu$ and $\mu_{n}, n \geq n_{0}$, in $(\epsilon, \infty) \times \mathbb{R}$ such that

$$
\left.\mu_{n}\right|_{(\epsilon, \infty) \times \mathbb{R}}=\sum_{i=1}^{M} \delta_{\left(x_{i}^{(n)}, y_{i}^{(n)}\right)},\left.\quad \mu\right|_{(\epsilon, \infty) \times \mathbb{R}}=\sum_{i=1}^{M} \delta_{\left(x_{i}, y_{i}\right)},
$$

and $x_{i}^{(n)} \rightarrow x_{i}$ and $y_{i}^{(n)} \rightarrow y_{i}$ as $n \rightarrow \infty$ for all $i=1, \ldots, M$. Consequently,

$$
\begin{aligned}
\mu_{n}(f) & =\iint_{\left(\epsilon_{0}, \infty\right) \times \mathbb{R}} f(x, y) \mu_{n}(\mathrm{~d} x, \mathrm{~d} y)=\iint_{(\epsilon, \infty) \times \mathbb{R}} f(x, y) \mu_{n}(\mathrm{~d} x, \mathrm{~d} y)=\sum_{i=1}^{M} f\left(x_{i}^{(n)}, y_{i}^{(n)}\right) \\
& \rightarrow \sum_{i=1}^{M} f\left(x_{i}, y_{i}\right)=\iint_{(\epsilon, \infty) \times \mathbb{R}} f(x, y) \mu(\mathrm{d} x, \mathrm{~d} y)=\iint_{\left(\epsilon_{0}, \infty\right) \times \mathbb{R}} f(x, y) \mu(\mathrm{d} x, \mathrm{~d} y)=\mu(f)
\end{aligned}
$$

as $n \rightarrow \infty$, hence we have $\mu_{n} \stackrel{v}{\longrightarrow} \mu$ as $n \rightarrow \infty$, as desired.

\section{Approximation of Laplace functionals}

First, we recall an auxiliary lemma stating that $\left(X_{i}\right)_{i \geq 0}$ is strongly mixing with a geometric rate from Basrak et al. [5, Remark 3.1] and Barczy et al. [3, Lemma F.1].

A strongly stationary sequence $\left(Y_{k}\right)_{k \geq 0}$ is called strongly mixing with a rate function $\left(\alpha_{h}\right)_{h \in \mathbb{N}}$ if its strongly stationary extensions $\left(Y_{k}\right)_{k \in \mathbb{Z}}$ admit this property, namely,

$$
\alpha_{h}:=\sup _{A \in \mathcal{F}_{-\infty, 0}^{Y}, B \in \mathcal{F}_{h, \infty}^{Y}}|\mathbb{P}(A \cap B)-\mathbb{P}(A) \mathbb{P}(B)| \rightarrow 0 \quad \text { as } h \rightarrow \infty,
$$

where $\mathcal{F}_{-\infty, 0}^{Y}:=\sigma\left(\ldots, Y_{-1}, Y_{0}\right), \mathcal{F}_{h, \infty}^{Y}:=\sigma\left(Y_{h}, Y_{h+1}, \ldots\right), h \in \mathbb{N}$.

Lemma C.1. The strongly stationary Markov chain $\left(X_{i}\right)_{i \geq 0}$ is strongly mixing with a geometric rate, i.e., there exists a constant $q \in(0,1)$ such that $\alpha_{\ell}=O\left(q^{\ell}\right)$ as $\ell \rightarrow \infty$. 
Note that in this paper we need only that $\left(X_{i}\right)_{i \geq 0}$ is strongly mixing, and we will not use that the mixing rate is geometric, however, for completeness, we decided to recall it in Lemma C.1 as well.

Next, we show that the process $\left(X_{i} \mathbb{1}_{\left\{X_{i}>0\right\}}, \frac{M_{i+1}}{\sqrt{X_{i}}} \mathbb{1}_{\left\{X_{i}>0\right\}}\right)_{i \geq 0}$ satisfies a certain mixing condition (for the definition of $M_{i+1}, i \geq 0$, see the Introduction).

Lemma C.2. There exists a sequence of positive integers $\left(r_{n}\right)_{n \in \mathbb{N}}$ with $r_{n} \rightarrow \infty$ and $r_{n} / n \rightarrow 0$ as $n \rightarrow \infty$ such that for each bounded, continuous function $f: S \rightarrow[0, \infty)$ having the property $f(x, y)=0$ for all $(x, y) \in(0, \epsilon] \times \mathbb{R}$ for some $\epsilon>0$, we have

$$
\mathbb{E}\left[\exp \left\{-\sum_{i=1}^{n} f\left(\frac{X_{i}}{a_{n}}, \frac{M_{i+1}}{\sqrt{X_{i}}}\right)\right\}\right]-\left(\mathbb{E}\left[\exp \left\{-\sum_{i=1}^{r_{n}} f\left(\frac{X_{i}}{a_{n}}, \frac{M_{i+1}}{\sqrt{X_{i}}}\right)\right\}\right]\right)^{k_{n}} \rightarrow 0
$$

as $n \rightarrow \infty$ with $k_{n}:=\left\lfloor n / r_{n}\right\rfloor$, where we recall that $S=(0, \infty) \times \mathbb{R}$ and

$$
\sum_{i=1}^{m}=\sum_{\left\{j \in\{1, \ldots, m\}: X_{j}>0\right\}}, \quad m \in \mathbb{N} .
$$

Proof. We follow the proof of Proposition 1.34 in Krizmanić [25] (see also Basrak [4, Lemma 2.3.9]). Let $\left(\ell_{n}\right)_{n \in \mathbb{N}}$ be a sequence of positive integers with $\ell_{n} \rightarrow \infty$ and $\ell_{n} / n^{1 / 8} \rightarrow 0$ as $n \rightarrow \infty$. We will show that the sequence

$$
r_{n}:=\left\lfloor\max \left\{n \sqrt{\alpha_{\ell_{n}}}, n^{2 / 3}\right\}\right\rfloor+1, \quad n \in \mathbb{N},
$$

is a good choice with $\alpha_{\ell}, \ell \in \mathbb{N}$, given in (C.1). Clearly, $r_{n} \rightarrow \infty$ as $n \rightarrow \infty$. By Lemma C.1, the strongly stationary Markov chain $\left(X_{i}\right)_{i \geq 0}$ is strongly mixing, i.e., $\alpha_{\ell_{n}} \rightarrow 0$ as $n \rightarrow \infty$, yielding $r_{n} / n \rightarrow 0$ as $n \rightarrow \infty$ and

$$
k_{n} \rightarrow \infty, \quad k_{n} \alpha_{\ell_{n}} \rightarrow 0, \quad \frac{k_{n} \ell_{n}}{n} \rightarrow 0
$$

as $n \rightarrow \infty$.

Fix a bounded, continuous function $f: S \rightarrow[0, \infty)$ having the property $f(x, y)=0$ for all $(x, y) \in(0, \epsilon] \times \mathbb{R}$ for some $\epsilon>0$. Put $M:=\sup _{(x, y) \in S} f(x, y)<\infty$. We have to show that $I(n) \rightarrow 0$ as $n \rightarrow \infty$ with

$$
I(n):=\left|\mathbb{E}\left[\exp \left\{-\sum_{i=1}^{n} f\left(\frac{X_{i}}{a_{n}}, \frac{M_{i+1}}{\sqrt{X_{i}}}\right)\right\}\right]-\left(\mathbb{E}\left[\exp \left\{-\sum_{i=1}^{r_{n}} f\left(\frac{X_{i}}{a_{n}}, \frac{M_{i+1}}{\sqrt{X_{i}}}\right)\right\}\right]\right)^{k_{n}}\right| .
$$

We have

$$
I(n) \leq I_{1}(n)+I_{2}(n)+I_{3}(n)+I_{4}(n), \quad n \in \mathbb{N},
$$


with

$$
\begin{aligned}
& I_{1}(n):=\left|\mathbb{E}\left[\exp \left\{-\sum_{i=1}^{n} f\left(\frac{X_{i}}{a_{n}}, \frac{M_{i+1}}{\sqrt{X_{i}}}\right)\right\}\right]-\mathbb{E}\left[\exp \left\{-\sum_{i=1}^{k_{n} r_{n}} f\left(\frac{X_{i}}{a_{n}}, \frac{M_{i+1}}{\sqrt{X_{i}}}\right)\right\}\right]\right|, \\
& I_{2}(n):=\mid \mathbb{E}\left[\exp \left\{-\sum_{i=1}^{k_{n} r_{n}} f\left(\frac{X_{i}}{a_{n}}, \frac{M_{i+1}}{\sqrt{X_{i}}}\right)\right\}\right]-\mathbb{E}\left[\exp \left\{-\sum_{k=1}^{k_{n}} \sum_{i=(k-1) r_{n}+1}^{k r_{n}-\ell_{n}} f\left(\frac{X_{i}}{a_{n}}, \frac{M_{i+1}}{\sqrt{X_{i}}}\right)\right\}\right], \\
& I_{3}(n):=\left|\mathbb{E}\left[\exp \left\{-\sum_{k=1}^{k_{n}} \sum_{i=(k-1) r_{n}+1}^{k r_{n}-\ell_{n}} f\left(\frac{X_{i}}{a_{n}}, \frac{M_{i+1}}{\sqrt{X_{i}}}\right)\right\}\right]-\left(\mathbb{E}\left[\exp \left\{-\sum_{i=1}^{r_{n}-\ell_{n}} f\left(\frac{X_{i}}{a_{n}}, \frac{M_{i+1}}{\sqrt{X_{i}}}\right)\right\}\right]\right)^{k_{n}}\right|, \\
& I_{4}(n):=\left|\left(\mathbb{E}\left[\exp \left\{-\sum_{i=1}^{r_{n}-\ell_{n}} f\left(\frac{X_{i}}{a_{n}}, \frac{M_{i+1}}{\sqrt{X_{i}}}\right)\right\}\right]\right)^{k_{n}}-\left(\mathbb{E}\left[\exp \left\{-\sum_{i=1}^{r_{n}} f\left(\frac{X_{i}}{a_{n}}, \frac{M_{i+1}}{\sqrt{X_{i}}}\right)\right\}\right]\right)^{k_{n}}\right|,
\end{aligned}
$$

where, by (C.2), $k r_{n}-\ell_{n} \rightarrow \infty$ as $n \rightarrow \infty$ for each $k \in \mathbb{N}$. By the strong stationarity of $\left(X_{k}\right)_{k \geq 0}$ and using the inequality $1-\mathrm{e}^{-x} \leq x$ for any $x \in(0, \infty)$, we obtain

$$
\begin{aligned}
I_{1}(n) & \leq \mathbb{E}\left[\exp \left\{-\sum_{i=1}^{k_{n} r_{n}} f\left(\frac{X_{i}}{a_{n}}, \frac{M_{i+1}}{\sqrt{X_{i}}}\right)\right\}\left|1-\exp \left\{-\sum_{i=k_{n} r_{n}+1}^{n} f\left(\frac{X_{i}}{a_{n}}, \frac{M_{i+1}}{\sqrt{X_{i}}}\right)\right\}\right|\right] \\
& \leq \mathbb{E}\left[\sum_{i=k_{n} r_{n}+1}^{n} f\left(\frac{X_{i}}{a_{n}}, \frac{M_{i+1}}{\sqrt{X_{i}}}\right)\right]=\sum_{i=k_{n} r_{n}+1}^{n} \mathbb{E}\left[f\left(\frac{X_{i}}{a_{n}}, \frac{M_{i+1}}{\sqrt{X_{i}}}\right) \mathbb{1}_{\left\{\frac{X_{i}}{a_{n}}>\epsilon\right\}}\right] \\
& \leq \sum_{i=k_{n} r_{n}+1}^{n} M \mathbb{P}\left(X_{i}>\epsilon a_{n}\right)=\left(n-k_{n} r_{n}\right) M \mathbb{P}\left(X_{0}>\epsilon a_{n}\right) .
\end{aligned}
$$

In a similar manner we obtain

$$
\begin{aligned}
I_{2}(n) & \leq \mathbb{E}\left[\exp \left\{-\sum_{k=1}^{k_{n}} \sum_{i=(k-1) r_{n}+1}^{k r_{n}-\ell_{n}} f\left(\frac{X_{i}}{a_{n}}, \frac{M_{i+1}}{\sqrt{X_{i}}}\right)\right\}\left|1-\exp \left\{-\sum_{k=1}^{k_{n}} \sum_{i=k r_{n}-\ell_{n}+1}^{k r_{n}} f\left(\frac{X_{i}}{a_{n}}, \frac{M_{i+1}}{\sqrt{X_{i}}}\right)\right\}\right|\right] \\
& \leq \mathbb{E}\left[\sum_{k=1}^{k_{n}} \sum_{i=k r_{n}-\ell_{n}+1}^{k r_{n}} f\left(\frac{X_{i}}{a_{n}}, \frac{M_{i+1}}{\sqrt{X_{i}}}\right)\right]=\sum_{k=1}^{k_{n}} \sum_{i=k r_{n}-\ell_{n}+1}^{k r_{n}} \mathbb{E}\left[f\left(\frac{X_{i}}{a_{n}}, \frac{M_{i+1}}{\sqrt{X_{i}}}\right) \mathbb{1}_{\left\{\frac{X_{i}}{a_{n}}>\epsilon\right\}}\right] \\
& \leq \sum_{k=1}^{k_{n}} \sum_{i=k r_{n}-\ell_{n}+1}^{k r_{n}} M \mathbb{P}\left(X_{i}>\epsilon a_{n}\right)=k_{n} \ell_{n} M \mathbb{P}\left(X_{0}>\epsilon a_{n}\right) .
\end{aligned}
$$

We have

$$
I_{3}(n) \leq I_{5}(n)+I_{6}(n), \quad n \in \mathbb{N},
$$


with

$$
\begin{aligned}
& I_{5}(n):=\mid \mathbb{E}\left[\exp \left\{-\sum_{k=1}^{k_{n}} \sum_{i=(k-1) r_{n}+1}^{k r_{n}-\ell_{n}} f\left(\frac{X_{i}}{a_{n}}, \frac{M_{i+1}}{\sqrt{X_{i}}}\right)\right\}\right] \\
&-\mathbb{E}\left[\exp \left\{-\sum_{i=1}^{r_{n}-\ell_{n}} f\left(\frac{X_{i}}{a_{n}}, \frac{M_{i+1}}{\sqrt{X_{i}}}\right)\right\}\right] \mathbb{E}\left[\exp \left\{-\sum_{k=2}^{k_{n}} \sum_{i=(k-1) r_{n}+1}^{k r_{n}-\ell_{n}} f\left(\frac{X_{i}}{a_{n}}, \frac{M_{i+1}}{\sqrt{X_{i}}}\right)\right\}\right], \\
& I_{6}(n):=\mid \mathbb{E}\left[\exp \left\{-\sum_{i=1}^{r_{n}-\ell_{n}} f\left(\frac{X_{i}}{a_{n}}, \frac{M_{i+1}}{\sqrt{X_{i}}}\right)\right\}\right] \mathbb{E}\left[\exp \left\{-\sum_{k=2}^{k_{n}} \sum_{i=(k-1) r_{n}+1}^{k r_{n}-\ell_{n}} f\left(\frac{X_{i}}{a_{n}}, \frac{M_{i+1}}{\sqrt{X_{i}}}\right)\right\}\right] \\
&-\left(\mathbb{E}\left[\exp \left\{-\sum_{i=1}^{r_{n}-\ell_{n}} f\left(\frac{X_{i}}{a_{n}}, \frac{M_{i+1}}{\sqrt{X_{i}}}\right)\right\}\right]\right)^{k_{n}} \mid .
\end{aligned}
$$

Since $\left(X_{i}\right)_{i \geq 0}$ is strongly mixing, we have

$$
|\mathbb{E}[\xi \eta]-\mathbb{E}[\xi] \mathbb{E}[\eta]| \leq 4 C_{1} C_{2} \alpha_{m}
$$

for any $\mathcal{F}_{0, j}^{X}$-measurable random variable $\xi$ and any $\mathcal{F}_{j+m, \infty}^{X}$-measurable random variable $\eta$ with $j, m \in \mathbb{N},|\xi| \leq C_{1}$ and $|\eta| \leq C_{2}$ (see, e.g., Lemma 1.2.1 in Lin and Lu [30]). Hence, using that the random variables

$$
\sum_{i=1}^{r_{n}-\ell_{n}} f\left(\frac{X_{i}}{a_{n}}, \frac{M_{i+1}}{\sqrt{X_{i}}}\right) \quad \text { and } \quad \sum_{k=2}^{k_{n}} \sum_{i=(k-1) r_{n}+1}^{k r_{n}-\ell_{n}} f\left(\frac{X_{i}}{a_{n}}, \frac{M_{i+1}}{\sqrt{X_{i}}}\right)
$$

are $\mathcal{F}_{0, r_{n}-\ell_{n}+1}^{X}$-measurable and $\mathcal{F}_{r_{n}+1, \infty}^{X}$-measurable, respectively, we have

$$
I_{5}(n) \leq 4 \alpha_{\ell_{n}}, \quad n \in \mathbb{N} .
$$

It is easy to obtain that

$$
\begin{aligned}
& I_{6}(n)=\mathbb{E}\left[\exp \left\{-\sum_{i=1}^{r_{n}-\ell_{n}} f\left(\frac{X_{i}}{a_{n}}, \frac{M_{i+1}}{\sqrt{X_{i}}}\right)\right\}\right] \\
& \times\left|\mathbb{E}\left[\exp \left\{-\sum_{k=2}^{k_{n}} \sum_{i=(k-1) r_{n}+1}^{k r_{n}-\ell_{n}} f\left(\frac{X_{i}}{a_{n}}, \frac{M_{i+1}}{\sqrt{X_{i}}}\right)\right\}\right]-\left(\mathbb{E}\left[\exp \left\{-\sum_{i=1}^{r_{n}-\ell_{n}} f\left(\frac{X_{i}}{a_{n}}, \frac{M_{i+1}}{\sqrt{X_{i}}}\right)\right\}\right]\right)^{k_{n}-1}\right| \\
& \leq\left|\mathbb{E}\left[\exp \left\{-\sum_{k=2}^{k_{n}} \sum_{i=(k-1) r_{n}+1}^{k r_{n}-\ell_{n}} f\left(\frac{X_{i}}{a_{n}}, \frac{M_{i+1}}{\sqrt{X_{i}}}\right)\right\}\right]-\left(\mathbb{E}\left[\exp \left\{-\sum_{i=1}^{r_{n}-\ell_{n}} f\left(\frac{X_{i}}{a_{n}}, \frac{M_{i+1}}{\sqrt{X_{i}}}\right)\right\}\right]\right)^{k_{n}-1}\right|
\end{aligned}
$$

hence we recursively obtain (we repeat the same procedure for the above estimation of $I_{6}(n)$ as we did for $I_{3}(n)$ and so on)

$$
I_{3}(n) \leq 4 k_{n} \alpha_{\ell_{n}}
$$


Strong stationarity of $\left(X_{i}\right)_{i \geq 0}$ and Lemma 4.3 in Chapter 2 in Durrett [14] imply

$$
\begin{aligned}
I_{4}(n) & \leq k_{n}\left|\mathbb{E}\left[\exp \left\{-\sum_{i=1}^{r_{n}-\ell_{n}} f\left(\frac{X_{i}}{a_{n}}, \frac{M_{i+1}}{\sqrt{X_{i}}}\right)\right\}\right]-\mathbb{E}\left[\exp \left\{-\sum_{i=1}^{r_{n}} *\left(\frac{X_{i}}{a_{n}}, \frac{M_{i+1}}{\sqrt{X_{i}}}\right)\right\}\right]\right| \\
& \leq k_{n} \mathbb{E}\left[\exp \left\{-\sum_{i=1}^{r_{n}-\ell_{n}} f\left(\frac{X_{i}}{a_{n}}, \frac{M_{i+1}}{\sqrt{X_{i}}}\right)\right\}\left|1-\exp \left\{-\sum_{i=r_{n}-\ell_{n}+1}^{r_{n}} f\left(\frac{X_{i}}{a_{n}}, \frac{M_{i+1}}{\sqrt{X_{i}}}\right)\right\}\right|\right] \\
& \leq k_{n} \mathbb{E}\left[\sum_{i=r_{n}-\ell_{n}+1}^{r_{n}} f\left(\frac{X_{i}}{a_{n}}, \frac{M_{i+1}}{\sqrt{X_{i}}}\right)\right]=k_{n} \sum_{i=r_{n}-\ell_{n}+1}^{r_{n}} \mathbb{E}\left[f\left(\frac{X_{i}}{a_{n}}, \frac{M_{i+1}}{\sqrt{X_{i}}}\right) \mathbb{1}_{\left\{\frac{X_{i}}{a_{n}}>\epsilon\right\}}\right] \\
& \leq k_{n} \sum_{i=r_{n}-\ell_{n}+1}^{r_{n}} M \mathbb{P}\left(X_{i}>\epsilon a_{n}\right)=k_{n} \ell_{n} M \mathbb{P}\left(X_{0}>\epsilon a_{n}\right) .
\end{aligned}
$$

Since $X_{0}$ is regularly varying with tail index $\alpha$, by (3.1) and (C.2), we obtain

$$
\begin{aligned}
I(n) & \leq\left(n-k_{n} r_{n}+2 k_{n} \ell_{n}\right) M \mathbb{P}\left(X_{0}>\epsilon a_{n}\right)+4 k_{n} \alpha_{\ell_{n}} \\
& =\frac{\left(n-k_{n} r_{n}+2 k_{n} \ell_{n}\right) M}{n} \cdot n \mathbb{P}\left(X_{0}>a_{n}\right) \cdot \frac{\mathbb{P}\left(X_{0}>\epsilon a_{n}\right)}{\mathbb{P}\left(X_{0}>a_{n}\right)}+4 k_{n} \alpha_{\ell_{n}} \rightarrow 0
\end{aligned}
$$

as $n \rightarrow \infty$, since

$$
\frac{n-k_{n} r_{n}}{n} \leq \frac{n-\left(\frac{n}{r_{n}}-1\right) r_{n}}{n}=\frac{r_{n}}{n} \rightarrow 0 \quad \text { as } n \rightarrow \infty
$$

as desired.

\section{Conditional Slutsky's lemma, conditional continuous mapping theorem}

First, we prove the analogues of parts (iv) and (v) of Theorem 2.7 in van der Vaart [42] for probability measures instead of random vectors.

Lemma D.1. Let $\mu_{n}, n \in \mathbb{N}$, be probability measures on $\left(\mathbb{R}^{2 k}, \mathcal{B}\left(\mathbb{R}^{2 k}\right)\right)$ with some $k \in \mathbb{N}$. For each $n \in \mathbb{N}$, consider the marginal probability measures $\mu_{n}^{(1)}$ and $\mu_{n}^{(2)}$ on $\left(\mathbb{R}^{k}, \mathcal{B}\left(\mathbb{R}^{k}\right)\right)$ defined by $\mu_{n}^{(1)}(B):=\mu_{n}\left(B \times \mathbb{R}^{k}\right)$ and $\mu_{n}^{(2)}(B):=\mu_{n}\left(\mathbb{R}^{k} \times B\right)$ for $B \in \mathcal{B}\left(\mathbb{R}^{k}\right)$. If $\mu_{n}^{(1)} \stackrel{w}{\longrightarrow} \mu^{(1)}$ as $n \rightarrow \infty$ with some probability measure $\mu^{(1)}$ on $\left(\mathbb{R}^{k}, \mathcal{B}\left(\mathbb{R}^{k}\right)\right)$ and $\mu_{n}\left(\left\{(\boldsymbol{x}, \boldsymbol{y}) \in \mathbb{R}^{k} \times \mathbb{R}^{k}:\|\boldsymbol{x}-\boldsymbol{y}\|>\epsilon\right\}\right) \rightarrow 0$ as $n \rightarrow \infty$ for all $\epsilon \in(0, \infty)$, then $\mu_{n}^{(2)} \stackrel{w}{\longrightarrow} \mu^{(1)}$ as $n \rightarrow \infty$.

Proof. For each bounded Lipschitz function $g: \mathbb{R}^{k} \rightarrow \mathbb{R}$ and for each $n \in \mathbb{N}$, we have

$$
\Delta_{n}^{(g)}:=\left|\int_{\mathbb{R}^{k}} g(\boldsymbol{y}) \mu_{n}^{(2)}(\mathrm{d} \boldsymbol{y})-\int_{\mathbb{R}^{k}} g(\boldsymbol{x}) \mu^{(1)}(\mathrm{d} \boldsymbol{x})\right| \leq I_{n}^{(g)}+J_{n}^{(g)},
$$


where

$$
\begin{aligned}
I_{n}^{(g)} & :=\left|\int_{\mathbb{R}^{k}} g(\boldsymbol{y}) \mu_{n}^{(2)}(\mathrm{d} \boldsymbol{y})-\int_{\mathbb{R}^{k}} g(\boldsymbol{x}) \mu_{n}^{(1)}(\mathrm{d} \boldsymbol{x})\right| \\
J_{n}^{(g)} & :=\left|\int_{\mathbb{R}^{k}} g(\boldsymbol{x}) \mu_{n}^{(1)}(\mathrm{d} \boldsymbol{x})-\int_{\mathbb{R}^{k}} g(\boldsymbol{x}) \mu^{(1)}(\mathrm{d} \boldsymbol{x})\right| .
\end{aligned}
$$

By the portmanteau lemma (see, e.g., van der Vaart [42, Lemma 2.2]), the convergence $\mu_{n}^{(1)} \stackrel{w}{\longrightarrow}$ $\mu^{(1)}$ as $n \rightarrow \infty$ implies $J_{n}^{(g)} \rightarrow 0$ as $n \rightarrow \infty$. Moreover, for each $\epsilon \in(0, \infty)$, by Fubini's theorem, we have

$$
\begin{aligned}
I_{n}^{(g)} & =\left|\int_{\mathbb{R}^{2 k}} g(\boldsymbol{y}) \mu_{n}(\mathrm{~d} \boldsymbol{x}, \mathrm{d} \boldsymbol{y})-\int_{\mathbb{R}^{2 k}} g(\boldsymbol{x}) \mu_{n}(\mathrm{~d} \boldsymbol{x}, \mathrm{d} \boldsymbol{y})\right| \\
& \leq \int_{\mathbb{R}^{2 k}}|g(\boldsymbol{y})-g(\boldsymbol{x})| \mu_{n}(\mathrm{~d} \boldsymbol{x}, \mathrm{d} \boldsymbol{y}) \\
& =\int_{\|\boldsymbol{x}-\boldsymbol{y}\| \leq \epsilon}|g(\boldsymbol{x})-g(\boldsymbol{y})| \mu_{n}(\mathrm{~d} \boldsymbol{x}, \mathrm{d} \boldsymbol{y})+\int_{\|\boldsymbol{x}-\boldsymbol{y}\|>\epsilon}|g(\boldsymbol{x})-g(\boldsymbol{y})| \mu_{n}(\mathrm{~d} \boldsymbol{x}, \mathrm{d} \boldsymbol{y}) \\
& \leq \epsilon \sup _{\boldsymbol{x}, \boldsymbol{y} \in \mathbb{R}^{k}, \boldsymbol{x} \neq \boldsymbol{y}} \frac{|g(\boldsymbol{x})-g(\boldsymbol{y})|}{\|\boldsymbol{x}-\boldsymbol{y}\|}+2 \mu_{n}\left(\left\{(\boldsymbol{x}, \boldsymbol{y}) \in \mathbb{R}^{k} \times \mathbb{R}^{k}:\|\boldsymbol{x}-\boldsymbol{y}\|>\epsilon\right\}\right) \sup _{\boldsymbol{x} \in \mathbb{R}^{k}}|g(\boldsymbol{x})|
\end{aligned}
$$

By the assumptions, for each $\epsilon \in(0, \infty)$, we get

$$
\limsup _{n \rightarrow \infty} I_{n}^{(g)} \leq \epsilon \sup _{\boldsymbol{x}, \boldsymbol{y} \in \mathbb{R}^{k}, \boldsymbol{x} \neq \boldsymbol{y}} \frac{|g(\boldsymbol{x})-g(\boldsymbol{y})|}{\|\boldsymbol{x}-\boldsymbol{y}\|},
$$

thus $\limsup _{n \rightarrow \infty} I_{n}^{(g)}=0$, and hence $\lim _{n \rightarrow \infty} I_{n}^{(g)}=0$. Consequently, for each bounded Lipschitz function $g: \mathbb{R}^{k} \rightarrow \mathbb{R}$, we obtain $\Delta_{n}^{(g)} \rightarrow 0$ as $n \rightarrow \infty$. By the portmanteau lemma (see, e.g., van der Vaart [42, Lemma 2.2]), we conclude $\mu_{n}^{(2)} \stackrel{w}{\longrightarrow} \mu^{(1)}$ as $n \rightarrow \infty$.

Lemma D.2. Let $\mu_{n}, n \in \mathbb{N}$, be probability measures on $\left(\mathbb{R}^{k+\ell}, \mathcal{B}\left(\mathbb{R}^{k+\ell}\right)\right)$ with some $k, \ell \in \mathbb{N}$. For each $n \in \mathbb{N}$, consider the marginal probability measures $\mu_{n}^{(1)}$ and $\mu_{n}^{(2)}$ on $\left(\mathbb{R}^{k}, \mathcal{B}\left(\mathbb{R}^{k}\right)\right)$ and $\left(\mathbb{R}^{\ell}, \mathcal{B}\left(\mathbb{R}^{\ell}\right)\right)$, respectively, defined by $\mu_{n}^{(1)}\left(B_{1}\right):=\mu_{n}\left(B_{1} \times \mathbb{R}^{\ell}\right)$ for $B_{1} \in \mathcal{B}\left(\mathbb{R}^{k}\right)$ and $\mu_{n}^{(2)}\left(B_{2}\right):=$ $\mu_{n}\left(\mathbb{R}^{k} \times B_{2}\right)$ for $B_{2} \in \mathcal{B}\left(\mathbb{R}^{\ell}\right)$. If $\mu_{n}^{(1)} \stackrel{w}{\longrightarrow} \mu^{(1)}$ as $n \rightarrow \infty$ with some probability measure $\mu^{(1)}$ on $\left(\mathbb{R}^{k}, \mathcal{B}\left(\mathbb{R}^{k}\right)\right.$ ) and $\mu_{n}^{(2)} \stackrel{w}{\longrightarrow} \delta_{\boldsymbol{c}}$ as $n \rightarrow \infty$ with some $\boldsymbol{c} \in \mathbb{R}^{\ell}$, then $\mu_{n} \stackrel{w}{\longrightarrow} \mu^{(1)} \times \delta_{\boldsymbol{c}}$ as $n \rightarrow \infty$.

Proof. For each $n \in \mathbb{N}$, consider the probability measure $\widetilde{\mu}_{n}$ on $\left(\mathbb{R}^{k+\ell} \times \mathbb{R}^{k+\ell}, \mathcal{B}\left(\mathbb{R}^{k+\ell} \times \mathbb{R}^{k+\ell}\right)\right)$ defined by

$$
\widetilde{\mu}_{n}(H):=\mu_{n}\left(\left\{(\boldsymbol{x}, \boldsymbol{y}) \in \mathbb{R}^{k} \times \mathbb{R}^{\ell}:(\boldsymbol{x}, \boldsymbol{y}, \boldsymbol{x}, \boldsymbol{c}) \in H\right\}\right), \quad H \in \mathcal{B}\left(\mathbb{R}^{k+\ell} \times \mathbb{R}^{k+\ell}\right) .
$$

For each $n \in \mathbb{N}$, consider the marginal probability measures $\widetilde{\mu}_{n}^{(1)}$ and $\widetilde{\mu}_{n}^{(2)}$ on $\left(\mathbb{R}^{k+\ell}, \mathcal{B}\left(\mathbb{R}^{k+\ell}\right)\right)$ defined by $\widetilde{\mu}_{n}^{(1)}(A):=\widetilde{\mu}_{n}\left(A \times \mathbb{R}^{k+\ell}\right)$ and $\widetilde{\mu}_{n}^{(2)}(A):=\widetilde{\mu}_{n}\left(\mathbb{R}^{k+\ell} \times A\right)$ for $A \in \mathcal{B}\left(\mathbb{R}^{k+\ell}\right)$. Note that for each $n \in \mathbb{N}$ and $A \in \mathcal{B}\left(\mathbb{R}^{k+\ell}\right)$, we have

$$
\widetilde{\mu}_{n}^{(1)}(A)=\mu_{n}\left(\left\{(\boldsymbol{x}, \boldsymbol{y}) \in \mathbb{R}^{k} \times \mathbb{R}^{\ell}:(\boldsymbol{x}, \boldsymbol{y}) \in A\right\}\right)=\mu_{n}(A),
$$


hence $\widetilde{\mu}_{n}^{(1)}=\mu_{n}$. Moreover, for each $n \in \mathbb{N}$ and $A \in \mathcal{B}\left(\mathbb{R}^{k+\ell}\right)$, we have

$$
\begin{aligned}
\widetilde{\mu}_{n}^{(2)}(A) & =\mu_{n}\left(\left\{(\boldsymbol{x}, \boldsymbol{y}) \in \mathbb{R}^{k} \times \mathbb{R}^{\ell}:(\boldsymbol{x}, \boldsymbol{c}) \in A\right\}\right) \\
& =\mu_{n}\left(\left\{\boldsymbol{x} \in \mathbb{R}^{k}:(\boldsymbol{x}, \boldsymbol{c}) \in A\right\} \times \mathbb{R}^{\ell}\right) \\
& =\int_{\mathbb{R}^{\ell}} \mu_{n}\left(\left\{\boldsymbol{x} \in \mathbb{R}^{k}:(\boldsymbol{x}, \boldsymbol{y}) \in A\right\} \times \mathbb{R}^{\ell}\right) \delta_{\boldsymbol{c}}(\mathrm{d} \boldsymbol{y}) \\
& =\int_{\mathbb{R}^{\ell}} \mu_{n}^{(1)}\left(\left\{\boldsymbol{x} \in \mathbb{R}^{k}:(\boldsymbol{x}, \boldsymbol{y}) \in A\right\}\right) \delta_{\boldsymbol{c}}(\mathrm{d} \boldsymbol{y}) \\
& =\int_{\mathbb{R}^{\ell}}\left(\int_{\mathbb{R}^{k}} \mathbb{1}_{A}(\boldsymbol{x}, \boldsymbol{y}) \mu_{n}^{(1)}(\mathrm{d} \boldsymbol{x})\right) \delta_{\boldsymbol{c}}(\mathrm{d} \boldsymbol{y})=\left(\mu_{n}^{(1)} \times \delta_{\boldsymbol{c}}\right)(A),
\end{aligned}
$$

hence $\widetilde{\mu}_{n}^{(2)}=\mu_{n}^{(1)} \times \delta_{\boldsymbol{c}}$. Further, for each $\epsilon \in(0, \infty)$, we have

$$
\begin{aligned}
& \widetilde{\mu}_{n}\left(\left\{((\boldsymbol{x}, \boldsymbol{y}),(\boldsymbol{u}, \boldsymbol{v})) \in \mathbb{R}^{k+\ell} \times \mathbb{R}^{k+\ell}:\|(\boldsymbol{x}, \boldsymbol{y})-(\boldsymbol{u}, \boldsymbol{v})\|>\epsilon\right\}\right) \\
& =\mu_{n}\left(\left\{(\boldsymbol{x}, \boldsymbol{y}) \in \mathbb{R}^{k} \times \mathbb{R}^{\ell}:\|(\boldsymbol{x}, \boldsymbol{y})-(\boldsymbol{x}, \boldsymbol{c})\|>\epsilon\right\}\right) \\
& =\mu_{n}\left(\left\{(\boldsymbol{x}, \boldsymbol{y}) \in \mathbb{R}^{k} \times \mathbb{R}^{\ell}:\|\boldsymbol{y}-\boldsymbol{c}\|>\epsilon\right\}\right) \\
& =\mu_{n}^{(2)}\left(\left\{\boldsymbol{y} \in \mathbb{R}^{\ell}:\|\boldsymbol{y}-\boldsymbol{c}\|>\epsilon\right\}\right) \rightarrow 0 \quad \text { as } n \rightarrow \infty,
\end{aligned}
$$

since $\mu_{n}^{(2)} \stackrel{w}{\longrightarrow} \delta_{c}$ as $n \rightarrow \infty$. Thus, according to Lemma D.1, to prove the statement it suffices to show that $\mu_{n}^{(1)} \times \delta_{c} \stackrel{w}{\longrightarrow} \mu^{(1)} \times \delta_{c}$ as $n \rightarrow \infty$. For every continuous, bounded function $g: \mathbb{R}^{k+\ell} \times \mathbb{R}^{k+\ell} \rightarrow \mathbb{R}$, by the portmanteau lemma (see, e.g., van der Vaart [42, Lemma 2.2]), we have

$$
\begin{aligned}
& \int_{\mathbb{R}^{k+\ell}} g(\boldsymbol{x}, \boldsymbol{z})\left(\mu_{n}^{(1)} \times \delta_{\boldsymbol{c}}\right)(\mathrm{d} \boldsymbol{x}, \mathrm{d} \boldsymbol{z})=\int_{\mathbb{R}^{k}} g(\boldsymbol{x}, \boldsymbol{c}) \mu_{n}^{(1)}(\mathrm{d} \boldsymbol{x}) \\
& \rightarrow \int_{\mathbb{R}^{k}} g(\boldsymbol{x}, \boldsymbol{c}) \mu^{(1)}(\mathrm{d} \boldsymbol{x})=\int_{\mathbb{R}^{k+\ell}} g(\boldsymbol{x}, \boldsymbol{z})\left(\mu^{(1)} \times \delta_{\boldsymbol{c}}\right)(\mathrm{d} \boldsymbol{x}, \mathrm{d} \boldsymbol{z})
\end{aligned}
$$

as $n \rightarrow \infty$, since $\mu_{n}^{(1)} \stackrel{w}{\longrightarrow} \mu^{(1)}$ as $n \rightarrow \infty$, and the function $\mathbb{R}^{k} \ni \boldsymbol{x} \mapsto g(\boldsymbol{x}, \boldsymbol{c}) \in \mathbb{R}$ is a continuous, bounded function. Again by the portmanteau lemma, we conclude $\mu_{n}^{(1)} \times \delta_{\boldsymbol{c}} \stackrel{w}{\longrightarrow}$ $\mu^{(1)} \times \delta_{c}$ as $n \rightarrow \infty$, as desired.

We will use the following conditional continuous mapping theorem and a consequence of it. Recall that for a random vector $\boldsymbol{X}$ and an event $A \in \mathcal{A}$ such that $\mathbb{P}(A)>0$, the conditional law of $\boldsymbol{X}$ with respect to $A$ is denoted by $\mathcal{L}(\boldsymbol{X} \mid A)$.

Lemma D.3. For each $n \in \mathbb{N}$, let $A_{n} \in \mathcal{A}$ such that $\mathbb{P}\left(A_{n}\right)>0$. Let $\boldsymbol{X}$ and $\boldsymbol{X}_{n}, n \in \mathbb{N}$, be $\mathbb{R}^{k}$-valued random vectors and let $h: \mathbb{R}^{k} \rightarrow \mathbb{R}^{\ell}$ be a Borel measurable function with some $k, \ell \in \mathbb{N}$. Suppose that $\mathcal{L}\left(\boldsymbol{X}_{n} \mid A_{n}\right) \stackrel{w}{\longrightarrow} \mathcal{L}(\boldsymbol{X})$ as $n \rightarrow \infty$ and $\mathbb{P}\left(\boldsymbol{X} \in D_{h}\right)=0$, where $D_{h}$ denotes the set of discontinuities of $h$.

(i) Then $\mathcal{L}\left(h\left(\boldsymbol{X}_{n}\right) \mid A_{n}\right) \stackrel{w}{\longrightarrow} \mathcal{L}(h(\boldsymbol{X}))$ as $n \rightarrow \infty$. 
(ii) If, in addition, $h$ is bounded, then $\mathbb{E}\left[h\left(\boldsymbol{X}_{n}\right) \mid A_{n}\right] \rightarrow \mathbb{E}[h(\boldsymbol{X})]$ as $n \rightarrow \infty$.

Proof. (i). For each $B \in \mathcal{B}\left(\mathbb{R}^{k}\right)$, let $\mu(B):=\mathbb{P}(\boldsymbol{X} \in B)$ and $\mu_{n}(B):=\mathbb{P}\left(\boldsymbol{X}_{n} \in B \mid A_{n}\right)$, $n \in \mathbb{N}$. By the assumption, $\mu_{n} \stackrel{w}{\longrightarrow} \mu$ as $n \rightarrow \infty$. By Billingsley [8, Lemma 5.1], we obtain $\nu_{n} \stackrel{w}{\longrightarrow} \nu$ as $n \rightarrow \infty$, where the probability measures $\nu_{n}, n \in \mathbb{N}$, and $\nu$ on $\left(\mathbb{R}^{\ell}, \mathcal{B}\left(\mathbb{R}^{\ell}\right)\right)$ are defined by $\nu_{n}(B):=\mu_{n}\left(h^{-1}(B)\right)=\mathbb{P}\left(\boldsymbol{X}_{n} \in h^{-1}(B) \mid A_{n}\right)=\mathbb{P}\left(h\left(\boldsymbol{X}_{n}\right) \in B \mid A_{n}\right), n \in \mathbb{N}$, and $\nu(B):=\mu\left(h^{-1}(B)\right)=\mathbb{P}\left(\boldsymbol{X} \in h^{-1}(B)\right)=\mathbb{P}(h(\boldsymbol{X}) \in B)$ for $B \in \mathcal{B}\left(\mathbb{R}^{\ell}\right)$. Consequently, we obtain $\mathcal{L}\left(h\left(\boldsymbol{X}_{n}\right) \mid A_{n}\right) \stackrel{w}{\longrightarrow} \mathcal{L}(h(\boldsymbol{X}))$ as $n \rightarrow \infty$, as desired.

(ii). By Billingsley [8, part (iii) of Lemma 5.2], we obtain $\int_{\mathbb{R}^{k}} h(\boldsymbol{x}) \mu_{n}(\mathrm{~d} \boldsymbol{x}) \rightarrow \int_{\mathbb{R}^{k}} h(\boldsymbol{x}) \mu(\mathrm{d} \boldsymbol{x})$ as $n \rightarrow \infty$. Consequently, we obtain $\mathbb{E}\left[h\left(\boldsymbol{X}_{n}\right) \mid A_{n}\right] \rightarrow \mathbb{E}[h(\boldsymbol{X})]$ as $n \rightarrow \infty$, as desired.

Next, we prove a conditional analogue of part (v) of Theorem 2.7 in van der Vaart [42] together with one of its useful consequences.

Lemma D.4. For each $n \in \mathbb{N}$, let $A_{n} \in \mathcal{A}$ such that $\mathbb{P}\left(A_{n}\right)>0$. Let $\boldsymbol{X}$ and $\boldsymbol{X}_{n}, n \in \mathbb{N}$, be $\mathbb{R}^{k}$-valued random vectors and let $\boldsymbol{Y}_{n}, n \in \mathbb{N}$, be $\mathbb{R}^{\ell}$-valued random vectors with some $k, \ell \in \mathbb{N}$. Suppose that $\mathcal{L}\left(\boldsymbol{X}_{n} \mid A_{n}\right) \stackrel{w}{\longrightarrow} \mathcal{L}(\boldsymbol{X})$ and $\mathcal{L}\left(\boldsymbol{Y}_{n} \mid A_{n}\right) \stackrel{w}{\longrightarrow} \delta_{\boldsymbol{c}}$ as $n \rightarrow \infty$ with some $\boldsymbol{c} \in \mathbb{R}^{\ell}$.

(i) Then $\mathcal{L}\left(\left(\boldsymbol{X}_{n}, \boldsymbol{Y}_{n}\right) \mid A_{n}\right) \stackrel{w}{\longrightarrow} \mathcal{L}(\boldsymbol{X}) \times \delta_{\boldsymbol{c}}=\mathcal{L}((\boldsymbol{X}, \boldsymbol{c}))$ as $n \rightarrow \infty$.

(ii) If, in addition, $h: \mathbb{R}^{k+\ell} \rightarrow \mathbb{R}^{m}$ is a Borel measurable function with some $m \in \mathbb{N}$ such that $h$ is continuous at every $\left(\boldsymbol{x}_{0}, \boldsymbol{c}\right), \boldsymbol{x}_{0} \in \mathbb{R}^{k}$, then $\mathcal{L}\left(h\left(\boldsymbol{X}_{n}, \boldsymbol{Y}_{n}\right) \mid A_{n}\right) \stackrel{w}{\longrightarrow} \mathcal{L}(h(\boldsymbol{X}, \boldsymbol{c}))$ as $n \rightarrow \infty$.

Proof. (i). We apply Lemma D.2 for the probability measures $\mu_{n}:=\mathcal{L}\left(\left(\boldsymbol{X}_{n}, \boldsymbol{Y}_{n}\right) \mid A_{n}\right), n \in \mathbb{N}$, on $\left(\mathbb{R}^{k+\ell}, \mathcal{B}\left(\mathbb{R}^{k+\ell}\right)\right)$. Then we have $\mu_{n}^{(1)}=\mathcal{L}\left(\boldsymbol{X}_{n} \mid A_{n}\right) \stackrel{w}{\longrightarrow} \mathcal{L}(\boldsymbol{X})$ and $\mu_{n}^{(2)}=\mathcal{L}\left(\boldsymbol{Y}_{n} \mid A_{n}\right) \stackrel{w}{\longrightarrow} \delta_{\boldsymbol{c}}$ as $n \rightarrow \infty$, hence we obtain $\mathcal{L}\left(\left(\boldsymbol{X}_{n}, \boldsymbol{Y}_{n}\right) \mid A_{n}\right)=\mu_{n} \stackrel{w}{\longrightarrow} \mathcal{L}(\boldsymbol{X}) \times \delta_{\boldsymbol{c}}=\mathcal{L}((\boldsymbol{X}, \boldsymbol{c}))$ as $n \rightarrow \infty$.

(ii). By the assumption, $D_{h} \subset \mathbb{R}^{k} \times\left(\mathbb{R}^{\ell} \backslash\{\boldsymbol{c}\}\right)$, hence $\mathbb{P}\left((\boldsymbol{X}, \boldsymbol{c}) \in D_{h}\right)=0$. Consequently, part (i) of Lemma D.3 implies $\mathcal{L}\left(h\left(\boldsymbol{X}_{n}, \boldsymbol{Y}_{n}\right) \mid A_{n}\right) \stackrel{w}{\longrightarrow} \mathcal{L}(h(\boldsymbol{X}, \boldsymbol{c}))$ as $n \rightarrow \infty$.

Finally, we provide a conditional Slutsky's lemma.

Lemma D.5. For each $n \in \mathbb{N}$, let $A_{n} \in \mathcal{A}$ such that $\mathbb{P}\left(A_{n}\right)>0$. Let $\boldsymbol{X}$ and $\boldsymbol{X}_{n}, n \in \mathbb{N}$, be $\mathbb{R}^{k \times \ell}$-valued random matrices such that $\mathcal{L}\left(\boldsymbol{X}_{n} \mid A_{n}\right) \stackrel{w}{\longrightarrow} \mathcal{L}(\boldsymbol{X})$ as $n \rightarrow \infty$ with some $k, \ell \in \mathbb{N}$.

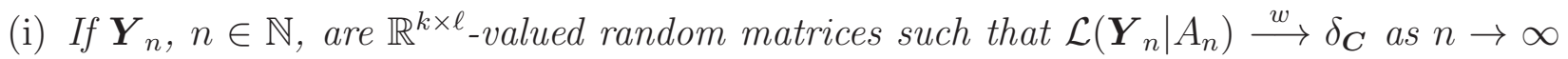
with some $\boldsymbol{C} \in \mathbb{R}^{k \times \ell}$, then $\mathcal{L}\left(\boldsymbol{X}_{n}+\boldsymbol{Y}_{n} \mid A_{n}\right) \stackrel{w}{\longrightarrow} \mathcal{L}(\boldsymbol{X}+\boldsymbol{C})$ as $n \rightarrow \infty$.

(ii) If $\boldsymbol{Y}_{n}, n \in \mathbb{N}$, are $\mathbb{R}^{m \times k}$-valued random matrices such that $\mathcal{L}\left(\boldsymbol{Y}_{n} \mid A_{n}\right) \stackrel{w}{\longrightarrow} \delta_{\boldsymbol{C}}$ as $n \rightarrow \infty$ with some $\boldsymbol{C} \in \mathbb{R}^{m \times k}$ and $m \in \mathbb{N}$, then $\mathcal{L}\left(\boldsymbol{Y}_{n} \boldsymbol{X}_{n} \mid A_{n}\right) \stackrel{w}{\longrightarrow} \mathcal{L}(\boldsymbol{C X})$ as $n \rightarrow \infty$.

(iii) If $\boldsymbol{Y}_{n}, n \in \mathbb{N}$, are $\mathbb{R}^{k \times k}$-valued random matrices such that $\mathcal{L}\left(\boldsymbol{Y}_{n} \mid A_{n}\right) \stackrel{w}{\longrightarrow} \delta_{\boldsymbol{C}}$ as $n \rightarrow \infty$ with some invertible $\boldsymbol{C} \in \mathbb{R}^{k \times k}$, then $\mathcal{L}\left(\boldsymbol{Y}_{n}^{-1} \boldsymbol{X}_{n} \mid A_{n}\right) \stackrel{w}{\longrightarrow} \mathcal{L}\left(\boldsymbol{C}^{-1} \boldsymbol{X}\right)$ as $n \rightarrow \infty$. 
Proof. Identifying $\mathbb{R}^{k \times \ell}, \mathbb{R}^{m \times k}$ and $\mathbb{R}^{m \times \ell}$ with $\mathbb{R}^{k \ell}, \mathbb{R}^{m k}$ and $\mathbb{R}^{m \ell}$, respectively, in a natural way, we can apply part (ii) of Lemma D.4 for the Borel measurable functions

$$
\begin{gathered}
\mathbb{R}^{k \times \ell} \times \mathbb{R}^{k \times \ell} \ni(\boldsymbol{U}, \boldsymbol{V}) \mapsto \boldsymbol{U}+\boldsymbol{V} \in \mathbb{R}^{k \times \ell}, \quad \mathbb{R}^{k \times \ell} \times \mathbb{R}^{m \times k} \ni(\boldsymbol{U}, \boldsymbol{V}) \mapsto \boldsymbol{V} \in \mathbb{R}^{m \times \ell}, \\
\mathbb{R}^{k \times \ell} \times \mathbb{R}^{k \times k} \ni(\boldsymbol{U}, \boldsymbol{V}) \mapsto \begin{cases}\boldsymbol{V}^{-1} \boldsymbol{U} \in \mathbb{R}^{k \times \ell}, & \text { if } \boldsymbol{U} \text { is invertible, } \\
\mathbf{0} \in \mathbb{R}^{k \times \ell}, & \text { otherwise, }\end{cases}
\end{gathered}
$$

and we obtain the statements.

\section{E Regular variation of a related process}

First, we recall Karamata's theorem for truncated moments, see, e.g., Bingham et al. [9, pages 26-27] or Buraczewski et al. [10, Appendix B.4].

Lemma E.1 (Karamata's theorem for truncated moments). Consider a non-negative regularly varying random variable $X$ with tail index $\alpha>0$. Then

$$
\begin{aligned}
& \lim _{x \rightarrow \infty} \frac{x^{\beta} \mathbb{P}(X>x)}{\mathbb{E}\left(X^{\beta} \mathbb{1}_{\{X \leq x\}}\right)}=\frac{\beta-\alpha}{\alpha}, \quad \text { if } \beta \in[\alpha, \infty), \\
& \lim _{x \rightarrow \infty} \frac{x^{\beta} \mathbb{P}(X>x)}{\mathbb{E}\left(X^{\beta} \mathbb{1}_{\{X>x\}}\right)}=\frac{\alpha-\beta}{\alpha}, \quad \text { if } \beta \in(-\infty, \alpha) .
\end{aligned}
$$

Now, we give a representation of the strongly stationary Markov chain $\left(X_{i}\right)_{i \in \mathbb{Z}}$.

Lemma E.2. We have

$$
\left(X_{k}\right)_{k \in \mathbb{Z}} \stackrel{d}{=}\left(B_{k}+\sum_{i=1}^{\infty} \theta_{k}^{(k-i)} \circ \cdots \circ \theta_{k-i+1}^{(k-i)} \circ B_{k-i}\right)_{k \in \mathbb{Z}}
$$

where $\left\{B_{k}: k \in \mathbb{Z}\right\}$ are independent random variables with the same distribution as $B$, and $\theta_{k}^{(\ell)}, k, \ell \in \mathbb{Z}$, are given by

$$
\theta_{k}^{(\ell)} \circ i:= \begin{cases}\sum_{j=1}^{i} A_{k, j}^{(\ell)}, & \text { if } i \in \mathbb{N} \\ 0, & \text { if } i=0\end{cases}
$$

where $A_{k, j}^{(\ell)}, j \in \mathbb{N}, k, \ell \in \mathbb{Z}$, have the same distribution as $A$, and $\left\{B_{k}: k \in \mathbb{Z}\right\}$ and $\theta_{k}^{(\ell)}$, $k, \ell \in \mathbb{Z}$, are independent in the sense that the families $\left\{B_{k}: k \in \mathbb{Z}\right\}$ and $\left\{A_{k, j}^{(\ell)}: j \in \mathbb{N}\right\}$, $k, \ell \in \mathbb{Z}$, occurring in $\theta_{k}^{(\ell)}, k, \ell \in \mathbb{Z}$, are independent families of independent random variables, and the series in the representation (E.1) converge with probability one. 
Proof. Due to Basrak et al. [5, Lemma 2.2.1], the series in the representation (E.1) converge with probability one. Clearly, for each $k \in \mathbb{Z}$ and $\ell \in \mathbb{N}$, we have

$$
\begin{aligned}
& \left(B_{k}+\sum_{i=1}^{\infty} \theta_{k}^{(k-i)} \circ \cdots \circ \theta_{k-i+1}^{(k-i)} \circ B_{k-i}, \ldots, B_{k+\ell}+\sum_{i=1}^{\infty} \theta_{k+\ell}^{(k+\ell-i)} \circ \cdots \circ \theta_{k+\ell-i+1}^{(k+\ell-i)} \circ B_{k+\ell-i}\right) \\
& \stackrel{d}{=}\left(B_{0}+\sum_{i=1}^{\infty} \theta_{0}^{(-i)} \circ \cdots \circ \theta_{-i+1}^{(-i)} \circ B_{-i}, \ldots, B_{\ell}+\sum_{i=1}^{\infty} \theta_{\ell}^{(\ell-i)} \circ \cdots \circ \theta_{\ell-i+1}^{(\ell-i)} \circ B_{\ell-i}\right) .
\end{aligned}
$$

Indeed, since $\left\{B_{i}: i \in \mathbb{Z}\right\}$ are identically distributed, independent of $\left\{\theta_{i}^{(j)}: i, j \in \mathbb{Z}\right\}$, and for each $N \in \mathbb{N}$ and $i_{1}, \ldots, i_{N} \geq 0$, the distribution of the random vector $\left(\theta_{k}^{(k-1)} \circ i_{1}, \ldots, \theta_{k}^{(k-N)} \circ\right.$ $\left.\cdots \circ \theta_{k-N+1}^{(k-N)} \circ i_{N}\right)$ is invariant with respect to a shift of $k \in \mathbb{Z}$, we have

$$
\begin{aligned}
& \left(B_{k}+\sum_{i=1}^{n} \theta_{k}^{(k-i)} \circ \cdots \circ \theta_{k-i+1}^{(k-i)} \circ B_{k-i}, \ldots, B_{k+\ell}+\sum_{i=1}^{n} \theta_{k+\ell}^{(k+\ell-i)} \circ \cdots \circ \theta_{k+\ell-i+1}^{(k+\ell-i)} \circ B_{k+\ell-i}\right)_{n \in \mathbb{N}} \\
& \stackrel{d}{=}\left(B_{0}+\sum_{i=1}^{n} \theta_{0}^{(-i)} \circ \cdots \circ \theta_{-i+1}^{(-i)} \circ B_{-i}, \ldots, B_{\ell}+\sum_{i=1}^{n} \theta_{\ell}^{(\ell-i)} \circ \cdots \circ \theta_{\ell-i+1}^{(\ell-i)} \circ B_{\ell-i}\right)_{n \in \mathbb{N}} .
\end{aligned}
$$

Thus using that for a sequence of random variables $\xi_{i}, i \in \mathbb{N}$, the series $\sum_{i=1}^{\infty} \xi_{i}$ is convergent almost surely if and only if $\mathbb{P}\left(\sup _{m \in \mathbb{N}}\left|\sum_{i=n}^{n+m} \xi_{i}\right|>\epsilon\right) \rightarrow 0$ as $n \rightarrow \infty$ for each $\epsilon>0$, the almost sure convergence of the series on the left and right hand sides of (E.2) yields (E.2) (for a similar argument, see Step 2 of the proof of Theorem 4.1). Hence the right hand side of (E.1) defines a strongly stationary process. Moreover, for each $k \in \mathbb{Z}$, we have

$$
\begin{aligned}
& B_{k}+\sum_{i=1}^{\infty} \theta_{k}^{(k-i)} \circ \cdots \circ \theta_{k-i+1}^{(k-i)} \circ B_{k-i} \\
& =B_{k}+\theta_{k}^{(k-1)} \circ B_{k-1}+\theta_{k}^{(k-2)} \circ \theta_{k-1}^{(k-2)} \circ B_{k-2}+\theta_{k}^{(k-3)} \circ \theta_{k-1}^{(k-3)} \circ \theta_{k-2}^{(k-3)} \circ B_{k-2}+\cdots \\
& \stackrel{d}{=} B_{k}+\theta_{k}^{(k-1)} \circ\left(B_{k-1}+\theta_{k-1}^{(k-2)} \circ B_{k-2}+\theta_{k-1}^{(k-3)} \circ \theta_{k-2}^{(k-3)} \circ B_{k-3}+\cdots\right) \\
& =B_{k}+\theta_{k}^{(k-1)} \circ\left(B_{k-1}+\sum_{i=1}^{\infty} \theta_{k-1}^{(k-i-1)} \circ \cdots \circ \theta_{k-i}^{(k-i-1)} \circ B_{k-1-i}\right),
\end{aligned}
$$

since $\theta_{k}^{(k-i)}, i \in \mathbb{N}$, are independent, and independent of $\left\{B_{k-i}: i \geq 0\right\} \cup\left\{\theta_{k-\ell}^{(k-i-1)}: i, \ell \in \mathbb{N}\right\}$. Consequently, the stochastic process given on the right hand side of (E.1) is a time homogeneous Markov process with the same transition probabilities as the Galton-Watson process $\left(X_{k}\right)_{k \in \mathbb{Z}}$ with immigration satisfying (1.3) such that the distribution of $X_{0}$ is the unique stationary distribution of $\left(X_{k}\right)_{k \in \mathbb{Z}}$.

It turns out that the process $\left(X_{i}^{3 / 2}, X_{i} M_{i+1}\right)_{i \in \mathbb{Z}}$ is regularly varying with tail index $\frac{2 \alpha}{3}$ with an explicitly given forward tail process.

Theorem E.3. As $x \rightarrow \infty$,

$$
\mathcal{L}\left(\left(\frac{1}{x}\left(X_{k}^{3 / 2}, X_{k} M_{k+1}\right)\right)_{k \geq 0} \mid\left(X_{0}^{3 / 2} \vee X_{0}\left|M_{1}\right|\right)>x\right) \stackrel{\text { fi.di. }}{\longrightarrow} \mathcal{L}\left(\left(\mu_{A}^{3 k / 2} \widetilde{Y}, \mu_{A}^{3 k / 2} \widetilde{Y} \widetilde{Z}_{k}\right)_{k \geq 0}\right),
$$


where $\widetilde{Z}_{k}, k \in \mathbb{N}$, is an i.i.d. sequence of $\mathcal{N}\left(0, \sigma_{A}^{2}\right)$-distributed random variables, the distribution of $\left(\widetilde{Y}, \widetilde{Z}_{0}\right)$ is given by

$$
\mathbb{P}\left(\widetilde{Y}>y, \widetilde{Z}_{0}>v_{0}\right)=\frac{\mathbb{E}\left(\left(y \vee\left(1 \vee\left|Z_{0}\right|\right)^{-1}\right)^{-2 \alpha / 3} \mathbb{1}_{\left(v_{0}, \infty\right)}\left(Z_{0}\right)\right)}{\mathbb{E}\left(\left(1 \vee\left|Z_{0}\right|\right)^{2 \alpha / 3}\right)}
$$

for $y, v_{0} \in \mathbb{R}$, where $Z_{0} \stackrel{d}{=} \mathcal{N}\left(0, \sigma_{A}^{2}\right)$, and the random vector $\left(\widetilde{Y}, \widetilde{Z}_{0}\right)$ is independent from the variables $\widetilde{Z}_{k}, k \in \mathbb{N}$. Consequently, the strongly stationary process $\left(X_{k}^{3 / 2}, X_{k} M_{k+1}\right)_{k \in \mathbb{Z}}$ is jointly regularly varying with tail index $\frac{2 \alpha}{3}$, i.e., all its finite dimensional distributions are regularly varying with tail index $\frac{2 \alpha}{3}$. The process

$$
\left(\mu_{A}^{3 k / 2} \widetilde{Y}, \mu_{A}^{3 k / 2} \widetilde{Y} \widetilde{Z}_{k}\right)_{k \geq 0}
$$

is the forward tail process of $\left(X_{k}^{3 / 2}, X_{k} M_{k+1}\right)_{k \in \mathbb{Z}}$. Moreover, there exists a (whole) tail process of $\left(X_{k}^{3 / 2}, X_{k} M_{k+1}\right)_{k \in \mathbb{Z}}$ as well.

Proof. By Lemma E.2, we may and do suppose that $\left(X_{k}\right)_{k \geq 0}$ is the right hand side of (E.1). First, we give a useful representation of the random vectors $\left(X_{0}, X_{1}, \ldots, X_{n}, M_{1}, \ldots, M_{n+1}\right)$, $n \in \mathbb{N}$. For each $k \in \mathbb{N}$, we obtain

$$
\begin{aligned}
X_{k}= & B_{k}+\sum_{i=1}^{\infty} \theta_{k}^{(k-i)} \circ \cdots \circ \theta_{k-i+1}^{(k-i)} \circ B_{k-i} \\
= & B_{k}+\sum_{i=1}^{k-1} \theta_{k}^{(k-i)} \circ \cdots \circ \theta_{k-i+1}^{(k-i)} \circ B_{k-i} \\
& +\theta_{k}^{(0)} \circ \cdots \circ \theta_{1}^{(0)} \circ B_{0}+\sum_{i=k+1}^{\infty} \theta_{k}^{(k-i)} \circ \cdots \circ \theta_{k-i+1}^{(k-i)} \circ B_{k-i} \\
& \stackrel{d}{=} \kappa_{k}+\theta_{k}^{(0)} \circ \cdots \circ \theta_{1}^{(0)} \circ\left(B_{0}+\sum_{j=1}^{\infty} \theta_{0}^{(-j)} \circ \cdots \circ \theta_{-j+1}^{(-j)} \circ B_{-j}\right) \\
= & \kappa_{k}+\theta_{k}^{(0)} \circ \cdots \circ \theta_{1}^{(0)} \circ X_{0},
\end{aligned}
$$

where $\kappa_{k}:=B_{k}+\sum_{i=1}^{k-1} \theta_{k}^{(k-i)} \circ \cdots \circ \theta_{k-i+1}^{(k-i)} \circ B_{k-i}$, since for each $k \in \mathbb{N}, \theta_{k}^{(0)} \circ \cdots \circ \theta_{1}^{(0)} \circ \theta_{0}^{(-j)} \circ$ $\cdots \circ \theta_{-j+1}^{(-j)} \circ B_{-j}, j \geq 1$, has the same distribution as $\theta_{k}^{(k-i)} \circ \cdots \circ \theta_{k-i+1}^{(k-i)} \circ B_{k-i}, i \geq k+1$, and $\theta_{k}^{(0)} \circ \cdots \circ \theta_{1}^{(0)} \circ B_{0}, \theta_{k}^{(0)} \circ \cdots \circ \theta_{1}^{(0)} \circ \theta_{0}^{(-j)} \circ \cdots \circ \theta_{-j+1}^{(-j)} \circ B_{-j}, j \geq 1$, and $\kappa_{k}$ are independent. Note also that $\kappa_{k}, \theta_{k}^{(0)} \circ \cdots \circ \theta_{1}^{(0)}$ and $X_{0}$ are independent for any $k \in \mathbb{N}$ (in the sense given in Lemma E.2). In the same way, we get

$$
\left(X_{0}, X_{1}, \ldots, X_{k}\right) \stackrel{d}{=}\left(X_{0}, \kappa_{1}+\theta_{1}^{(0)} \circ X_{0}, \ldots, \kappa_{k}+\theta_{k}^{(0)} \circ \cdots \circ \theta_{1}^{(0)} \circ X_{0}\right) .
$$


Moreover, for each $k \in \mathbb{N}$, using (E.4) for $\left(X_{0}, X_{1}, \ldots, X_{k+1}\right)$, we obtain

$$
\begin{aligned}
& M_{k+1}=X_{k+1}-\mu_{A} X_{k}-\mu_{B} \\
\stackrel{d}{=} & \kappa_{k+1}+\theta_{k+1}^{(0)} \circ \cdots \circ \theta_{1}^{(0)} \circ X_{0}-\mu_{A}\left(\kappa_{k}+\theta_{k}^{(0)} \circ \cdots \circ \theta_{1}^{(0)} \circ X_{0}\right)-\mu_{B} \\
= & B_{k+1}+\sum_{i=1}^{k} \theta_{k+1}^{(k+1-i)} \circ \cdots \circ \theta_{k+2-i}^{(k+1-i)} \circ B_{k+1-i}+\theta_{k+1}^{(0)} \circ \cdots \circ \theta_{1}^{(0)} \circ X_{0} \\
& -\mu_{A}\left(B_{k}+\sum_{i=1}^{k-1} \theta_{k}^{(k-i)} \circ \cdots \circ \theta_{k+1-i}^{(k-i)} \circ B_{k-i}\right)-\mu_{A}\left(\theta_{k}^{(0)} \circ \cdots \circ \theta_{1}^{(0)} \circ X_{0}\right)-\mu_{B} \\
= & B_{k+1}-\mu_{B}+\theta_{k+1}^{(k)} \circ B_{k}-\mu_{A} B_{k} \\
& +\sum_{i=2}^{k} \theta_{k+1}^{(k+1-i)} \circ \cdots \circ \theta_{k+2-i}^{(k+1-i)} \circ B_{k+1-i}-\mu_{A} \sum_{i=1}^{k-1} \theta_{k}^{(k-i)} \circ \cdots \circ \theta_{k+1-i}^{(k-i)} \circ B_{k-i} \\
& +\theta_{k+1}^{(0)} \circ \cdots \circ \theta_{1}^{(0)} \circ X_{0}-\mu_{A}\left(\theta_{k}^{(0)} \circ \cdots \circ \theta_{1}^{(0)} \circ X_{0}\right) \\
= & B_{k+1}-\mu_{B}+\sum_{i=0}^{k-1} \widetilde{\theta}_{k+1}^{(k-i)} \circ \theta_{k}^{(k-i)} \circ \cdots \circ \theta_{k+1-i}^{(k-i)} \circ B_{k-i}+\widetilde{\theta}_{k+1}^{(0)} \circ \theta_{k}^{(0)} \circ \cdots \circ \theta_{1}^{(0)} \circ X_{0} \\
= & \widetilde{\kappa}_{k+1}+\widetilde{\theta}_{k+1}^{(0)} \circ \theta_{k}^{(0)} \circ \cdots \circ \theta_{1}^{(0)} \circ X_{0},
\end{aligned}
$$

where $\widetilde{\theta}_{k}^{(\ell)}, k, \ell \in \mathbb{Z}$, are given by

$$
\widetilde{\theta}_{k}^{(\ell)} \circ i:= \begin{cases}\theta_{k}^{(\ell)} \circ i-i \mu_{A}=\sum_{j=1}^{i}\left(A_{k, j}^{(\ell)}-\mu_{A}\right), & \text { for } i \in \mathbb{N}, \\ 0, & \text { for } i=0,\end{cases}
$$

and $\widetilde{\kappa}_{k+1}:=B_{k+1}-\mu_{B}+\sum_{i=0}^{k-1} \widetilde{\theta}_{k+1}^{(k-i)} \circ \theta_{k}^{(k-i)} \circ \cdots \circ \theta_{k+1-i}^{(k-i)} \circ B_{k-i}$. Note that

$$
\begin{aligned}
\widetilde{\kappa}_{k+1} & =B_{k+1}-\mu_{B}+\widetilde{\theta}_{k+1}^{(k)} \circ B_{k}+\sum_{i=1}^{k-1} \widetilde{\theta}_{k+1}^{(k-i)} \circ \theta_{k}^{(k-i)} \circ \cdots \circ \theta_{k+1-i}^{(k-i)} \circ B_{k-i} \\
& \stackrel{d}{=} B_{k+1}-\mu_{B}+\widetilde{\theta}_{k+1}^{(k)} \circ \kappa_{k},
\end{aligned}
$$

since $\widetilde{\theta}_{k+1}^{(k-i)} \circ j, i \in\{0, \ldots, k-1\}, j \geq 0$, are independent having the same distribution as $\widetilde{\theta}_{k+1}^{(k)} \circ j, j \geq 0$, and $\widetilde{\theta}_{k+1}^{(k)}$ is independent of $B_{k+1}$. Further, $\widetilde{\kappa}_{k+1}, \widetilde{\theta}_{k+1}^{(0)} \circ \theta_{k}^{(0)} \circ \cdots \circ \theta_{1}^{(0)}$ and $X_{0}$ are independent (in the sense given in Lemma E.2 ). Moreover, we have $M_{1}=\widetilde{\theta}_{1}^{(0)} \circ X_{0}+B_{1}-\mu_{B}=$ $\widetilde{\kappa}_{1}+\widetilde{\theta}_{1}^{(0)} \circ X_{0}$ with $\widetilde{\kappa}_{1}:=B_{1}-\mu_{B}$. In the same way, we get

$$
\begin{aligned}
& \left(X_{0}, X_{1}, \ldots, X_{n}, M_{1}, \ldots, M_{n+1}\right) \\
& \stackrel{d}{=}\left(X_{0}, \kappa_{1}+\theta_{1}^{(0)} \circ X_{0}, \ldots, \kappa_{n}+\theta_{n}^{(0)} \circ \cdots \circ \theta_{1}^{(0)} \circ X_{0},\right. \\
& \left.\quad \widetilde{\kappa}_{1}+\widetilde{\theta}_{1}^{(0)} \circ X_{0}, \ldots, \widetilde{\kappa}_{n+1}+\widetilde{\theta}_{n+1}^{(0)} \circ \theta_{n}^{(0)} \circ \cdots \circ \theta_{1}^{(0)} \circ X_{0}\right), \quad n \geq 0 .
\end{aligned}
$$


Step 1. First, we check that

$$
\mathcal{L}\left(\frac{X_{0}^{3 / 2}}{x} \mid\left(X_{0}^{3 / 2} \vee X_{0}\left|M_{1}\right|\right)>x\right) \stackrel{w}{\longrightarrow} \mathcal{L}(\tilde{Y}) \quad \text { as } \quad x \rightarrow \infty .
$$

For each $x, y \in(0, \infty)$, we have

$$
\mathbb{P}\left(\frac{X_{0}^{3 / 2}}{x}>y \mid\left(X_{0}^{3 / 2} \vee X_{0}\left|M_{1}\right|\right)>x\right)=\frac{P_{1}(x, y)}{Q_{1}(x)}
$$

with

$$
P_{1}(x, y):=\frac{\mathbb{P}\left(X_{0}^{3 / 2}>x y,\left(X_{0}^{3 / 2} \vee X_{0}\left|M_{1}\right|\right)>x\right)}{\mathbb{P}\left(X_{0}^{3 / 2}>x\right)}, \quad Q_{1}(x):=\frac{\mathbb{P}\left(\left(X_{0}^{3 / 2} \vee X_{0}\left|M_{1}\right|\right)>x\right)}{\mathbb{P}\left(X_{0}^{3 / 2}>x\right)} .
$$

For each $x \in(0, \infty)$ and $c \in(0,1)$, we can write $Q_{1}(x)=Q_{1,1}(x, c)+Q_{1,2}(x, c)$ with

$$
\begin{aligned}
Q_{1,1}(x, c) & :=\frac{\mathbb{P}\left(\left(X_{0}^{3 / 2} \vee X_{0}\left|M_{1}\right|\right)>x, X_{0}^{3 / 2}>c x\right)}{\mathbb{P}\left(X_{0}^{3 / 2}>x\right)}, \\
Q_{1,2}(x, c) & :=\frac{\mathbb{P}\left(\left(X_{0}^{3 / 2} \vee X_{0}\left|M_{1}\right|\right)>x, X_{0}^{3 / 2} \leq c x\right)}{\mathbb{P}\left(X_{0}^{3 / 2}>x\right)},
\end{aligned}
$$

where $Q_{1,1}(x, c)=Q_{1,1,1}(x, c) Q_{1,1,2}(x, c)$ with

$$
Q_{1,1,1}(x, c):=\frac{\mathbb{P}\left(\left(X_{0}^{3 / 2} \vee X_{0}\left|M_{1}\right|\right)>x, X_{0}^{3 / 2}>c x\right)}{\mathbb{P}\left(X_{0}^{3 / 2}>c x\right)}, \quad Q_{1,1,2}(x, c):=\frac{\mathbb{P}\left(X_{0}^{3 / 2}>c x\right)}{\mathbb{P}\left(X_{0}^{3 / 2}>x\right)} .
$$

For each $c \in(0,1)$, we have

$$
\lim _{x \rightarrow \infty} Q_{1,1,2}(x, c)=\lim _{x \rightarrow \infty} \frac{\mathbb{P}\left(X_{0}>c^{2 / 3} x^{2 / 3}\right)}{\mathbb{P}\left(X_{0}>x^{2 / 3}\right)}=c^{-2 \alpha / 3},
$$

since $X_{0}$ is regularly varying with tail index $\alpha$. For each $c \in(0,1)$, using $X_{0}^{3 / 2} \vee X_{0}\left|M_{1}\right|=$ $X_{0}^{3 / 2}\left(1 \vee \frac{\left|M_{1}\right|}{\sqrt{X_{0}}}\right)$, if $X_{0}>0$, and that (2.2) yields $\mathcal{L}\left(x^{-1} X_{0}, \frac{M_{1}}{\sqrt{X_{0}}} \mid X_{0}>x\right) \stackrel{w}{\longrightarrow} \mathcal{L}\left(Y_{0}, Z_{0}\right)$ as $x \rightarrow \infty$ (since $X_{0} \vee 1=X_{0}$ if $X_{0}>1$ ), by Lemma D.3, we obtain

$$
\begin{aligned}
Q_{1,1,1}(x, c) & =\mathbb{P}\left(\left(X_{0}^{3 / 2} \vee X_{0}\left|M_{1}\right|\right)>x \mid X_{0}^{3 / 2}>c x\right) \\
& =\mathbb{P}\left(\left(\frac{X_{0}}{(c x)^{2 / 3}}\right)^{3 / 2}\left(1 \vee \frac{\left|M_{1}\right|}{\sqrt{X_{0}}}\right)>\frac{1}{c} \mid X_{0}>(c x)^{2 / 3}\right) \\
& \rightarrow \mathbb{P}\left(Y_{0}^{3 / 2}\left(1 \vee\left|Z_{0}\right|\right)>c^{-1}\right) \quad \text { as } x \rightarrow \infty,
\end{aligned}
$$

where $Y_{0}$ is a Pareto distributed random variable such that $\mathbb{P}\left(Y_{0} \geq y\right)=y^{-\alpha}, y \geq 1$. Consequently, for each $c \in(0,1)$, we have

$$
Q_{1,1}(x, c) \rightarrow c^{-2 \alpha / 3} \mathbb{P}\left(Y_{0}^{3 / 2}\left(1 \vee\left|Z_{0}\right|\right)>c^{-1}\right) \quad \text { as } x \rightarrow \infty .
$$


For each $c \in(0,1)$, by the tower rule and using $\mathbb{P}\left(Y_{0}>y\right)=y^{-\alpha} \wedge 1, y \in(0, \infty)$, we have

$$
\begin{aligned}
& c^{-2 \alpha / 3} \mathbb{P}\left(Y_{0}^{3 / 2}\left(1 \vee\left|Z_{0}\right|\right)>c^{-1}\right) \\
& =c^{-2 \alpha / 3} \mathbb{E}\left(\mathbb{P}\left(Y_{0}^{3 / 2}\left(1 \vee\left|Z_{0}\right|\right)>c^{-1} \mid Z_{0}\right)\right) \\
& =c^{-2 \alpha / 3} \mathbb{E}\left(\mathbb{P}\left(Y_{0}>c^{-2 / 3}\left(1 \vee\left|Z_{0}\right|\right)^{-2 / 3} \mid Z_{0}\right)\right) \\
& =c^{-2 \alpha / 3} \mathbb{E}\left(\left(c^{-2 / 3}\left(1 \vee\left|Z_{0}\right|\right)^{-2 / 3}\right)^{-\alpha} \wedge 1\right) \\
& =\mathbb{E}\left(\left(1 \vee\left|Z_{0}\right|\right)^{2 \alpha / 3} \wedge c^{-2 \alpha / 3}\right),
\end{aligned}
$$

hence we have $\lim _{c \downarrow 0} \lim _{x \rightarrow \infty} Q_{1,1}(x, c)=\mathbb{E}\left(\left(1 \vee\left|Z_{0}\right|\right)^{2 \alpha / 3}\right)$. Moreover, for each $x \in(0, \infty)$ and $c \in(0,1)$, we have $Q_{1,2}(x, c)=Q_{1,2,1}(x, c) Q_{1,1,2}(x, c)$ with

$$
\begin{aligned}
Q_{1,2,1}(x, c) & :=\frac{\mathbb{P}\left(\left(X_{0}^{3 / 2} \vee X_{0}\left|M_{1}\right|\right)>x, X_{0}^{3 / 2} \leq c x\right)}{\mathbb{P}\left(X_{0}^{3 / 2}>c x\right)}=\frac{\mathbb{P}\left(X_{0}\left|M_{1}\right|>x, X_{0}^{3 / 2} \leq c x\right)}{\mathbb{P}\left(X_{0}^{3 / 2}>c x\right)} \\
& =\frac{\mathbb{P}\left(\left|M_{1}\right| X_{0} \mathbb{1}_{\left\{X_{0}^{3 / 2} \leq c x\right\}}>x\right)}{\mathbb{P}\left(X_{0}^{3 / 2}>c x\right)} .
\end{aligned}
$$

By (E.5), we have $\left(X_{0}, M_{1}\right) \stackrel{d}{=}\left(X_{0}, \widetilde{\kappa}_{1}+\widetilde{\theta}_{1}^{(0)} \circ X_{0}\right)$. Hence, for each $x \in(0, \infty), c \in(0,1)$ and $\delta \in\left(0, \frac{\alpha}{3}\right)$, by Markov's inequality and the independence of $\widetilde{\kappa}_{1}$ and $X_{0}$,

$$
\begin{aligned}
& \mathbb{P}\left(\left|M_{1}\right| X_{0} \mathbb{1}_{\left\{X_{0}^{3 / 2} \leq c x\right\}}>x\right) \\
& \leq \mathbb{P}\left(\left|\widetilde{\kappa}_{1}\right| X_{0} \mathbb{1}_{\left\{X_{0}^{3 / 2} \leq c x\right\}}>\frac{x}{2}\right)+\mathbb{P}\left(\left|\widetilde{\theta}_{1}^{(0)} \circ X_{0}\right| X_{0} \mathbb{1}_{\left\{X_{0}^{3 / 2} \leq c x\right\}}>\frac{x}{2}\right) \\
& \leq\left(\frac{2}{x}\right)^{\alpha-\delta} \mathbb{E}\left(\left|\widetilde{\kappa}_{1}\right|^{\alpha-\delta}\right) \mathbb{E}\left(X_{0}^{\alpha-\delta} \mathbb{1}_{\left\{X_{0}^{3 / 2} \leq c x\right\}}\right)+\left(\frac{2}{x}\right)^{2} \mathbb{E}\left(\left(\widetilde{\theta}_{1}^{(0)} \circ X_{0}\right)^{2} X_{0}^{2} \mathbb{1}_{\left\{X_{0}^{3 / 2} \leq c x\right\}}\right) .
\end{aligned}
$$

We have $\mathbb{E}\left(\left|\widetilde{\kappa}_{1}\right|^{\alpha-\delta}\right)=\mathbb{E}\left(\left|B-\mu_{B}\right|^{\alpha-\delta}\right)<\infty$, since $\left|B-\mu_{B}\right|$ is regularly varying with tail index $\alpha$, see (4.6). Moreover, $\mathbb{E}\left(X_{0}^{\alpha-\delta} \mathbb{1}_{\left\{X_{0}^{3 / 2} \leq c x\right\}}\right) \leq \mathbb{E}\left(X_{0}^{\alpha-\delta}\right)<\infty$, since $X_{0}$ is regularly varying with tail index $\alpha$. Further,

$$
\mathbb{E}\left(\left(\widetilde{\theta}_{1}^{(0)} \circ X_{0}\right)^{2} X_{0}^{2} \mathbb{1}_{\left\{X_{0}^{3 / 2} \leq c x\right\}}\right)=\mathbb{E}\left(\mathbb{E}\left(\left(\widetilde{\theta}_{1}^{(0)} \circ X_{0}\right)^{2} X_{0}^{2} \mathbb{1}_{\left\{X_{0}^{3 / 2} \leq c x\right\}} \mid X_{0}\right)\right)=\sigma_{A}^{2} \mathbb{E}\left(X_{0}^{3} \mathbb{1}_{\left\{X_{0}^{3 / 2} \leq c x\right\}}\right) .
$$

Consequently, for each $x \in(0, \infty), c \in(0,1)$ and $\delta \in\left(0, \frac{\alpha}{3}\right)$,

$$
Q_{1,2,1}(x, c) \leq \frac{2^{\alpha-\delta} \mathbb{E}\left(\left|B-\mu_{B}\right|^{\alpha-\delta}\right) \mathbb{E}\left(X_{0}^{\alpha-\delta}\right)}{x^{\alpha-\delta} \mathbb{P}\left(X_{0}^{3 / 2}>c x\right)}+4 \sigma_{A}^{2} \frac{\mathbb{E}\left(X_{0}^{3} \mathbb{1}_{\left\{X_{0}^{3 / 2} \leq c x\right\}}\right)}{x^{2} \mathbb{P}\left(X_{0}^{3 / 2}>c x\right)}
$$

The random variable $X_{0}^{3 / 2}$ is regularly varying with tail index $\frac{2 \alpha}{3}$, since $X_{0}$ is regularly varying with tail index $\alpha$. Hence $\alpha-\delta>\frac{2 \alpha}{3}$ yields $x^{\alpha-\delta} \mathbb{P}\left(X_{0}^{3 / 2}>c x\right) \rightarrow \infty$ as $x \rightarrow \infty$ (see, e.g., Bingham et al. [9, Proposition 1.3.6. (v)]). Applying Karamata's theorem (see, Lemma E.1), we obtain

$$
\frac{\mathbb{E}\left(X_{0}^{3} \mathbb{1}_{\left\{X_{0}^{3 / 2} \leq c x\right\}}\right)}{x^{2} \mathbb{P}\left(X_{0}^{3 / 2}>c x\right)}=c^{2} \frac{\mathbb{E}\left(\left(X_{0}^{3 / 2}\right)^{2} \mathbb{1}_{\left\{X_{0}^{3 / 2} \leq c x\right\}}\right)}{(c x)^{2} \mathbb{P}\left(X_{0}^{3 / 2}>c x\right)} \rightarrow c^{2} \frac{\frac{2 \alpha}{3}}{2-\frac{2 \alpha}{3}}=\frac{c^{2} \alpha}{3-\alpha}
$$


as $x \rightarrow \infty$. Consequently, by (E.7), for each $c \in(0,1)$, we obtain

$$
\limsup _{x \rightarrow \infty} Q_{1,2}(x, c) \leq 4 c^{-2 \alpha / 3} \sigma_{A}^{2} \frac{c^{2} \alpha}{3-\alpha}=\frac{4 \alpha \sigma_{A}^{2}}{3-\alpha} c^{2(3-\alpha) / 3},
$$

hence

$$
\lim _{c \downarrow 0} \liminf _{x \rightarrow \infty} Q_{1,2}(x, c)=\lim _{c \downarrow 0} \limsup _{x \rightarrow \infty} Q_{1,2}(x, c)=0 .
$$

Summarizing, we get

$$
Q_{1}(x) \rightarrow \mathbb{E}\left(\left(1 \vee\left|Z_{0}\right|\right)^{2 \alpha / 3}\right) \quad \text { as } x \rightarrow \infty
$$

Now we consider the term $P_{1}(x, y), x, y \in(0, \infty)$. For each $x, y \in(0, \infty)$, we have $P_{1}(x, y)=$ $P_{1,1}(x, y) P_{1,2}(x, y)$, where

$$
\begin{aligned}
& P_{1,1}(x, y):=\frac{\mathbb{P}\left(X_{0}^{3 / 2}>x y\right)}{\mathbb{P}\left(X_{0}^{3 / 2}>x\right)}, \\
& P_{1,2}(x, y):=\frac{\mathbb{P}\left(X_{0}^{3 / 2}>x y,\left(X_{0}^{3 / 2} \vee X_{0}\left|M_{1}\right|\right)>x\right)}{\mathbb{P}\left(X_{0}^{3 / 2}>x y\right)} .
\end{aligned}
$$

Since $X_{0}$ is regularly varying with tail index $\alpha$, for each $y \in(0, \infty)$, we have $\lim _{x \rightarrow \infty} P_{1,1}(x, y)=$ $y^{-2 \alpha / 3}$. Further, for each $y \in(0, \infty)$, using that $(2.2)$ yields $\mathcal{L}\left(x^{-1} X_{0}, \frac{M_{1}}{\sqrt{X_{0}}} \mid X_{0}>x\right) \stackrel{w}{\longrightarrow}$ $\mathcal{L}\left(Y_{0}, Z_{0}\right)$ as $x \rightarrow \infty$ (since $X_{0} \vee 1=X_{0}$ if $X_{0}>1$ ), by Lemma D.3, we have

$$
\begin{aligned}
P_{1,2}(x, y) & =\mathbb{P}\left(\left(X_{0}^{3 / 2} \vee X_{0}\left|M_{1}\right|\right)>x \mid X_{0}^{3 / 2}>x y\right) \\
& =\mathbb{P}\left(\left(\frac{X_{0}}{(x y)^{2 / 3}}\right)^{3 / 2}\left(1 \vee \frac{\left|M_{1}\right|}{\sqrt{X_{0}}}\right)>\frac{1}{y} \mid X_{0}>(x y)^{2 / 3}\right) \\
& \rightarrow \mathbb{P}\left(Y_{0}^{3 / 2}\left(1 \vee\left|Z_{0}\right|\right)>y^{-1}\right) \quad \text { as } x \rightarrow \infty
\end{aligned}
$$

Consequently, for each $y \in(0, \infty)$, by the tower rule and using $\mathbb{P}\left(Y_{0}>y\right)=y^{-\alpha} \wedge 1, y \in(0, \infty)$, we have

$$
\begin{aligned}
P_{1}(x, y) & \rightarrow y^{-2 \alpha / 3} \mathbb{P}\left(Y_{0}^{3 / 2}\left(1 \vee\left|Z_{0}\right|\right)>y^{-1}\right)=y^{-2 \alpha / 3} \mathbb{P}\left(Y_{0}>\left(y\left(1 \vee\left|Z_{0}\right|\right)\right)^{-2 / 3}\right) \\
& =y^{-2 \alpha / 3} \mathbb{E}\left[\mathbb{P}\left(Y_{0}>\left(y\left(1 \vee\left|Z_{0}\right|\right)\right)^{-2 / 3} \mid Z_{0}\right)\right]=y^{-2 \alpha / 3} \mathbb{E}\left[\left(y^{2 \alpha / 3}\left(1 \vee\left|Z_{0}\right|\right)^{2 \alpha / 3}\right) \wedge 1\right] \\
& =\mathbb{E}\left[\left(1 \vee\left|Z_{0}\right|\right)^{2 \alpha / 3} \wedge y^{-2 \alpha / 3}\right]=\mathbb{E}\left[\left(y \vee\left(1 \vee\left|Z_{0}\right|\right)^{-1}\right)^{-2 \alpha / 3}\right] \quad \text { as } x \rightarrow \infty .
\end{aligned}
$$

By (E.10), for each $y \in(0, \infty)$, we obtain

$$
\mathbb{P}\left(\frac{X_{0}^{3 / 2}}{x}>y \mid\left(X_{0}^{3 / 2} \vee X_{0}\left|M_{1}\right|\right)>x\right) \rightarrow \frac{\mathbb{E}\left(\left(y \vee\left(1 \vee\left|Z_{0}\right|\right)^{-1}\right)^{-2 \alpha / 3}\right)}{\mathbb{E}\left(\left(1 \vee\left|Z_{0}\right|\right)^{2 \alpha / 3}\right)}
$$

as $x \rightarrow \infty$, thus we conclude (E.6). 
Step 2. We check that for each $k \in \mathbb{N}$,

$$
\mathcal{L}\left(\left(\frac{X_{k}}{X_{0}}\right)^{3 / 2} \mid\left(X_{0}^{3 / 2} \vee X_{0}\left|M_{1}\right|\right)>x\right) \stackrel{w}{\longrightarrow} \delta_{\mu_{A}^{3 k / 2}} \quad \text { as } x \rightarrow \infty .
$$

For each $k \in \mathbb{N}, x, y \in(0, \infty)$, we have

$$
\mathbb{P}\left(\left(\frac{X_{k}}{X_{0}}\right)^{3 / 2}>y \mid\left(X_{0}^{3 / 2} \vee X_{0}\left|M_{1}\right|\right)>x\right)=\frac{P_{2}(x, y)}{Q_{1}(x)}
$$

with

$$
P_{2}(x, y):=\frac{\mathbb{P}\left(\left(\frac{X_{k}}{X_{0}}\right)^{3 / 2}>y,\left(X_{0}^{3 / 2} \vee X_{0}\left|M_{1}\right|\right)>x\right)}{\mathbb{P}\left(X_{0}^{3 / 2}>x\right)} .
$$

For each $k \in \mathbb{N}, x, y \in(0, \infty)$, and $c \in(0,1)$, we can write $P_{2}(x, y)=P_{2,1}(x, y, c)+P_{2,2}(x, y, c)$ with

$$
\begin{aligned}
P_{2,1}(x, y, c) & :=\frac{\mathbb{P}\left(\left(\frac{X_{k}}{X_{0}}\right)^{3 / 2}>y,\left(X_{0}^{3 / 2} \vee X_{0}\left|M_{1}\right|\right)>x, X_{0}^{3 / 2}>c x\right)}{\mathbb{P}\left(X_{0}^{3 / 2}>x\right)}, \\
P_{2,2}(x, y, c) & :=\frac{\mathbb{P}\left(\left(\frac{X_{k}}{X_{0}}\right)^{3 / 2}>y,\left(X_{0}^{3 / 2} \vee X_{0}\left|M_{1}\right|\right)>x, X_{0}^{3 / 2} \leq c x\right)}{\mathbb{P}\left(X_{0}^{3 / 2}>x\right)},
\end{aligned}
$$

where $P_{2,1}(x, y, c)=P_{2,1,1}(x, y, c) Q_{1,1,2}(x, c)$ with

$$
P_{2,1,1}(x, y, c):=\frac{\mathbb{P}\left(\left(\frac{X_{k}}{X_{0}}\right)^{3 / 2}>y,\left(X_{0}^{3 / 2} \vee X_{0}\left|M_{1}\right|\right)>x, X_{0}^{3 / 2}>c x\right)}{\mathbb{P}\left(X_{0}^{3 / 2}>c x\right)} .
$$

For each $k \in \mathbb{N}, y \in(0, \infty) \backslash\left\{\mu_{A}^{3 k / 2}\right\}$ and $c \in(0,1)$, using (2.1), (2.2) and Lemma D.3, we obtain

$$
\begin{aligned}
& P_{2,1,1}(x, y, c)=\mathbb{P}\left(\left(\frac{X_{k}}{X_{0}}\right)^{3 / 2}>y,\left(X_{0}^{3 / 2} \vee X_{0}\left|M_{1}\right|\right)>x \mid X_{0}^{3 / 2}>c x\right) \\
& =\mathbb{P}\left(\left(\frac{(c x)^{-2 / 3} X_{k}}{(c x)^{-2 / 3} X_{0}}\right)^{3 / 2}>y,\left(\frac{X_{0}}{(c x)^{2 / 3}}\right)^{3 / 2}\left(1 \vee \frac{\left|M_{1}\right|}{\sqrt{X_{0}}}\right)>\frac{1}{c} \mid X_{0}>(c x)^{2 / 3}\right) \\
& \rightarrow \mathbb{P}\left(\mu_{A}^{3 k / 2}>y, Y_{0}^{3 / 2}\left(1 \vee\left|Z_{0}\right|\right)>c^{-1}\right) \quad \text { as } x \rightarrow \infty .
\end{aligned}
$$

Consequently, by (E.7) and (E.8), for each $k \in \mathbb{N}, y \in(0, \infty) \backslash\left\{\mu_{A}^{3 k / 2}\right\}$ and $c \in(0,1)$, we have

$$
\begin{aligned}
P_{2,1}(x, y, c) & \rightarrow c^{-2 \alpha / 3} \mathbb{1}_{(y, \infty)}\left(\mu_{A}^{3 k / 2}\right) \mathbb{P}\left(Y_{0}^{3 / 2}\left(1 \vee\left|Z_{0}\right|\right)>c^{-1}\right) \\
& =\mathbb{1}_{(y, \infty)}\left(\mu_{A}^{3 k / 2}\right) \mathbb{E}\left(\left(1 \vee\left|Z_{0}\right|\right)^{2 \alpha / 3} \wedge c^{-2 \alpha / 3}\right) \quad \text { as } x \rightarrow \infty,
\end{aligned}
$$

hence, for each $k \in \mathbb{N}$ and $y \in(0, \infty) \backslash\left\{\mu_{A}^{3 k / 2}\right\}$, we have

$$
\lim _{c \downarrow 0} \lim _{x \rightarrow \infty} P_{2,1}(x, y, c)=\mathbb{1}_{(y, \infty)}\left(\mu_{A}^{3 k / 2}\right) \mathbb{E}\left(\left(1 \vee\left|Z_{0}\right|\right)^{2 \alpha / 3}\right) .
$$


Moreover, for each $x, y \in(0, \infty)$ and $c \in(0,1)$, we have $P_{2,2}(x, y, c) \leq Q_{1,2}(x, c)$, hence, by (E.9), $\lim _{c \downarrow 0} \liminf \operatorname{in}_{x \rightarrow \infty} P_{2,2}(x, y, c)=\lim _{c \downarrow 0} \limsup _{x \rightarrow \infty} P_{2,2}(x, y, c)=0$. Summarizing, for each $k \in \mathbb{N}$ and $y \in(0, \infty) \backslash\left\{\mu_{A}^{3 k / 2}\right\}$, we get

$$
P_{2}(x, y) \rightarrow \mathbb{1}_{(y, \infty)}\left(\mu_{A}^{3 k / 2}\right) \mathbb{E}\left(\left(1 \vee\left|Z_{0}\right|\right)^{2 \alpha / 3}\right) \quad \text { as } x \rightarrow \infty
$$

By (E.10), for each $k \in \mathbb{N}$ and $y \in(0, \infty) \backslash\left\{\mu_{A}^{3 k / 2}\right\}$, we obtain

$$
\mathbb{P}\left(\left(\frac{X_{k}}{X_{0}}\right)^{3 / 2}>y \mid\left(X_{0}^{3 / 2} \vee X_{0}\left|M_{1}\right|\right)>x\right) \rightarrow \mathbb{1}_{(y, \infty)}\left(\mu_{A}^{3 k / 2}\right) \quad \text { as } x \rightarrow \infty,
$$

thus we conclude (E.11).

Step 3. We check that for each $n \geq 0$,

$$
\mathcal{L}\left(\frac{X_{0}^{3 / 2}}{x}, \frac{M_{1}}{\sqrt{X_{0}}}, \ldots, \frac{M_{n+1}}{\sqrt{X_{0}}} \mid\left(X_{0}^{3 / 2} \vee X_{0}\left|M_{1}\right|\right)>x\right) \stackrel{d}{\longrightarrow} \mathcal{L}\left(\widetilde{Y}, \widetilde{Z}_{0}, \mu_{A}^{1 / 2} \widetilde{Z}_{1}, \ldots, \mu_{A}^{n / 2} \widetilde{Z}_{n}\right)
$$

as $x \rightarrow \infty$. For each $n \geq 0, x, y \in(0, \infty)$ and $v_{0}, \ldots, v_{n} \in \mathbb{R}$, we have

$$
\mathbb{P}\left(\frac{X_{0}^{3 / 2}}{x}>y, \frac{M_{1}}{\sqrt{X_{0}}}>v_{0}, \ldots, \frac{M_{n+1}}{\sqrt{X_{0}}}>v_{n} \mid\left(X_{0}^{3 / 2} \vee X_{0}\left|M_{1}\right|\right)>x\right)=\frac{P_{3}\left(x, y, v_{0}, \ldots, v_{n}\right)}{Q_{1}(x)}
$$

with

$$
P_{3}\left(x, y, v_{0}, \ldots, v_{n}\right):=\frac{\mathbb{P}\left(\frac{X_{0}^{3 / 2}}{x}>y, \frac{M_{1}}{\sqrt{X_{0}}}>v_{0}, \ldots, \frac{M_{n+1}}{\sqrt{X_{0}}}>v_{n},\left(X_{0}^{3 / 2} \vee X_{0}\left|M_{1}\right|\right)>x\right)}{\mathbb{P}\left(X_{0}^{3 / 2}>x\right)}
$$

For each $n \geq 0, x, y \in(0, \infty)$, and $v_{0}, \ldots, v_{n} \in \mathbb{R}$, we can write $P_{3}\left(x, y, v_{0}, \ldots, v_{n}\right)=$ $P_{3,1}\left(x, y, v_{0}, \ldots, v_{n}\right) P_{3,2}(x, y)$ with

$$
\begin{aligned}
P_{3,1}\left(x, y, v_{0}, \ldots, v_{n}\right) & :=\frac{\mathbb{P}\left(\frac{X_{0}^{3 / 2}}{x}>y, \frac{M_{1}}{\sqrt{X_{0}}}>v_{0}, \ldots, \frac{M_{n+1}}{\sqrt{X_{0}}}>v_{n},\left(X_{0}^{3 / 2} \vee X_{0}\left|M_{1}\right|\right)>x\right)}{\mathbb{P}\left(X_{0}^{3 / 2}>y x\right)} \\
& =\mathbb{P}\left(\left(X_{0}^{3 / 2} \vee X_{0}\left|M_{1}\right|\right)>x, \frac{M_{1}}{\sqrt{X_{0}}}>v_{0}, \ldots, \frac{M_{n+1}}{\sqrt{X_{0}}}>v_{n} \mid X_{0}^{3 / 2}>y x\right), \\
P_{3,2}(x, y) & :=\frac{\mathbb{P}\left(X_{0}^{3 / 2}>y x\right)}{\mathbb{P}\left(X_{0}^{3 / 2}>x\right)} .
\end{aligned}
$$

Since $X_{0}^{3 / 2}$ is regularly varying with tail index $2 \alpha / 3$, we have for each $y \in(0, \infty)$,

$$
\lim _{x \rightarrow \infty} P_{3,2}(x, y)=y^{-2 \alpha / 3}
$$

Further, using that (2.2) holds with $W_{i}^{\prime}$ replaced by $W_{i}$ (see the proof of Proposition 2.1), by 
Lemma D.3, we have

$$
\begin{aligned}
& P_{3,1}\left(x, y, v_{0}, \ldots, v_{n}\right) \\
& =\mathbb{P}\left(\left(\frac{X_{0}}{(y x)^{2 / 3}}\right)^{3 / 2}\left(1 \vee \frac{\left|M_{1}\right|}{\sqrt{X_{0}}}\right)>\frac{1}{y},\right. \\
& \left.\frac{M_{1}}{\sqrt{X_{0}}}>v_{0}, \frac{M_{2}}{\sqrt{X_{0} \mu_{A}}}>\frac{v_{1}}{\sqrt{\mu_{A}}}, \ldots, \frac{M_{n+1}}{\sqrt{X_{0} \mu_{A}^{n}}}>\frac{v_{n}}{\sqrt{\mu_{A}^{n}}} \mid X_{0}>(y x)^{2 / 3}\right) \\
& \rightarrow \mathbb{P}\left(Y_{0}^{3 / 2}\left(1 \vee\left|Z_{0}\right|\right)>y^{-1}, Z_{0}>v_{0}, Z_{1}>\mu_{A}^{-1 / 2} v_{1}, \ldots, Z_{n}>\mu_{A}^{-n / 2} v_{n}\right) \\
& =\mathbb{P}\left(Y_{0}^{3 / 2}\left(1 \vee\left|Z_{0}\right|\right)>y^{-1}, Z_{0}>v_{0}\right) \mathbb{P}\left(Z_{1}>\mu_{A}^{-1 / 2} v_{1}, \ldots, Z_{n}>\mu_{A}^{-n / 2} v_{n}\right)
\end{aligned}
$$

as $x \rightarrow \infty$. Consequently, for each $n \geq 0, y \in(0, \infty)$ and $v_{0}, \ldots, v_{n} \in \mathbb{R}$, we get

$$
\begin{aligned}
& \lim _{x \rightarrow \infty} P_{3}\left(x, y, v_{0}, \ldots, v_{n}\right) \\
& =y^{-2 \alpha / 3} \mathbb{P}\left(Y_{0}^{3 / 2}\left(1 \vee\left|Z_{0}\right|\right)>y^{-1}, Z_{0}>v_{0}\right) \mathbb{P}\left(Z_{1}>\mu_{A}^{-1 / 2} v_{1}, \ldots, Z_{n}>\mu_{A}^{-n / 2} v_{n}\right) \\
& =y^{-2 \alpha / 3} \mathbb{E}\left[\mathbb{P}\left(Y_{0}^{3 / 2}\left(1 \vee\left|Z_{0}\right|\right)>y^{-1}, Z_{0}>v_{0} \mid Z_{0}\right)\right] \mathbb{P}\left(Z_{1}>\mu_{A}^{-1 / 2} v_{1}, \ldots, Z_{n}>\mu_{A}^{-n / 2} v_{n}\right) \\
& =y^{-2 \alpha / 3} \mathbb{E}\left[\mathbb{1}_{\left\{Z_{0}>v_{0}\right\}} \mathbb{P}\left(Y_{0}>\left(y\left(1 \vee\left|Z_{0}\right|\right)\right)^{-2 / 3} \mid Z_{0}\right)\right] \mathbb{P}\left(Z_{1}>\mu_{A}^{-1 / 2} v_{1}, \ldots, Z_{n}>\mu_{A}^{-n / 2} v_{n}\right) \\
& =y^{-2 \alpha / 3} \mathbb{E}\left[\mathbb{1}_{\left\{Z_{0}>v_{0}\right\}}\left(\left(y\left(1 \vee\left|Z_{0}\right|\right)\right)^{2 \alpha / 3} \wedge 1\right)\right] \mathbb{P}\left(Z_{1}>\mu_{A}^{-1 / 2} v_{1}, \ldots, Z_{n}>\mu_{A}^{-n / 2} v_{n}\right) \\
& =\mathbb{E}\left[\mathbb{1}_{\left\{Z_{0}>v_{0}\right\}}\left(\left(1 \vee\left|Z_{0}\right|\right)^{2 \alpha / 3} \wedge y^{-2 \alpha / 3}\right)\right] \mathbb{P}\left(Z_{1}>\mu_{A}^{-1 / 2} v_{1}, \ldots, Z_{n}>\mu_{A}^{-n / 2} v_{n}\right) \\
& =\mathbb{E}\left(\left(y \vee\left(1 \vee\left|Z_{0}\right|\right)^{-1}\right)^{-2 \alpha / 3} \mathbb{1}_{\left(v_{0}, \infty\right)}\left(Z_{0}\right)\right) \mathbb{P}\left(\mu_{A}^{1 / 2} Z_{1}>v_{1}, \ldots, \mu_{A}^{n / 2} Z_{n}>v_{n}\right) .
\end{aligned}
$$

By (E.10), for each $n \geq 0, y \in(0, \infty)$ and $v_{0}, \ldots, v_{n} \in \mathbb{R}$, we obtain

$$
\begin{aligned}
& \mathbb{P}\left(\frac{X_{0}^{3 / 2}}{x}>y, \frac{M_{1}}{\sqrt{X_{0}}}>v_{0}, \ldots, \frac{M_{n+1}}{\sqrt{X_{0}}}>v_{n} \mid\left(X_{0}^{3 / 2} \vee X_{0}\left|M_{1}\right|\right)>x\right) \\
& \rightarrow \frac{\mathbb{E}\left(\left(y \vee\left(1 \vee\left|Z_{0}\right|\right)^{-1}\right)^{-2 \alpha / 3} \mathbb{1}_{\left(v_{0}, \infty\right)}\left(Z_{0}\right)\right)}{\mathbb{E}\left(\left(1 \vee\left|Z_{0}\right|\right)^{2 \alpha / 3}\right)} \mathbb{P}\left(\mu_{A}^{1 / 2} Z_{1}>v_{1}, \ldots, \mu_{A}^{n / 2} Z_{n}>v_{n}\right) \\
& =\mathbb{P}\left(\widetilde{Y}>y, \widetilde{Z}_{0}>v_{0}\right) \mathbb{P}\left(\mu_{A}^{1 / 2} \widetilde{Z}_{1}>v_{1}, \ldots, \mu_{A}^{n / 2} \widetilde{Z}_{n}>v_{n}\right) \\
& =\mathbb{P}\left(\widetilde{Y}>y, \widetilde{Z}_{0}>v_{0}, \mu_{A}^{1 / 2} \widetilde{Z}_{1}>v_{1}, \ldots, \mu_{A}^{n / 2} \widetilde{Z}_{n}>v_{n}\right)
\end{aligned}
$$

as $x \rightarrow \infty$, thus we conclude (E.12).

Step 4. For all $n \geq 0$, we have

$$
\begin{aligned}
& \mathcal{L}\left(\left(\frac{1}{x}\left(X_{k}^{3 / 2}, X_{k} M_{k+1}\right)\right)_{k \in\{0,1, \ldots, n\}} \mid\left(X_{0}^{3 / 2} \vee X_{0}\left|M_{1}\right|\right)>x\right) \\
& \left.=\mathcal{L}\left(\left(\frac{X_{0}^{3 / 2}}{x}\left(\frac{X_{k}}{X_{0}}\right)^{3 / 2}, \frac{X_{0}^{3 / 2}}{x} \frac{X_{k}}{X_{0}} \frac{M_{k+1}}{\sqrt{X_{0}}}\right)\right)_{k \in\{0,1, \ldots, n\}} \mid\left(X_{0}^{3 / 2} \vee X_{0}\left|M_{1}\right|\right)>x\right)
\end{aligned}
$$




$$
\stackrel{d}{\longrightarrow} \mathcal{L}\left(\left(\widetilde{Y} \mu_{A}^{3 k / 2}, \widetilde{Y} \mu_{A}^{k} \mu_{A}^{k / 2} \widetilde{Z}_{k}\right)_{k \in\{0,1, \ldots, n\}}\right) \quad \text { as } x \rightarrow \infty .
$$

Indeed, (E.11), Lemmas D.3 and D.4 yield

$$
\mathcal{L}\left(\left(\frac{X_{k}}{X_{0}}\right)_{k \in\{0,1, \ldots, n\}} \mid\left(X_{0}^{3 / 2} \vee X_{0}\left|M_{1}\right|\right)>x\right) \stackrel{w}{\longrightarrow} \delta_{\left(1, \mu_{A}, \ldots, \mu_{A}^{n}\right)} \quad \text { as } x \rightarrow \infty
$$

and then, identifying $\mathbb{R}^{(2 n+2) \times(2 n+2)}$ with $\mathbb{R}^{(2 n+2)^{2}}$ in a natural way, we can use again Lemma D.3 to obtain

$$
\begin{gathered}
\mathcal{L}\left(\operatorname{diag}_{2 n+2}\left(1,1,\left(\frac{X_{1}}{X_{0}}\right)^{3 / 2}, \frac{X_{1}}{X_{0}}, \ldots,\left(\frac{X_{n}}{X_{0}}\right)^{3 / 2}, \frac{X_{n}}{X_{0}}\right) \mid\left(X_{0}^{3 / 2} \vee X_{0}\left|M_{1}\right|\right)>x\right) \\
\stackrel{w}{\longrightarrow} \delta_{\operatorname{diag}_{2 n+2}\left(1,1, \mu_{A}^{3 / 2}, \mu_{A}, \ldots, \mu_{A}^{3 n / 2}, \mu_{A}^{n}\right)} \quad \text { as } x \rightarrow \infty .
\end{gathered}
$$

Next, (E.12) and the conditional version of the continuous mapping theorem (see Lemma D.3) imply

$$
\begin{aligned}
& \left.\mathcal{L}\left(\left(\left(\frac{X_{0}}{x^{2 / 3}}\right)^{3 / 2},\left(\frac{X_{0}}{x^{2 / 3}}\right)^{3 / 2} \frac{M_{k+1}}{\sqrt{X_{0}}}\right)\right)_{k \in\{0,1, \ldots, n\}} \mid\left(X_{0}^{3 / 2} \vee X_{0}\left|M_{1}\right|\right)>x\right) \\
& \stackrel{w}{\longrightarrow} \mathcal{L}\left(\left(\widetilde{Y}^{3 / 2}, \widetilde{Y}^{3 / 2} \mu_{A}^{k / 2} \widetilde{Z}_{k}\right)_{k \in\{0,1, \ldots, n\}}\right) \quad \text { as } x \rightarrow \infty .
\end{aligned}
$$

Finally, identifying $\mathbb{R}^{n+1} \times \mathbb{R}^{n+1}$ and $\mathbb{R}^{2(n+1)}$ in a natural way and applying Lemma D.5, we obtain the convergence statement of the theorem.

The jointly regularly varying property of $\left(X_{k}^{3 / 2}, X_{k} M_{k+1}\right)_{k \in \mathbb{Z}}$ follows by Theorem 2.1 in Basrak and Segers [7]. The existence of a (whole) tail process of $\left(X_{k}^{3 / 2}, X_{k} M_{k+1}\right)_{k \in \mathbb{Z}}$ follows by Basrak and Segers [7, Theorem 2.1].

In the next remark we point out that $\tilde{Y}$ given in Theorem E.3 is not a Pareto-distributed random variable.

Remark E.4. Note that (E.3) readily yields that

$$
\mathbb{P}(\widetilde{Y}>y)=\frac{\mathbb{E}\left(\left(y \vee\left(1 \vee\left|Z_{0}\right|\right)^{-1}\right)^{-2 \alpha / 3}\right)}{\mathbb{E}\left(\left(1 \vee\left|Z_{0}\right|\right)^{2 \alpha / 3}\right)}, \quad y \in \mathbb{R}
$$

Consequently, $\mathbb{P}(\tilde{Y} \in(0, \infty))=1$ and

$$
\mathbb{P}(\tilde{Y}>y) \leq \frac{1}{\mathbb{E}\left(\left(1 \vee\left|Z_{0}\right|\right)^{2 \alpha / 3}\right)} y^{-2 \alpha / 3} \quad \text { for } y \in(0, \infty),
$$

and equality holds for $y \in[1, \infty)$. Indeed, for each $y \in(0, \infty)$, we have $y \vee\left(1 \vee\left|Z_{0}\right|\right)^{-1} \geq y$ almost surely, thus $\left(y \vee\left(1 \vee\left|Z_{0}\right|\right)^{-1}\right)^{-2 \alpha / 3} \leq y^{-2 \alpha / 3}$ almost surely, hence $\mathbb{E}\left(\left(y \vee\left(1 \vee\left|Z_{0}\right|\right)^{-1}\right)^{-2 \alpha / 3}\right) \leq$ $y^{-2 \alpha / 3}$, and for $y \in[1, \infty)$, we have $y \vee\left(1 \vee\left|Z_{0}\right|\right)^{-1}=y$ almost surely. 


\section{Acknowledgements}

We are grateful to Márton Ispány for raising the problem in 2002 and to Peter Kern for calling attention to Corollary 2.1 in Łuczak [32] on the existence of an infinitely differentiable density function for operator stable laws. We would like to thank the referees for their comments that helped us to improve the paper. This paper has been revised after the sudden death of Gyula Pap, the fourth author and the main instigator of this project, in October 2019.

\section{References}

[1] Aloisio Araujo and Evarist Giné. The central limit theorem for real and Banach valued random variables. John Wiley \& Sons, New York-Chichester-Brisbane, 1980. Wiley Series in Probability and Mathematical Statistics.

[2] Mátyás Barczy, Fanni K. Nedényi, and Gyula Pap. On aggregation of multitype GaltonWatson branching processes with immigration. Mod. Stoch. Theory Appl., 5(1):53-79, 2018 .

[3] Mátyás Barczy, Fanni K. Nedényi, and Gyula Pap. Convergence of partial sum processes to stable processes with application for aggregation of branching processes. 2019. arXiv:1906.04999.

[4] Bojan Basrak. The sample autocorrelation function of non-linear time series. PhD thesis, Rijksuniversiteit Groningen Groningen, Netherlands, 2000.

[5] Bojan Basrak, Rafal Kulik, and Zbigniew Palmowski. Heavy-tailed branching process with immigration. Stoch. Models, 29(4):413-434, 2013.

[6] Bojan Basrak and Hrvoje Planinić. A note on vague convergence of measures. Statist. Probab. Lett., 153:180-186, 2019.

[7] Bojan Basrak and Johan Segers. Regularly varying multivariate time series. Stochastic Process. Appl., 119(4):1055-1080, 2009.

[8] Patrick Billingsley. Convergence of probability measures. John Wiley \& Sons, Inc., New York-London-Sydney, 1968.

[9] Nicholas H. Bingham, Charles M. Goldie, and Józef L. Teugels. Regular variation, volume 27 of Encyclopedia of Mathematics and its Applications. Cambridge University Press, Cambridge, 1987.

[10] Dariusz Buraczewski, Ewa Damek, and Thomas Mikosch. Stochastic models with powerlaw tails. The equation $X=A X+B$. Springer Series in Operations Research and Financial Engineering. Springer International Publishing, Switzerland, 2016. 
[11] Richard Davis and Tailen Hsing. Point process and partial sum convergence for weakly dependent random variables with infinite variance. Ann. Probab., 23(2):879-917, 1995.

[12] Richard Davis and Sidney Resnick. More limit theory for the sample correlation function of moving averages. Stochastic Process. Appl., 20(2):257-279, 1985.

[13] Richard Davis and Sidney Resnick. Limit theory for the sample covariance and correlation functions of moving averages. Ann. Statist., 14(2):533-558, 1986.

[14] Richard Durrett. Probability: theory and examples. Duxbury Press, Belmont, CA, second edition, 1996.

[15] Philip A. Ernst, Marek Kimmel, Monika Kurpas, and Quan Zhou. Heavy-tailed distributions in branching process models of secondary cancerous tumors. Adv. in Appl. Probab., 50(A):99-114, 2018.

[16] John Gurland. Inversion formulae for the distribution of ratios. Ann. Math. Statistics, 19:228-237, 1948.

[17] Allan Gut. Probability: a graduate course. Springer Texts in Statistics. Springer, New York, 2005.

[18] Edward James Hannan and Marek Kanter. Autoregressive processes with infinite variance. J. Appl. Probability, 14(2):411-415, 1977.

[19] Yaozhong Hu and Hongwei Long. Least squares estimator for Ornstein-Uhlenbeck processes driven by $\alpha$-stable motions. Stochastic Process. Appl., 119(8):2465-2480, 2009.

[20] Anja Janssen and Johan Segers. Markov tail chains. J. Appl. Probab., 51(4):1133-1153, 2014.

[21] Olav Kallenberg. Random measures, theory and applications, volume 77 of Probability Theory and Stochastic Modelling. Springer, Cham, 2017.

[22] Peter Kern and Lina Wedrich. The Hausdorff dimension of operator semistable Lévy processes. J. Theoret. Probab., 27(2):383-403, 2014.

[23] John F. C. Kingman. Poisson processes. The Clarendon Press, Oxford University Press, New York, 1993.

[24] Lawrence A. Klimko and Paul I. Nelson. On conditional least squares estimation for stochastic processes. Ann. Statist., 6(3):629-642, 1978.

[25] Daniel Krizmanić. Functional limit theorems for weakly dependent regularly varying time series. PhD thesis, University of Zagreb, 2010.

[26] Rafał Kulik and Philippe Soulier. Heavy tailed time series with extremal independence. Extremes, 18(2):273-299, 2015. 
[27] Rafał Kulik and Philippe Soulier. Heavy-Tailed Time Series. Springer Series in Operations Research and Financial Engineering. Springer-Verlag New York, 2020.

[28] Zenghu Li. Measure-valued branching Markov processes. Probability and its Applications (New York). Springer, Heidelberg, 2011.

[29] Zenghu Li and Chunhua Ma. Asymptotic properties of estimators in a stable Cox-IngersollRoss model. Stochastic Process. Appl., 125(8):3196-3233, 2015.

[30] Zhengyan Lin and Chuanrong Lu. Limit theory for mixing dependent random variables, volume 378 of Mathematics and its Applications. Kluwer Academic Publishers, Dordrecht; Science Press Beijing, New York, 1996.

[31] Filip Lindskog, Sidney I. Resnick, and Joyjit Roy. Regularly varying measures on metric spaces: hidden regular variation and hidden jumps. Probab. Surv., 11:270-314, 2014.

[32] Andrzej Łuczak. Operator semistable probability measures on $\mathbf{R}^{N}$. Colloq. Math., 45(2):287-300, 1981.

[33] Mark M. Meerschaert and Hans-Peter Scheffler. Limit distributions for sums of independent random vectors: Heavy tails in theory and practice. Wiley Series in Probability and Statistics: Probability and Statistics. John Wiley \& Sons, Inc., New York, 2001.

[34] Malcolm P. Quine. The multi-type Galton-Watson process with immigration. J. Appl. Probability, 7:411-422, 1970.

[35] Sidney I. Resnick. Extreme values, regular variation and point processes. Applied Probability, Vol. 4,. New York, Springer-Verlag, 1987.

[36] Sidney I. Resnick. Heavy-tail phenomena, Probabilistic and statistical modeling. Springer Series in Operations Research and Financial Engineering. Springer, New York, 2007.

[37] Gennady Samorodnitsky and Murad S. Taqqu. Stable non-Gaussian random processes: Stochastic models with infinite variance. Stochastic Modeling. Chapman \& Hall, New York, 1994.

[38] Ken-iti Sato. Lévy processes and infinitely divisible distributions, volume 68 of Cambridge Studies in Advanced Mathematics. Cambridge University Press, Cambridge, 2013.

[39] Hans-Jürgen Schuh and Andrew D. Barbour. On the asymptotic behaviour of branching processes with infinite mean. Advances in Appl. Probability, 9(4):681-723, 1977.

[40] Eugene Seneta. The simple branching process with infinite mean. I. J. Appl. Probability, 10:206-212, 1973.

[41] Al'bert Nikolaevich Shiryaev. Probability,, volume 95. Springer-Verlag, New York, second edition edition, 1996. 
[42] Aad W. van der Vaart. Asymptotic statistics, volume 3 of Cambridge Series in Statistical and Probabilistic Mathematics. Cambridge University Press, Cambridge, 1998.

[43] John Winnicki. Estimation theory for the branching process with immigration. In Statistical inference from stochastic processes (Ithaca, NY, 1987), volume 80 of Contemp. Math., pages 301-322. Amer. Math. Soc., Providence, RI, 1988. 